\title{
Supporting Information: Characterizing Regional Methane Emissions from Natural Gas Liquid Unloading
}

George G. Zaimes ${ }^{\mathrm{a}}$, James A. Littlefield ${ }^{\mathrm{a}, *}$, Daniel J. Augustine ${ }^{\mathrm{a}}$, Gregory Cooney ${ }^{\mathrm{a}}$, Stefan Schwietzke $^{\mathrm{b}, \mathrm{c}, \mathrm{d},+}$, Fiji C. George ${ }^{\mathrm{e}}$, Terri Lauderdale ${ }^{\mathrm{f}, \neq}, \&$ Timothy J. Skone ${ }^{\mathrm{a}}$

\author{
Author Affiliations \\ aNational Energy Technology Laboratory, 626 Cochrans Mill Road, P.O. Box 10940, Pittsburgh, \\ Pennsylvania 15236, United States

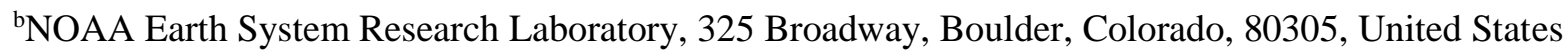 \\ ${ }^{c}$ Cooperative Institute for Research in Environmental Sciences, University of Colorado, 216 UCB, \\ Boulder, Coloardo 80309, United States

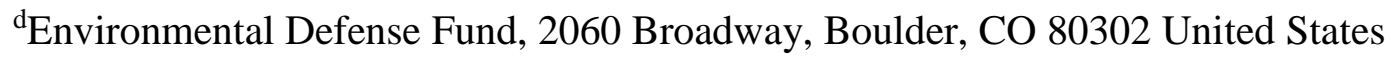 \\ ${ }^{\text {e}}$ Cheniere Energy, Inc. 700 Milam Street, Suite 1900, United States \\ fAECOM 9400 Amberglen Blvd. Austin, Texas 78729, United States
}

*Corresponding Author

Telephone: (412) 386-7560

Email: James.Littlefield@netl.doe.gov

+ Stefan Schwietzke was affiliated with NOAA Earth System Research Laboratory and Cooperative Institute for Research in Environmental Sciences during the preparation of the manuscript, and is currently employed at the Environmental Defense Fund (EDF).

${ }^{\ddagger}$ Deceased 


\section{Table of Contents}

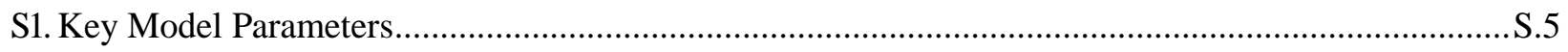

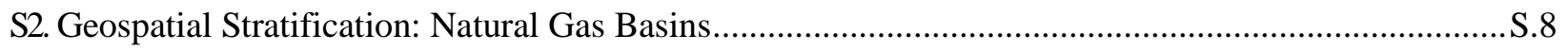

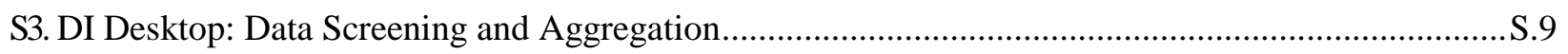

S4. Emissions Attribution: Energy-Based Allocation......................................................................... 14

S5. Probability Distribution Fitting: Venting Duration \& Venting Frequency....................................... S.14

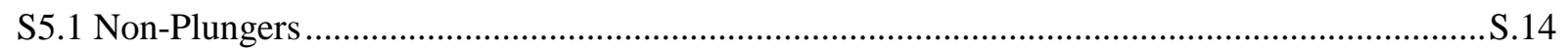

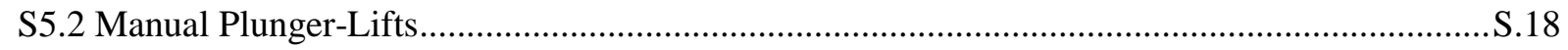

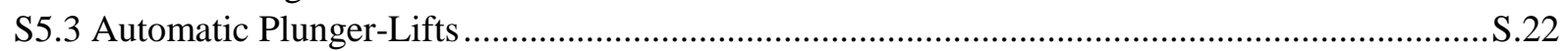

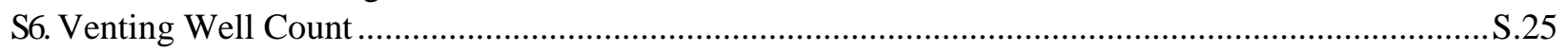

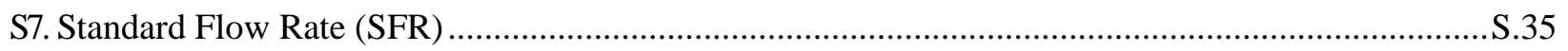

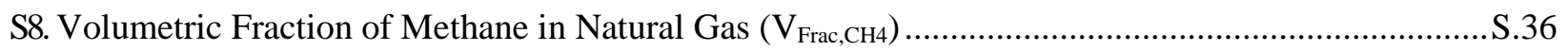

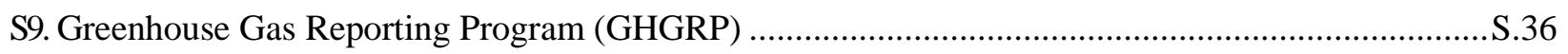

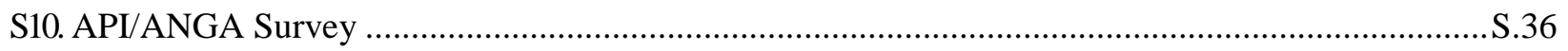

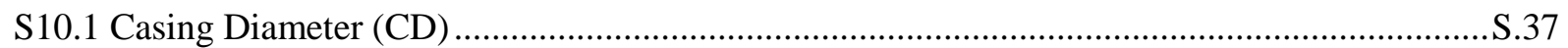

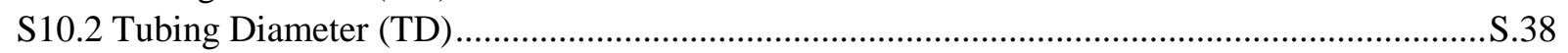

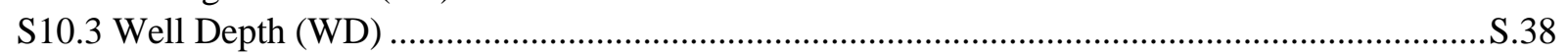

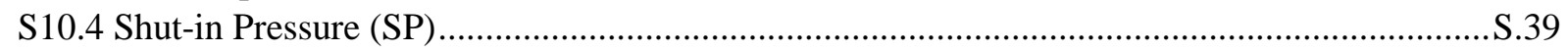

S11. Throughput Normalized Methane Emissions (TNME) ................................................................ .40

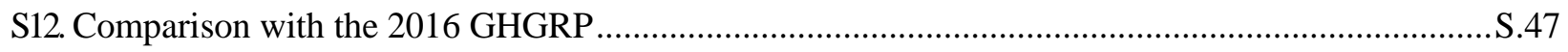

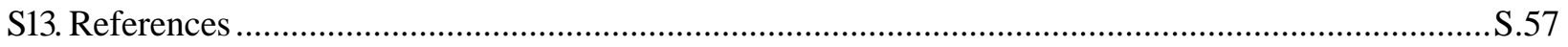




\section{Tables}

Table S1. Key parameters used to estimate methane emission from natural gas liquids unloading .........S.5

Table S2. Mapping of 18 Natural Gas Basins to NEMS regions. ............................................................

Table S3. Basin-level Well Depth Stratified by Well Type, from DI Desktop .................................... 11

Table S4. 2016 Natural gas production, concurrent oil production, and well count across unfiltered and filtered data from DI Desktop

S. 12

Table S5. Filtered DI Desktop Natural Gas Production, Oil Production, and Well Count, stratified by well

type S.13

Table S6. Non-plunger systems, venting frequency and event duration .............................................15

Table S7. Goodness of Fit Indicators, Non-Plungers, Venting Duration .............................................. 16

Table S8. Goodness of Fit Criteria, Non-Plungers, Venting Frequency ............................................... 17

Table S9. Manual plunger-lift systems, venting frequency and event duration ...................................... 18

Table S10. Goodness of Fit Criteria, Manual Plunger-Lifts, Venting Duration ....................................... 19

Table S11. Goodness of Fit Criteria, Manual Plunger-Lifts, Venting Frequency ................................. S.20

Table S12. Automatic plunger-lift systems, venting frequency and event duration .............................S.22

Table S13. Goodness of Fit Criteria, Automatic Plunger-Lifts, Venting Duration.................................2.23

Table S14. Goodness of Fit Criteria, Automatic Plunger-Lifts, Venting Frequency ............................S.24

Table S15. Basin-level well count ( $\left.\mathrm{W}_{\text {GHGRP,Basin }}\right)$ from the 2016 GHGRP ...........................................2.27

Table S16. Basin-level venting well count $\left(\mathrm{W}_{\text {vent,GHGRP,Basin }}\right)$ inferred from the 2016 GHGRP...............S.31

Table S17. Basin-level Venting Well Fractions $\left(\mathrm{F}_{\text {vent,Basin }}\right)$............................................................... 32

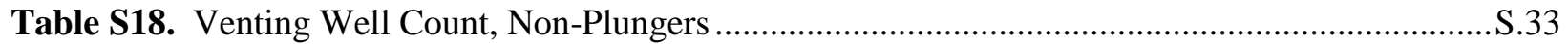

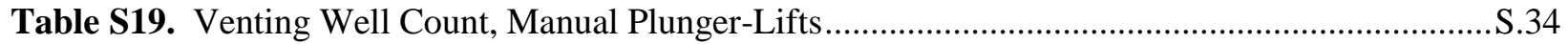

Table S20. Venting Well Count, Automatic Plunger-Lifts ......................................................................3.

Table S21. Volumetric Fraction of Methane in Natural Gas $\left(\mathrm{V}_{\mathrm{Frac}, \mathrm{CH} 4}\right)$ by NEMS Region.................... S.36

Table S22. Well parameters for conventional gas wells without plunger lifts ......................................37

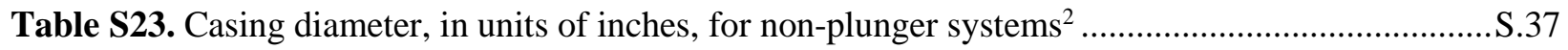

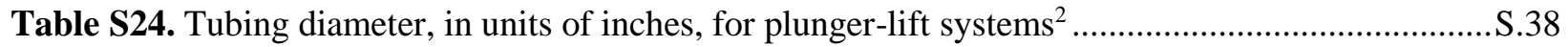

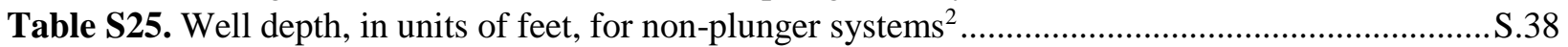

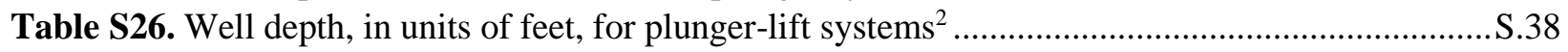

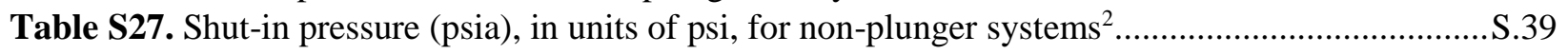

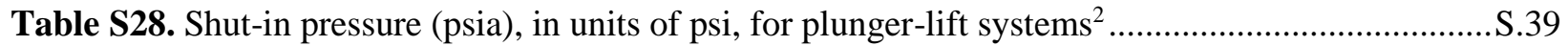

Table S29. TNME from Natural Gas Liquids Unloading, and comparison with a composite TNME $_{\text {GHGRP }}$

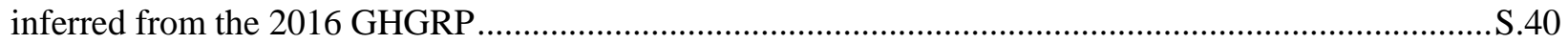

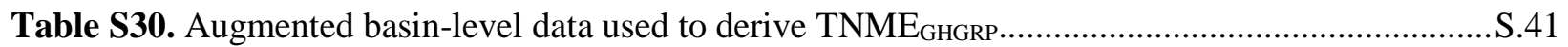

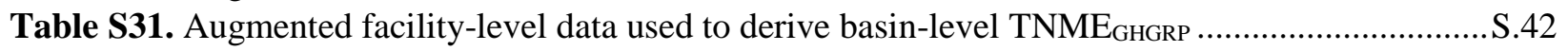

Table S32. Benchmarking liquids unloading activities in the Anadarko basin, comparison between the

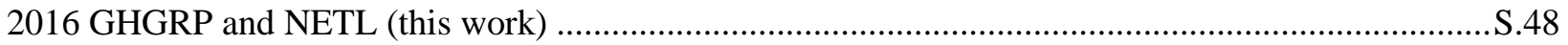

Table S33. Benchmarking liquids unloading activities in the Appalachian Eastern Overthrust basin, comparison between the 2016 GHGRP and NETL (this work) ..........................................................48

Table S34. Benchmarking liquids unloading activities in the Appalachian basin, comparison between the

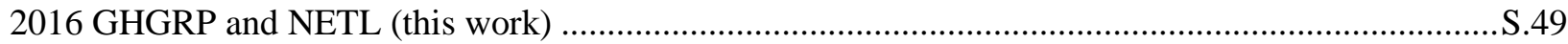

Table S35. Benchmarking liquids unloading activities in the Arkla basin, comparison between the 2016

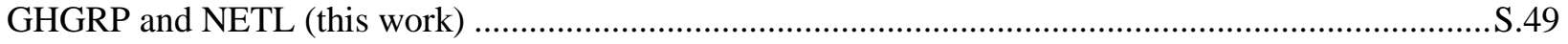

Table S36. Benchmarking liquids unloading activities in the Arkoma basin, comparison between the

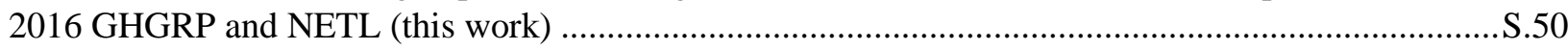


Table S37. Benchmarking liquids unloading activities in the Chautauqua basin, comparison between the 2016 GHGRP and NETL (this work)

Table S38. Benchmarking liquids unloading activities in the Denver basin, comparison between the 2016 GHGRP and NETL (this work)

Table S39. Benchmarking liquids unloading activities in the East Texas basin, comparison between the 2016 GHGRP and NETL (this work)

Table S40. Benchmarking liquids unloading activities in the Fort Worth Syncline basin, comparison between the 2016 GHGRP and NETL (this work)

S.52

Table S41. Benchmarking liquids unloading activities in the Green River basin, comparison between the 2016 GHGRP and NETL (this work)

Table S42. Benchmarking liquids unloading activities in the Gulf Coast basin, comparison between the 2016 GHGRP and NETL (this work)

Table S43. Benchmarking liquids unloading activities in the Permian basin, comparison between the 2016 GHGRP and NETL (this work)

Table S44. Benchmarking liquids unloading activities in the Piceance basin, comparison between the 2016 GHGRP and NETL (this work)

Table S45. Benchmarking liquids unloading activities in the San Juan basin, comparison between the 2016 GHGRP and NETL (this work)

Table S46. Benchmarking liquids unloading activities in the South Oklahoma Folded Belt basin, comparison between the 2016 GHGRP and NETL (this work)

Table S47. Benchmarking liquids unloading activities in the Strawn basin, comparison between the 2016 GHGRP and NETL (this work)

Table S48. Benchmarking liquids unloading activities in the Uinta basin, comparison between the 2016 GHGRP and NETL (this work)

Table S49. Benchmarking liquids unloading activities in the Williston basin, comparison between the 2016 GHGRP and NETL (this work) 


\section{Figures}

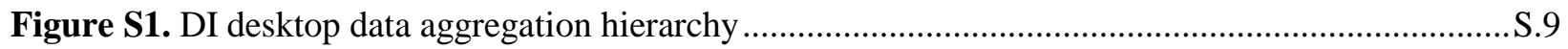

Figure S2. Detailed workflow for screening and aggregating data from DI Desktop..........................S.10

Figure S3. Simulated Liquids Unloading Venting Duration (seconds), Non-Plungers .........................S.16

Figure S4. Simulated Liquids Unloading Venting Frequency (vents/well-year), Non-Plungers .............S.17

Figure S5. Simulated Liquids Unloading Venting Duration (seconds), Manual Plunger-Lifts .............. S.20

Figure S6. Simulated Liquids Unloading Venting Frequency (vents/well-year), Manual Plunger-Lifts S.21

Figure S7. Simulated Liquids Unloading Venting Duration (seconds), Automatic Plunger-Lift ...........S.23

Figure S8. Simulated Liquids Unloading Venting Frequency (vents/well-year), Automatic Plunger-Lift

Figure S9. Wells disaggregated by well type, venting, and liquids unloading system. ........................ .25

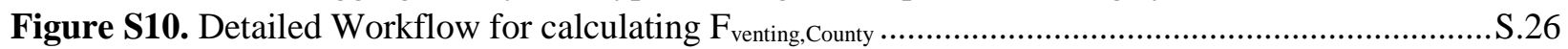

Figure S11. Augmented Data Structure GHGRP.............................................................................28 


\section{S1. Key Model Parameters}

Key parameters used in estimating methane emissions from liquids unloading include: venting frequency (V), casing diameter (CD) or tubing diameter (TD), well depth (WD), shut in pressure (SP), standard flow rate (SFR), and venting duration (HR), volumetric fraction of methane in produced natural gas $\left(\mathrm{V}_{\mathrm{Frac}, \mathrm{CH} 4}\right)$, volumetric density of methane (Density ${ }_{\mathrm{CH} 4}$ ), and fraction of methane emissions from liquids unloading that are allocated to the methane component of natural gas (Alloc $\left.\mathrm{CH}_{4}\right)$ - summarized in Table S1.

Table S1. Key parameters used to estimate methane emission from natural gas liquids unloading

\begin{tabular}{|c|c|c|c|c|c|}
\hline Parameter & Units & Data-Source & & Data Resolution & Definition \\
\hline Enon-plunger $_{\text {n }}$ & $\begin{array}{l}\mathrm{kg} \mathrm{CH}_{4} \text { year }^{-1} \\
\text { well }^{-1}\end{array}$ & Calculation & & Production Site & $\begin{array}{l}\text { Annual methane emissions from non-plunger } \\
\text { systems at standard conditions }\end{array}$ \\
\hline $\mathbf{E}_{\text {plunger }}$ & $\begin{array}{l}\mathrm{kg} \mathrm{CH}_{4} \text { year }^{-1} \\
\text { well }^{-1}\end{array}$ & Calculation & & Production Site & $\begin{array}{l}\text { Annual methane emissions from plunger-lift } \\
\text { systems at standard conditions }\end{array}$ \\
\hline $0.37 \times 10^{-3}$ & N/A & N/A & & N/A & $\begin{array}{l}\text { Conversion from psia to } \mathrm{lb} \mathrm{ft}^{-2} \\
0.37 \times 10^{-3}=[(\pi / 4) /(14.7 \mathrm{psi} / \mathrm{psia} \times 144 \\
\left.\left.\mathrm{in}^{2} / \mathrm{ft}^{2}\right)\right]\end{array}$ \\
\hline CD & in & $\begin{array}{l}\text { GHGRP }^{1} \\
\text { API/ANGA }\end{array}$ & or & $\begin{array}{l}\text { Facility-level } \\
\text { (GHGRP), or } \\
\text { NEMS Region } \\
\text { (API/ANGA) }\end{array}$ & $\begin{array}{l}\text { Well casing internal diameter. } \\
\text { GHGRP provides data for well CD at the } \\
\text { facility-level, stratified by liquids unloading } \\
\text { system (non-plungers, plunger-lifts). A } \\
\text { classification scheme was used to delineate } \\
\text { data reported by GHGRP across well types } \\
\text { (conventional, unconventional). } \\
\text { API/ANGA provides data for well CD } \\
\text { stratified by well type (conventional, } \\
\text { unconventional), liquids unloading systems } \\
\text { (non-plunger, plunger-lift), and by NEMS } \\
\text { region (Northeast, Mid-Continent, Gulf } \\
\text { Coast, and Rocky Mountain). }\end{array}$ \\
\hline \multirow[t]{2}{*}{ TD } & in & $\begin{array}{l}\text { GHGRP }^{1} \\
\text { API/ANGA }\end{array}$ & or & $\begin{array}{l}\text { Facility-Level } \\
\text { (GHGRP), or } \\
\text { NEMS Region } \\
\text { (API/ANGA) }\end{array}$ & $\begin{array}{l}\text { Well tubing internal diameter. } \\
\text { GHGRP provides data for well TD at the } \\
\text { facility-level, stratified by liquids unloading } \\
\text { system (non-plungers, plunger-lifts). A } \\
\text { classification scheme was used to delineate } \\
\text { data reported by GHGRP across well types } \\
\text { (conventional, unconventional). }\end{array}$ \\
\hline & & & & & API/ANGA provides data for well TD \\
\hline
\end{tabular}




\begin{tabular}{|c|c|c|c|c|}
\hline & & & & $\begin{array}{l}\text { stratified by well type (conventional, } \\
\text { unconventional), liquids unloading systems } \\
\text { (non-plunger, plunger-lift), and NEMS region } \\
\text { (Northeast, Mid-Continent, Gulf Coast, and } \\
\text { Rocky Mountain). }\end{array}$ \\
\hline WD & $\mathrm{ft}$ & DI Desktop & Production Site & Well depth, obtained from DI desktop. \\
\hline \multirow[t]{2}{*}{ SP } & psia & API/ANGA ${ }^{2}$ & NEMS Region & $\begin{array}{l}\text { For non-plunger systems, shut-in pressure or } \\
\text { surface pressure for wells with tubing } \\
\text { production and no packers or casing pressure. } \\
\text { For plunger-lift systems, flow-line pressure } \\
\text { for wells using engineering estimates based } \\
\text { on best available information. }\end{array}$ \\
\hline & & & & $\begin{array}{l}\text { API/ANGA provides data for SP stratified by } \\
\text { well type (conventional, unconventional), } \\
\text { liquids unloading systems (non-plunger, } \\
\text { plunger-lift), and NEMS region (Northeast, } \\
\text { Mid-Continent, Gulf Coast, and Rocky } \\
\text { Mountain). }\end{array}$ \\
\hline \multirow[t]{2}{*}{$\mathbf{V}$} & $\begin{array}{l}\text { vents } \\
\text { year }^{-1}\end{array} \quad$ well ${ }^{-1}$ & Allen et al. $2015^{3}$ & Production Site & Number of vents per well per year. \\
\hline & & & & $\begin{array}{l}\text { Allen et al. } 2015 \text { provides per-well venting } \\
\text { frequency (V) stratified by liquids unloading } \\
\text { systems (non-plunger, manual plunger-lift, } \\
\text { and automatic plunger-lift). }\end{array}$ \\
\hline \multirow[t]{2}{*}{ SFR } & $\begin{array}{l}\text { scf NG hr } \\
\text { well }^{-1}\end{array}$ & $\begin{array}{l}\text { Calculated based } \\
\text { on aggregated data }\end{array}$ & County-level & Average flow-line rate of gas per well. \\
\hline & & from DI Desktop ${ }^{4}$ & & $\begin{array}{l}\text { SFR is calculated at the county-level, derived } \\
\text { from cumulative natural gas production } \\
\text { obtained via DI desktop. SFR is stratified by } \\
\text { well type (conventional and unconventional). }\end{array}$ \\
\hline \multirow[t]{2}{*}{ HR } & $\mathrm{hr}$ & Allen et al. $2015^{3}$ & Production Site & $\begin{array}{l}\text { Hours that each well was open to the } \\
\text { atmosphere during unloading. }\end{array}$ \\
\hline & & & & $\begin{array}{l}\text { Venting duration (HR) stratified by liquids } \\
\text { unloading systems (non-plunger, manual } \\
\text { plunger-lift, and automatic plunger-lift) was } \\
\text { sourced from Allen et al. } 2015 \text {. }\end{array}$ \\
\hline 1.0 & $\mathrm{hr}$ & N/A & N/A & $\begin{array}{l}\text { Hours for average well to blow-down casing } \\
\text { volume at shut-in pressure (applies only to } \\
\text { non-plunger systems). }\end{array}$ \\
\hline
\end{tabular}




\begin{tabular}{|c|c|c|c|c|}
\hline 0.5 & $\mathrm{hr}$ & N/A & N/A & $\begin{array}{l}\text { Hours for average well to blow-down tubing } \\
\text { volume at flow-line pressure (applies only to } \\
\text { plunger-lift systems). }\end{array}$ \\
\hline \multirow[t]{2}{*}{$\mathbf{Z}$} & N/A & N/A & N/A & $\begin{array}{l}\text { For non-plunger systems, if } \mathrm{HR} \text { is less than } \\
1.0 \text {, then } \mathrm{Z} \text { is equal to } 0 \text {. If } \mathrm{HR} \text { is greater than } \\
\text { or equal to } 1.0 \text {, then } \mathrm{Z} \text { is equal to } 1 \text {. }\end{array}$ \\
\hline & & & & $\begin{array}{l}\text { For plunger-lift systems, if } \mathrm{HR} \text { is less than } \\
0.5 \text {, then } \mathrm{Z} \text { is equal to } 0 \text {. If } \mathrm{HR} \text { is greater than } \\
\text { or equal to } 0.5 \text {, then } \mathrm{Z} \text { is equal to } 1 \text {. }\end{array}$ \\
\hline Densitych4 & $\begin{array}{l}\mathrm{kg} \mathrm{CH}_{4} \mathrm{scf}^{-1} \\
\mathrm{CH}_{4}^{-1}\end{array}$ & $\begin{array}{l}\text { National Institute } \\
\text { of Standards and } \\
\text { Technology } \\
(\text { NIST) })^{5}\end{array}$ & Global & $\begin{array}{l}\text { Volumetric mass density of } \mathrm{CH}_{4} \text { at standard } \\
\text { conditions }\left(0^{\circ} \mathrm{C}, 1 \mathrm{~atm}\right) \text {, equal to } 0.0203 \mathrm{~kg} \\
\mathrm{CH}_{4} \mathrm{scf}^{-1} \mathrm{CH}_{4}^{-1} \text {. }\end{array}$ \\
\hline \multirow[t]{2}{*}{ V Frac,CH4 $_{\text {C }}$} & $\begin{array}{l}\text { scf } \mathrm{CH}_{4} \mathrm{scf}^{-1} \\
\mathrm{NG}^{-1}\end{array}$ & Allen et al. $2015^{3}$ & NEMS Region & $\begin{array}{l}\text { Volumetric fraction of methane in natural } \\
\text { gas. }\end{array}$ \\
\hline & & & & $\begin{array}{l}\text { Allen et al } 2015 \text { provides } V_{\text {Frac,CH4 }} \text { stratified } \\
\text { by NEMS Region (Northeast, Mid-Continent, } \\
\text { Gulf Coast, and Rocky Mountain). }\end{array}$ \\
\hline Allocch4 & $\%$ & Calculation & $\begin{array}{l}\text { County-level } \\
\text { (NETL) } \\
\text { facility-level } \\
\text { (GHGRP) }\end{array}$ & $\begin{array}{l}\text { Alloc } \mathrm{CH}_{4} \text { represents the fraction of emissions } \\
\text { from liquids unloading that are apportioned to } \\
\text { the methane component of produced natural } \\
\text { gas. In this work, emissions from liquids } \\
\text { unloading were allocated between the } \\
\text { methane component of produced natural gas } \\
\left(\mathrm{CH}_{4}\right) \text {, natural gas liquids and condensates } \\
\left(\mathrm{C}_{2+}\right) \text {, and coproduced oil using energy-based } \\
\text { allocation. In calculation of TNNE, allocation } \\
\text { factors are derived at the county-level. } \\
\text { However, when calculating TNME } \\
\text { allocation factors are derived at the facility- } \\
\text { level. }\end{array}$ \\
\hline
\end{tabular}




\section{S2. Geospatial Stratification: Natural Gas Basins}

National gas basins are classified using the geospatial classification (Rocky Mountain, Mid-Continent, Appalachian, or Gulf Coast) defined in Allen et al. 2015², and cross-walked to National Energy Modelling System (NEMS) regions, shown in Table S2.

Table S2. Mapping of 18 Natural Gas Basins to NEMS regions.

\begin{tabular}{|c|c|c|}
\hline AAPG Basin & $\begin{array}{l}\text { Classification \& Syntax } \\
\text { (NEMS Region) }\end{array}$ & $\begin{array}{l}\text { Classification \& Syntax } \\
\text { (Allen et al. 2015) }\end{array}$ \\
\hline 360 - Anadarko Basin & Mid-Continent & Mid-Continent (MC) \\
\hline 160A - Appalachian Eastern Overthrust & Northeast & Appalachian (AP) \\
\hline 160 - Appalachian Basin & Northeast & Appalachian (AP) \\
\hline 230 - Arkla Basin & Gulf Coast & Gulf Coast (GC) \\
\hline 345 - Arkoma Basin & Mid-Continent & Mid-Continent (MC) \\
\hline 540 - Denver Basin & Rocky Mountain & Rocky Mountain (RM) \\
\hline 260 - East Texas Basin & Gulf Coast & Gulf Coast (GC) \\
\hline 420 - Fort Worth Syncline & Mid-Continent & Mid-Continent (MC) \\
\hline 535 - Green River Basin & Rocky Mountain & Rocky Mountain (RM) \\
\hline 220 - Gulf Coast Basin & Gulf Coast & Gulf Coast (GC) \\
\hline 430 - Permian Basin & Mid-Continent & Mid-Continent (MC) \\
\hline 595 - Piceance Basin & Rocky Mountain & Rocky Mountain (RM) \\
\hline 580 - San Juan Basin & Rocky Mountain & Rocky Mountain (RM) \\
\hline 415 - Strawn Basin & Mid-Continent & Mid-Continent (MC) \\
\hline 575 - Uinta Basin & Rocky Mountain & Rocky Mountain (RM) \\
\hline 395 - Williston Basin & Rocky Mountain & Rocky Mountain (RM) \\
\hline 350 - South Oklahoma Folded Belt & Mid-Continent & Mid-Continent (MC) \\
\hline 355 - Chautauqua Platform & Mid-Continent & Mid-Continent (MC) \\
\hline
\end{tabular}




\section{S3. DI Desktop: Data Screening and Aggregation}

Key parameters for 2016 natural gas activities was constructed via screening and aggregating data from DrillingInfo (DI) desktop ${ }^{4}$. DI Desktop provides data for 2016 natural gas operations, including cumulative gas produced, cumulative oil produced, well count, drilling type, well depth, and date of first production. In this work, well-level data obtained from DI desktop is aggregated across multiple spatial scales, as shown in Figure S1. A detailed workflow for screening and aggregating data from DI desktop is provided in Figure S2. Basin-level minimum, maximum, and expected well depths, stratified by well type is provided in Table $\mathbf{S 3}$.

Figure S1. DI desktop data aggregation hierarchy

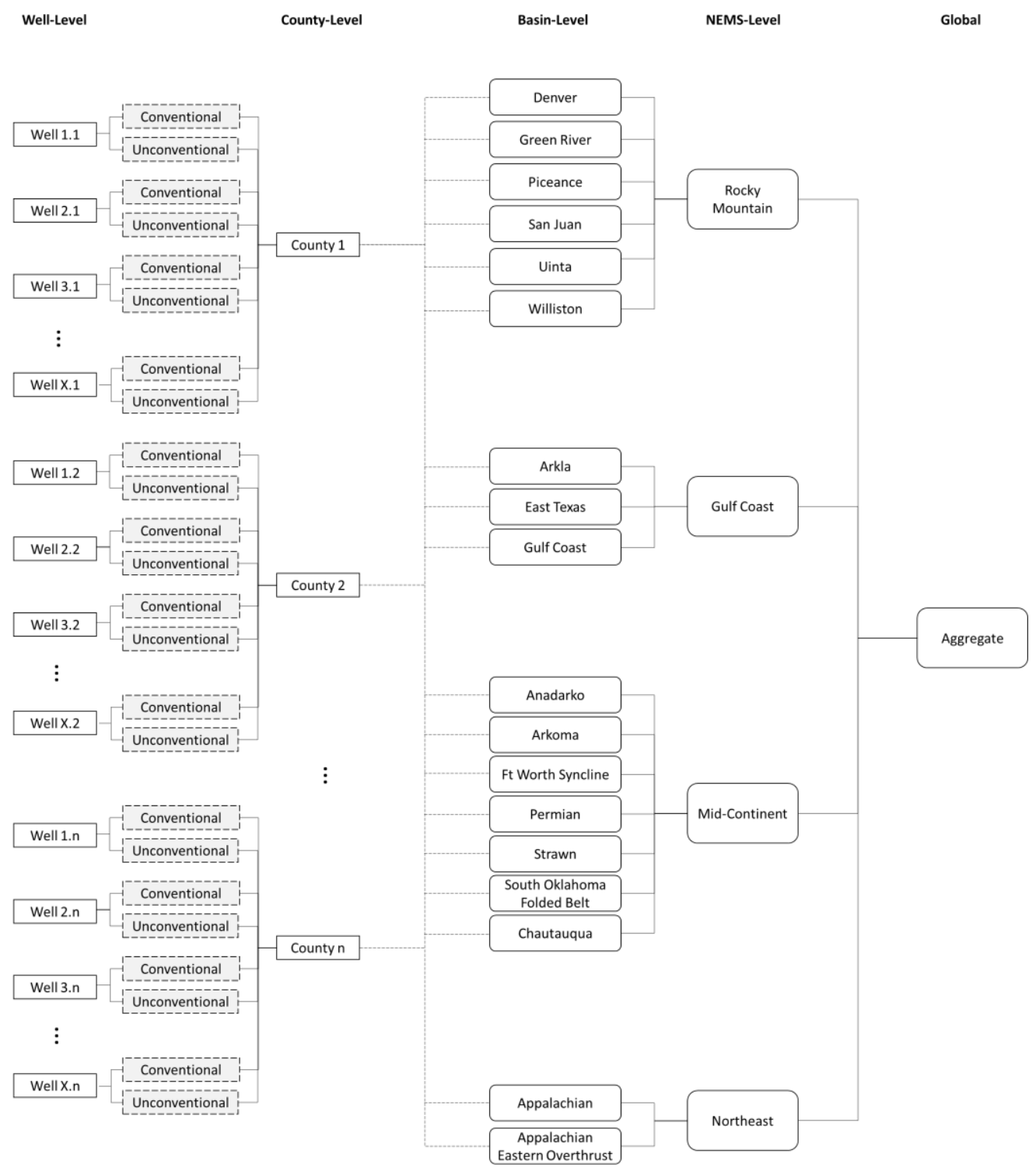


Figure S2. Detailed workflow for screening and aggregating data from DI Desktop

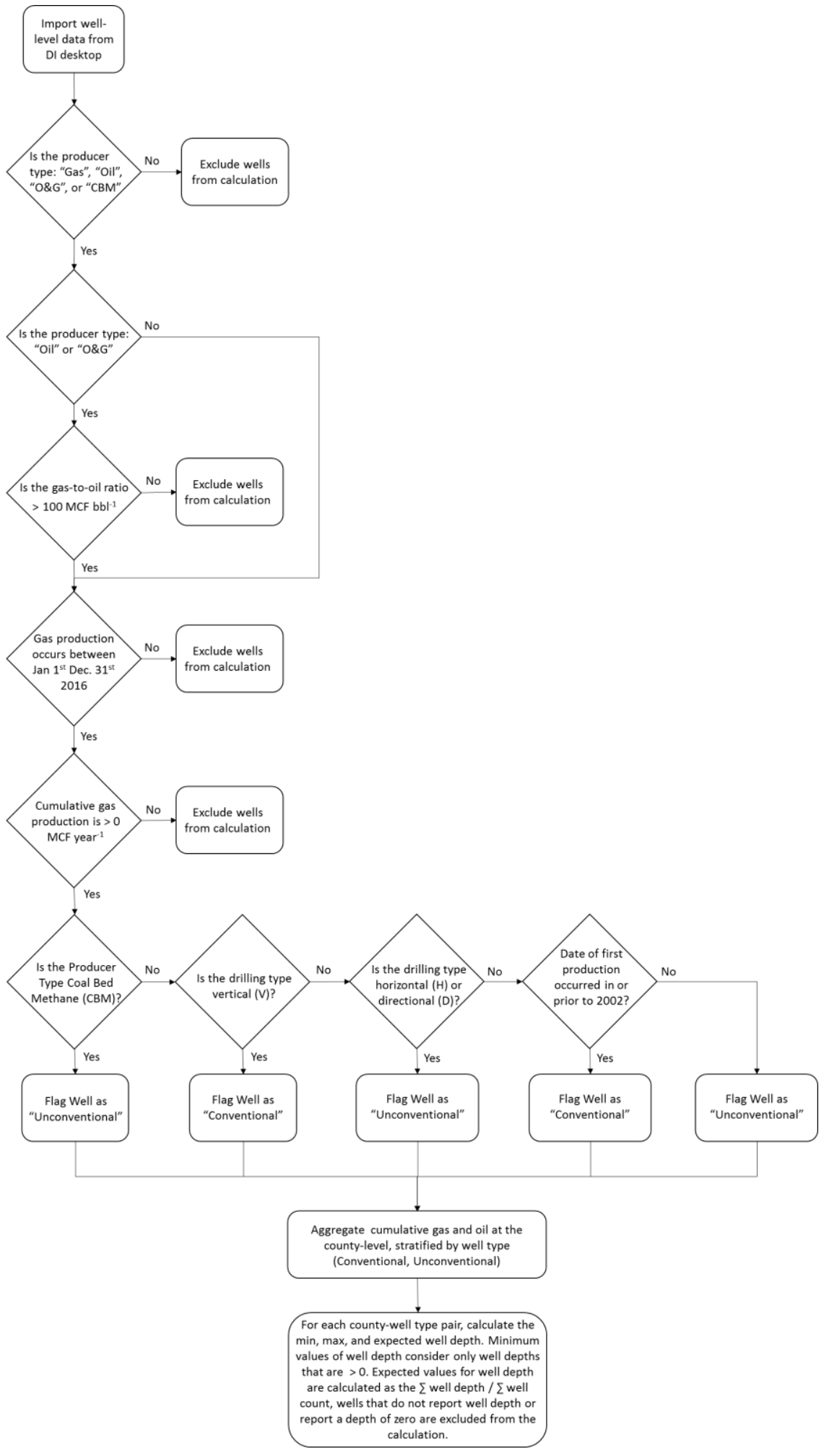


Table S3. Basin-level Well Depth Stratified by Well Type, from DI Desktop

\begin{tabular}{|c|c|c|c|c|c|c|}
\hline Basins & $\begin{array}{l}\text { Conv. } \\
\text { Minimum }\end{array}$ & $\begin{array}{l}\text { Conv. } \\
\text { Expected }\end{array}$ & $\begin{array}{l}\text { Conv. } \\
\text { Maximum }\end{array}$ & $\begin{array}{l}\text { Unconv. } \\
\text { Minimum }\end{array}$ & $\begin{array}{l}\text { Unconv. } \\
\text { Expected }\end{array}$ & $\begin{array}{l}\text { Unconv. } \\
\text { Maximum }\end{array}$ \\
\hline Anadarko Basin & 112 & 7,129 & 26,402 & 876 & 11,645 & 26,318 \\
\hline Appalachian Basin Eastern Overthrust & 40 & 3,901 & 25,470 & 14 & 7,947 & 24,578 \\
\hline Appalachian Basin & 35 & 3,975 & 18,057 & 850 & 6,830 & 19,915 \\
\hline Arkla Basin & 374 & 5,547 & 20,395 & 2,023 & 15,807 & 24,732 \\
\hline Arkoma Basin & 83 & 6,192 & 17,255 & 293 & 8,066 & 21,906 \\
\hline Chautauqua Platform & 400 & 3,706 & 13,100 & 420 & 4,271 & 18,374 \\
\hline Denver Basin & 970 & 5,975 & 10,832 & 1,565 & 6,525 & 18,760 \\
\hline East Texas Basin & 965 & 10,750 & 19,915 & 2,370 & 13,522 & 23,070 \\
\hline Fort Worth Syncline & 481 & 6,709 & 11,815 & 4,685 & 10,732 & 18,715 \\
\hline Green River Basin & 986 & 9,249 & 21,500 & 1,117 & 11,294 & 19,500 \\
\hline Gulf Coast Basin & 400 & 9,610 & 29,390 & 1,879 & 14,097 & 23,500 \\
\hline Permian Basin & 99 & 7,646 & 39,589 & 264 & 12,177 & 23,006 \\
\hline Piceance Basin & 125 & 5,420 & 19,710 & 63 & 8,628 & 19,070 \\
\hline San Juan Basin & 166 & 5,918 & 13,127 & 9 & 3,830 & 17,945 \\
\hline South Oklahoma Folded Belt & 306 & 7,819 & 21,906 & 1,415 & 13,680 & 26,089 \\
\hline Strawn Basin & 838 & 5,513 & 9,619 & 2,040 & 10,486 & 17,834 \\
\hline Uinta Basin & 874 & 7,472 & 20,900 & 900 & 8,766 & 20,781 \\
\hline Williston Basin & 720 & 1,893 & 15,025 & 1,555 & 14,628 & 22,165 \\
\hline
\end{tabular}

Table S4 shows 2016 basin-total cumulative natural gas production, oil production, and well count for the unfiltered and filtered datasets obtained from DI desktop, wherein filtered data is constructed via applying the workflow detailed in Figure $\mathbf{S 2}$ to unfiltered data sourced from DI desktop. A detailed breakdown of basin-level well count ( $\mathrm{W}_{\mathrm{DI}, \mathrm{Basin}}$ ), natural gas production, and oil production disaggregated by well type (conventional, unconventional) for natural gas activities in 2016 across eighteen U.S. natural gas basins is provided in Table $\mathbf{S 5}$. 
Table S4. 2016 Natural gas production, concurrent oil production, and well count across unfiltered and filtered data from DI Desktop

\begin{tabular}{|c|c|c|c|c|c|c|}
\hline \multirow[t]{2}{*}{ Basin } & \multicolumn{2}{|c|}{$\begin{array}{c}\text { Natural Gas Production } \\
\text { (GCF) }\end{array}$} & \multicolumn{2}{|c|}{$\begin{array}{l}\text { Oil Production } \\
\text { (Mbbl) }\end{array}$} & \multicolumn{2}{|c|}{$\begin{array}{c}\text { Well Count } \\
\text { (\# Wells) }\end{array}$} \\
\hline & Unfiltered & Filtered* & Unfiltered & Filtered $*$ & Unfiltered & Filtered* \\
\hline Anadarko Basin & 2,105 & 1,565 & 133,253 & 26,809 & 77,685 & 50,309 \\
\hline Appalachian Basin Eastern Overthrust & 7,870 & 7,550 & 25,559 & 8,561 & 130,933 & 103,667 \\
\hline Appalachian Basin & 296 & 183 & 9,709 & 357 & 55,884 & 38,147 \\
\hline Arkla Basin & 1,442 & 1,426 & 17,029 & 2,088 & 33,838 & 16,346 \\
\hline Arkoma Basin & 1,255 & 1,253 & 2,616 & 132 & 20,992 & 19,127 \\
\hline Chautauqua Platform & 120 & 59 & 26,035 & 658 & 25,152 & 5,270 \\
\hline Denver Basin & 677 & 170 & 112,523 & 7,508 & 30,589 & 13,343 \\
\hline East Texas Basin & 1,245 & 1,172 & 21,433 & 4,663 & 32,074 & 21,626 \\
\hline Fort Worth Syncline & 605 & 579 & 5,148 & 2,164 & 16,681 & 11,275 \\
\hline Green River Basin & 1,309 & 1,255 & 13,892 & 9,055 & 13,581 & 12,661 \\
\hline Gulf Coast Basin & 3,215 & 2,354 & 538,783 & 105,447 & 61,760 & 24,911 \\
\hline Permian Basin & 2,628 & 706 & 747,381 & 36,814 & 156,034 & 27,543 \\
\hline Piceance Basin & 596 & 594 & 5,848 & 1,916 & 17,703 & 17,076 \\
\hline San Juan Basin & 957 & 919 & 7,381 & 1,605 & 30,372 & 28,893 \\
\hline South Oklahoma Folded Belt & 287 & 170 & 27,724 & 1,817 & 16,047 & 2,802 \\
\hline Strawn Basin & 809 & 809 & 117 & 116 & 8,081 & 8,049 \\
\hline Uinta Basin & 346 & 297 & 24,094 & 1,692 & 11,673 & 7,414 \\
\hline Williston Basin & 658 & 25 & 399,135 & 107 & 19,565 & 2,963 \\
\hline Rocky Mountain & 4,542 & 3,259 & 562,873 & 21,883 & 123,483 & 82,350 \\
\hline Gulf Coast & 5,901 & 4,951 & 577,245 & 112,197 & 127,672 & 62,883 \\
\hline Mid-Continent & 7,809 & 5,141 & 942,273 & 68,510 & 320,673 & 124,375 \\
\hline Northeast & 8,166 & 7,734 & 35,268 & 8,918 & 186,817 & 141,814 \\
\hline Aggregate & 26,419 & 21,085 & $2,117,659$ & 211,508 & 758,644 & 411,422 \\
\hline
\end{tabular}


Table S5. Filtered DI Desktop Natural Gas Production, Oil Production, and Well Count, stratified by well type

\begin{tabular}{|c|c|c|c|c|c|c|c|c|c|}
\hline \multirow[b]{2}{*}{ Basins } & \multicolumn{3}{|c|}{ Conventional } & \multicolumn{3}{|c|}{ Unconventional } & \multicolumn{3}{|c|}{ Total } \\
\hline & $\begin{array}{l}\text { Natural Gas } \\
\text { (GCF) }\end{array}$ & $\begin{array}{l}\text { Oil } \\
(\mathrm{Mbbl})\end{array}$ & $\begin{array}{l}\text { Well Count } \\
(\# \text { Wells })\end{array}$ & $\begin{array}{l}\text { Natural Gas } \\
\text { (GCF) }\end{array}$ & $\begin{array}{l}\text { Oil } \\
(\mathrm{Mbbl})\end{array}$ & $\begin{array}{l}\text { Well Count } \\
\text { (\# Wells) }\end{array}$ & $\begin{array}{l}\text { Natural Gas } \\
\text { (GCF) }\end{array}$ & $\begin{array}{l}\text { Oil } \\
(\mathrm{Mbbl})\end{array}$ & $\begin{array}{l}\text { Well Count } \\
\text { (\# Wells) }\end{array}$ \\
\hline Anadarko Basin & 680 & 4,158 & 42,614 & 885 & 22,651 & 7,695 & 1,565 & 26,809 & 50,309 \\
\hline Appalachian Basin Eastern Overthrust & 184 & 330 & 85,084 & 7,366 & 8,231 & 18,583 & 7,550 & 8,561 & 103,667 \\
\hline Appalachian Basin & 112 & 111 & 37,250 & 71 & 246 & 897 & 183 & 357 & 38,147 \\
\hline Arkla Basin & 152 & 880 & 12,559 & 1,274 & 1,208 & 3,787 & 1,426 & 2,088 & 16,346 \\
\hline Arkoma Basin & 124 & 24 & 8,006 & 1,129 & 108 & 11,121 & 1,253 & 132 & 19,127 \\
\hline Chautauqua Platform & 20 & 367 & 3,577 & 39 & 291 & 1,693 & 59 & 658 & 5,270 \\
\hline Denver Basin & 34 & 625 & 6,688 & 136 & 6,882 & 6,655 & 170 & 7,508 & 13,343 \\
\hline East Texas Basin & 475 & 2,242 & 17,057 & 696 & 2,420 & 4,569 & 1,172 & 4,663 & 21,626 \\
\hline Fort Worth Syncline & 102 & 294 & 6,149 & 477 & 1,870 & 5,126 & 579 & 2,164 & 11,275 \\
\hline Green River Basin & 358 & 865 & 3,464 & 897 & 8,189 & 9,197 & 1,255 & 9,055 & 12,661 \\
\hline Gulf Coast Basin & 521 & 7,503 & 14,181 & 1,833 & 97,944 & 10,730 & 2,354 & 105,447 & 24,911 \\
\hline Permian Basin & 342 & 2,298 & 25,075 & 364 & 34,517 & 2,468 & 706 & 36,814 & 27,543 \\
\hline Piceance Basin & 25 & 38 & 1,997 & 568 & 1,878 & 15,079 & 594 & 1,916 & 17,076 \\
\hline San Juan Basin & 335 & 1,035 & 19,600 & 584 & 570 & 9,293 & 919 & 1,605 & 28,893 \\
\hline South Oklahoma Folded Belt & 29 & 341 & 2,004 & 141 & 1,476 & 798 & 170 & 1,817 & 2,802 \\
\hline Strawn Basin & 8 & 1 & 551 & 801 & 115 & 7,498 & 809 & 116 & 8,049 \\
\hline Uinta Basin & 111 & 603 & 3,538 & 186 & 1,089 & 3,876 & 297 & 1,692 & 7,414 \\
\hline Williston Basin & 22 & 91 & 2,920 & 2 & 16 & 43 & 25 & 107 & 2,963 \\
\hline Rocky Mountain & 886 & 3,257 & 38,207 & 2,374 & 18,625 & 44,143 & 3,259 & 21,883 & 82,350 \\
\hline Gulf Coast & 1,148 & 10,625 & 43,797 & 3,803 & 101,572 & 19,086 & 4,951 & 112,197 & 62,883 \\
\hline Mid-Continent & 1,305 & 7,482 & 87,976 & 3,836 & 61,028 & 36,399 & 5,141 & 68,510 & 124,375 \\
\hline Northeast & 297 & 441 & 122,334 & 7,437 & 8,477 & 19,480 & 7,734 & 8,918 & 141,814 \\
\hline Aggregate & 3,635 & 21,806 & 292,314 & 17,450 & 189,702 & 119,108 & 21,085 & 211,508 & 411,422 \\
\hline
\end{tabular}




\section{S4. Emissions Attribution: Energy-Based Allocation}

In this work, energy-based allocation is performed to apportion methane emissions from liquids unloading across the methane component of produced natural gas $\left(\mathrm{CH}_{4}\right)$, natural gas liquids and condensates $\left(\mathrm{C}_{2+}\right)$, and co-produced oil, and is defined in Eqn (1).

$$
\operatorname{Alloc}_{C H 4}(\%)=\frac{H H V_{C H 4} \times\left(V_{N G} \times V_{F r a c, C H 4}\right)}{H H V_{N G} \times V_{N G}+H H V_{O i l} \times V_{O i l}}
$$

In energy-based allocation, emissions are weighted across products based on the energy content (e.g. BTU) of each product stream. The energy content of natural gas is calculated as the product of the volumetric energy density $\left(\mathrm{HHV}_{\mathrm{NG}}\right)$ of natural gas, $1031 \mathrm{BTU} / \mathrm{scf}-\mathrm{NG}$, and the volume of produced natural gas $\left(\mathrm{V}_{\mathrm{NG}}\right)^{6}$. The energy content of the methane component of natural gas is calculated as the product of the volumetric energy density $\left(\mathrm{HHV}_{\mathrm{NG}}\right)$ of methane, $1010 \mathrm{BTU} / \mathrm{scf}-\mathrm{CH}_{4}$, and the volume of

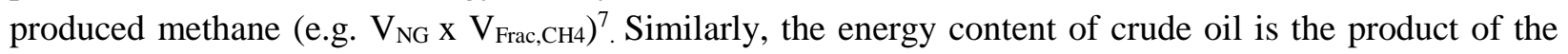
volumetric energy density $\left(\mathrm{HHV}_{\text {oil }}\right)$ of crude oil, 5.719 MMBTU per bbl-crude, and the volume of produced crude $\left(\mathrm{V}_{\text {oil }}\right)^{6}$. In this work, allocation is performed at a county level, and derived from countylevel cumulative natural gas, methane component of produced natural gas, and crude oil production. However, it is important to note that when constructing $\mathrm{TNME}_{\mathrm{GHGRP}}$, allocation factors are derived at the facility-level, and based on cumulative natural gas production (MCF) and saleable oil production (bbl) reported to the GHGRP. Additional information can be found in section S11.

\section{S5. Probability Distribution Fitting: Venting Duration \& Venting Frequency}

Data from Allen et al. $2015^{3}$ is used to parameterize the venting duration (HR) and venting frequency (V) for non-plunger, manual plunger-lift, and automatic plunger-lift systems. Multiple probability distributions are fit against the sample data including: exponential, lognormal, inverse gaussian, loglogistic, gamma, weibull, triangular, kumaraswamy, and others. Additionally, to avoid sampling a (physically impossible) negative venting frequency or venting duration, only distributions with nonnegative ranges are considered. Multiple Goodness Of Fit (GOF) indicators are used to determine the distribution that best characterizes the underlying data.

\section{S5.1 Non-Plungers}

The venting frequency and event duration for non-plunger systems, reported in Allen et al. $2015^{3}$, is provided in Table $\mathbf{S 6}$. 
Table S6. Non-plunger systems, venting frequency and event duration

\begin{tabular}{|c|c|c|c|c|c|}
\hline Well Code & $\begin{array}{l}\text { Study } \\
\text { Region }\end{array}$ & $\begin{array}{l}\text { Well } \\
\text { Direction }\end{array}$ & $\begin{array}{l}\text { Methane in } \\
\text { Produced Gas (\%) }\end{array}$ & $\begin{array}{l}\text { Average Sampled } \\
\text { Event Duration (s) }\end{array}$ & $\begin{array}{l}\text { Annual Venting Events } \\
\text { Reported by Operator }\end{array}$ \\
\hline UBB-47-0101 & GC & Vertical & 92.7 & 1,518 & 2 \\
\hline UBB-47-0201 & GC & Vertical & 80.7 & 3,417 & 4 \\
\hline UBB-47-0401 & GC & Vertical & 86.5 & 2,104 & 1 \\
\hline UCG-03-0101 & GC & Vertical & 96.1 & 6,762 & 48 \\
\hline UCG-03-0102 & GC & Vertical & 96.1 & 6,016 & 6 \\
\hline UCG-03-0103 & GC & Vertical & 96.1 & 7,919 & 12 \\
\hline UCG-03-0201 & GC & Vertical & 93.3 & 3,952 & 3 \\
\hline UCG-03-0202 & GC & Vertical & 93.3 & 4,739 & 3 \\
\hline UCG-03-0203 & GC & Vertical & 96.1 & 2,504 & 185 \\
\hline UCG-03-0204 & $\mathrm{GC}$ & Vertical & 93.3 & 5,120 & 81 \\
\hline UCG-03-0301 & GC & Vertical & 93.3 & 9,819 & 27 \\
\hline UCG-03-0302 & GC & Vertical & 96.1 & 4,308 & 151 \\
\hline UCG-03-0401 & GC & Vertical & 96.1 & 1,270 & 9 \\
\hline UCG-03-0402 & GC & Vertical & 96.1 & 2,662 & 45 \\
\hline UEY-41-0601 & $\mathrm{MC}$ & Horizontal & 95.2 & 11,782 & 1 \\
\hline UMB-06-0701 & $\mathrm{MC}$ & Vertical & 74.3 & 1,841 & 5 \\
\hline UMB-06-0801 & MC & Vertical & 72.8 & 3,580 & 3 \\
\hline UMB-06-1101 & MC & Vertical & 81.3 & 1,021 & 12 \\
\hline UMB-06-1201 & $\mathrm{MC}$ & Vertical & 78.4 & 964 & 1 \\
\hline UMD-43-0101 & AP & Horizontal & 96.9 & 2,059 & 12 \\
\hline UMD-43-0201 & $\mathrm{AP}$ & Horizontal & 97 & 2,996 & 15 \\
\hline USH-42-0501 & AP & Horizontal & 97.3 & 1,252 & 6 \\
\hline USH-45-0102 & RM & Vertical & 87.8 & 2,411 & 19 \\
\hline USH-45-0104 & RM & Vertical & 87.8 & 805 & 5 \\
\hline USH-47-0101 & $\mathrm{MC}$ & Horizontal & 95.3 & 10,009 & 4 \\
\hline USH-47-0201 & $\mathrm{MC}$ & Horizontal & 96.7 & 14,525 & 95 \\
\hline USH-47-0301 & $\mathrm{MC}$ & Horizontal & 96.7 & 16,161 & 101 \\
\hline USH-47-0401 & $\mathrm{MC}$ & Horizontal & 97.5 & 7,766 & 84 \\
\hline USH-47-0601 & $\mathrm{MC}$ & Horizontal & 94.8 & 3,971 & 70 \\
\hline USH-47-0701 & $\mathrm{MC}$ & Horizontal & 97.8 & 9,741 & 45 \\
\hline USH-47-0801 & $\mathrm{MC}$ & Horizontal & 97.9 & 5,530 & 2 \\
\hline UTG-44-0101 & AP & Vertical & 95.7 & 629 & 12 \\
\hline
\end{tabular}

Appalachian: AP; Mid-Continent: MC; Gulf Coast: GC; Rocky Mountain: RM

Table S7 compares multiple GOF indicators, obtained via fitting eight probability distributions against the venting duration for non-plunger systems reported in Table S6. In this work, the venting duration for non-plunger systems is characterized as a lognormal distribution with mean of $\sim 5,134$ seconds/event and standard deviation of $\sim 5,422$, see Figure S3. 
Table S7. Goodness of Fit Indicators, Non-Plungers, Venting Duration

\begin{tabular}{llllll}
\hline Distribution & AIC & BIC & Chi-Squared & K-S & A-D \\
\hline Exponential & 610.8 & 612.2 & 2.875 & 0.1188 & 0.6402 \\
Lognormal & 608.8 & 611.3 & 1.375 & 0.0688 & 0.1971 \\
Invgauss & 608.2 & 610.7 & 1.000 & 0.0758 & 0.2564 \\
Loglogistic & 610.9 & 613.4 & 1.000 & 0.0703 & 0.2294 \\
Gamma & 609.3 & 611.9 & 1.375 & 0.0766 & 0.2531 \\
Weibull & 610.0 & 612.5 & 1.375 & 0.0766 & 0.2531 \\
Triangular & 610.3 & 612.8 & 4.000 & 0.1841 & 1.7532 \\
Kumaraswamy & 615.4 & 619.8 & 1.375 & 0.0846 & 0.3345 \\
\hline
\end{tabular}

Figure S3. Simulated Liquids Unloading Venting Duration (seconds), Non-Plungers

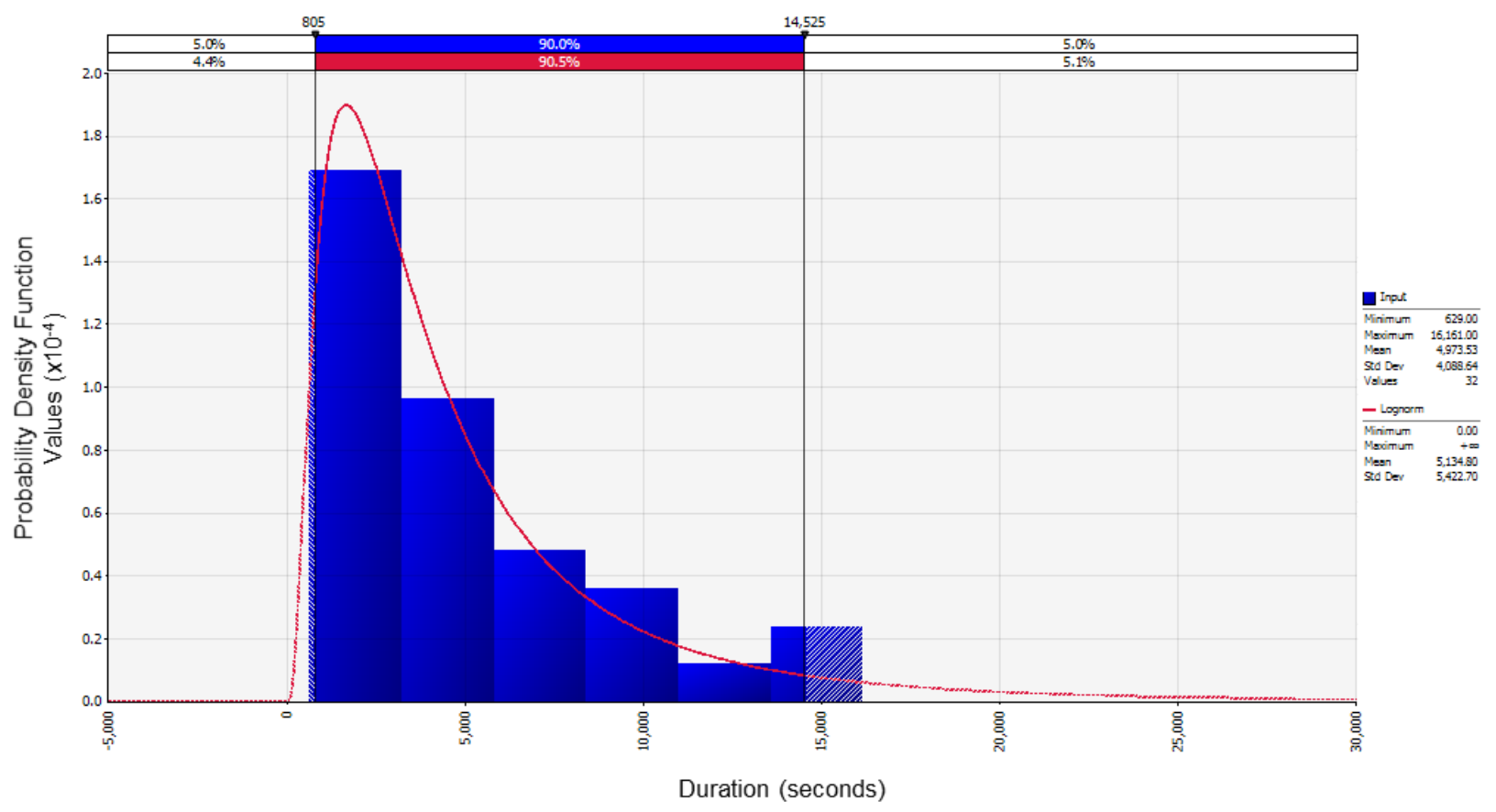

Table S8 compares multiple GOF indicators, obtained via fitting eight probability distributions against the venting frequency for non-plunger systems reported in Table S6. In work, the venting frequency of non-plunger systems is characterized as a lognormal distribution with mean of $~ 38.66$ events/well-year and standard deviation of $\sim 119.25$, see Figure S4. 
Table S8. Goodness of Fit Criteria, Non-Plungers, Venting Frequency

\begin{tabular}{llllll}
\hline Distribution & AIC & BIC & Chi-Squared & K-S & A-D \\
\hline Exponential & 290.6 & 292.0 & 21.250 & 0.2920 & 4.2027 \\
Lognormal & 281.2 & 283.7 & 7.000 & 0.1192 & 0.5052 \\
Invgauss & 279.6 & 282.1 & 6.625 & 0.1485 & 0.6797 \\
Loglogistic & 283.8 & 286.4 & 7.000 & 0.1265 & 0.5269 \\
Gamma & 286.0 & 288.6 & 6.250 & 0.1817 & 1.0358 \\
Weibull & 284.5 & 287.1 & 6.250 & 0.1505 & 0.7751 \\
Triangular & 315.31 & 317.8 & 48.625 & 0.4802 & 18.0879 \\
Kumaraswamy & N/A & N/A & N/A & N/A & N/A \\
\hline
\end{tabular}

Figure S4. Simulated Liquids Unloading Venting Frequency (vents/well-year), Non-Plungers

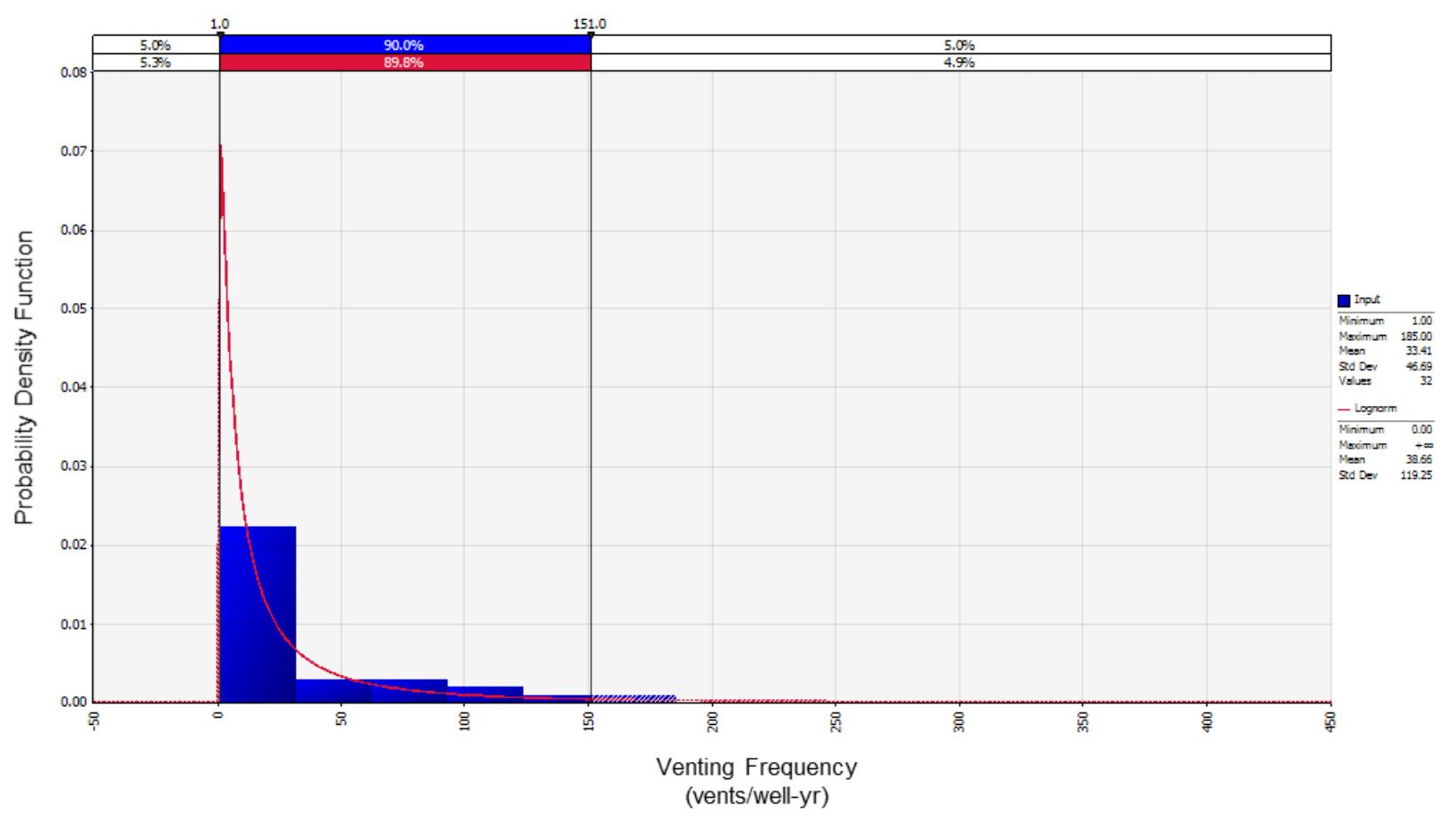




\section{S5.2 Manual Plunger-Lifts}

The venting frequency and event duration for manual plunger-lift systems, reported in Allen et al. $2015^{3}$, is provided in Table $\mathbf{S 9}$.

Table S9. Manual plunger-lift systems, venting frequency and event duration

\begin{tabular}{|c|c|c|c|c|c|}
\hline Well Code & $\begin{array}{l}\text { Study } \\
\text { Region }\end{array}$ & $\begin{array}{l}\text { Well } \\
\text { Direction }\end{array}$ & $\begin{array}{l}\text { Methane in } \\
\text { Produced Gas (\%) }\end{array}$ & $\begin{array}{l}\text { Average Sampled } \\
\text { Event Duration (s) }\end{array}$ & $\begin{array}{l}\text { Annual Venting Events } \\
\text { Reported by Operator }\end{array}$ \\
\hline UBB-45-0101 & $\mathrm{RM}$ & Vertical & 77.4 & 727 & 30 \\
\hline UBB-45-0201 & $\mathrm{RM}$ & Vertical & 77.4 & 1,529 & 50 \\
\hline UBB-45-0202 & $\mathrm{RM}$ & Vertical & 77.4 & 1,303 & 50 \\
\hline UBB-45-0203 & $\mathrm{RM}$ & Vertical & 77.4 & 1,522 & 50 \\
\hline UBB-45-0204 & RM & Vertical & 77.4 & 1,708 & 50 \\
\hline UBB-45-0301 & $\mathrm{RM}$ & Vertical & 77.4 & 1,416 & 2 \\
\hline UBB-45-0302 & $\mathrm{RM}$ & Vertical & 77.4 & 1,219 & 2 \\
\hline UBB-45-0401 & $\mathrm{RM}$ & Vertical & 77.4 & 3,714 & 2 \\
\hline UBB-45-0501 & $\mathrm{RM}$ & Vertical & 77.4 & 3,847 & 5 \\
\hline UBB-47-0301 & GC & Vertical & 86.5 & 7,672 & 2 \\
\hline UDN-44-0203 & $\mathrm{RM}$ & Vertical & 88.8 & 443 & 3 \\
\hline UDN-44-0304 & $\mathrm{RM}$ & Vertical & 90.7 & 540 & 6 \\
\hline UDN-44-0405 & $\mathrm{RM}$ & Vertical & 89.1 & 417 & 3 \\
\hline UDN-44-0506 & $\mathrm{RM}$ & Vertical & 89.9 & 528 & 1 \\
\hline UDN-44-0507 & $\mathrm{RM}$ & Vertical & 88.4 & 588 & ND \\
\hline UEF-02-0201 & $\mathrm{MC}$ & Vertical & 81.1 & 2,271 & 13 \\
\hline UEF-02-0202 & $\mathrm{MC}$ & Vertical & 84.3 & 5,637 & 10 \\
\hline UEF-02-0203 & $\mathrm{MC}$ & Vertical & 82.5 & 2,864 & 12 \\
\hline UEF-02-0204 & $\mathrm{MC}$ & Vertical & 78.4 & 1,780 & 8 \\
\hline UEF-02-0205 & $\mathrm{MC}$ & Vertical & 81.9 & 3,458 & 8 \\
\hline UEF-02-0206 & $\mathrm{MC}$ & Vertical & 85.6 & 4,996 & 4 \\
\hline UJR-46-0101 & $\mathrm{RM}$ & Vertical & 86.4 & 1,011 & 5 \\
\hline UJR-46-0401 & $\mathrm{RM}$ & Vertical & 86.4 & 231 & 6 \\
\hline UJR-46-0501 & $\mathrm{RM}$ & Vertical & 86.4 & 560 & 16 \\
\hline UJR-46-0601 & $\mathrm{RM}$ & Vertical & 86.4 & 198 & 1 \\
\hline UJR-46-0701 & $\mathrm{RM}$ & Vertical & 86.4 & 339 & 11 \\
\hline UJR-46-0801 & $\mathrm{RM}$ & Vertical & 86.4 & 3,926 & 3 \\
\hline UJR-46-1001 & $\mathrm{RM}$ & Vertical & 86.4 & 3,149 & 2 \\
\hline UJR-46-1101 & $\mathrm{RM}$ & Vertical & 86.4 & 559 & 15 \\
\hline UJR-46-1201 & $\mathrm{RM}$ & Vertical & 86.4 & 1,289 & 8 \\
\hline UMB-06-0101 & $\mathrm{MC}$ & Vertical & 83.00 & 790 & 2 \\
\hline UMB-06-0201 & $\mathrm{MC}$ & Vertical & 81.9 & 131 & 10 \\
\hline UMB-06-0301 & $\mathrm{MC}$ & Vertical & 81.8 & 2,183 & 2 \\
\hline UMB-06-0401 & $\mathrm{MC}$ & Vertical & 81.5 & 169 & 1 \\
\hline UMB-06-0501 & $\mathrm{MC}$ & Vertical & 82.1 & 474 & 2 \\
\hline UMB-06-0601 & $\mathrm{MC}$ & Vertical & 82.5 & 387 & 10 \\
\hline UMB-06-0901 & $\mathrm{MC}$ & Vertical & 77.5 & 688 & 10 \\
\hline USH-42-0301 & $\mathrm{AP}$ & Horizontal & 97.3 & 86 & 7 \\
\hline USH-45-0101 & $\mathrm{RM}$ & Vertical & 87.8 & 1,314 & 2 \\
\hline
\end{tabular}




\begin{tabular}{llllll}
\hline USH-45-0103 & RM & Vertical & 87.8 & 1,181 & 3 \\
USH-45-0105 & RM & Vertical & 87.8 & 2,997 & 15 \\
USH-45-0201 & RM & Vertical & 87.8 & 1,900 & 1 \\
USH-45-0202 & RM & Vertical & 87.8 & 1,447 & 11 \\
USH-45-0203 & RM & Vertical & 87.8 & 1,638 & 39 \\
UTG-44-0201 & AP & Vertical & 95.7 & 10,214 & 20 \\
UTG-44-0301 & AP & Vertical & 95.7 & 2,609 & 24 \\
UTG-44-0401 & AP & Vertical & 95.7 & 3,213 & 24 \\
UTG-44-0501 & AP & Vertical & 95.7 & 1,240 & 24 \\
UTG-44-0601 & AP & Vertical & 95.7 & 1,060 & 52 \\
UTG-44-0701 & AP & Vertical & 95.7 & 9,807 & 12 \\
\hline
\end{tabular}

Appalachian: AP; Mid-Continent: MC; Gulf Coast: GC; Rocky Mountain: RM

Table S10 compares multiple GOF indicators obtained via fitting eight probability distributions against the venting duration for manual plunger-lift systems reported in Table S9. In this work, the venting duration for manual plunger-lift systems is characterized as a lognormal distribution with mean of $\sim 2,194$ seconds/event and standard deviation of $\sim 3,292$, see Figure S5.

Table S10. Goodness of Fit Criteria, Manual Plunger-Lifts, Venting Duration

\begin{tabular}{llllll}
\hline Distribution & AIC & BIC & Chi-Squared & K-S & A-D \\
\hline Exponential & 865.0 & 866.9 & 7.92 & 0.0813 & 0.3907 \\
Lognormal & 864.8 & 868.3 & 8.88 & 0.0889 & 0.2284 \\
Invgauss & 868.1 & 871.7 & 5.36 & 0.1475 & 0.8439 \\
Loglogistic & 866.2 & 869.8 & 6.64 & 0.0751 & 0.2421 \\
Gamma & 867.0 & 870.6 & 6.96 & 0.0892 & 0.4260 \\
Weibull & 867.2 & 870.8 & 7.92 & 0.0834 & 0.3961 \\
Triangular & 891.9 & 895.5 & 31.92 & 0.3595 & 13.0292 \\
Kumaraswamy & N/A & N/A & N/A & N/A & N/A \\
\hline
\end{tabular}


Figure S5. Simulated Liquids Unloading Venting Duration (seconds), Manual Plunger-Lifts

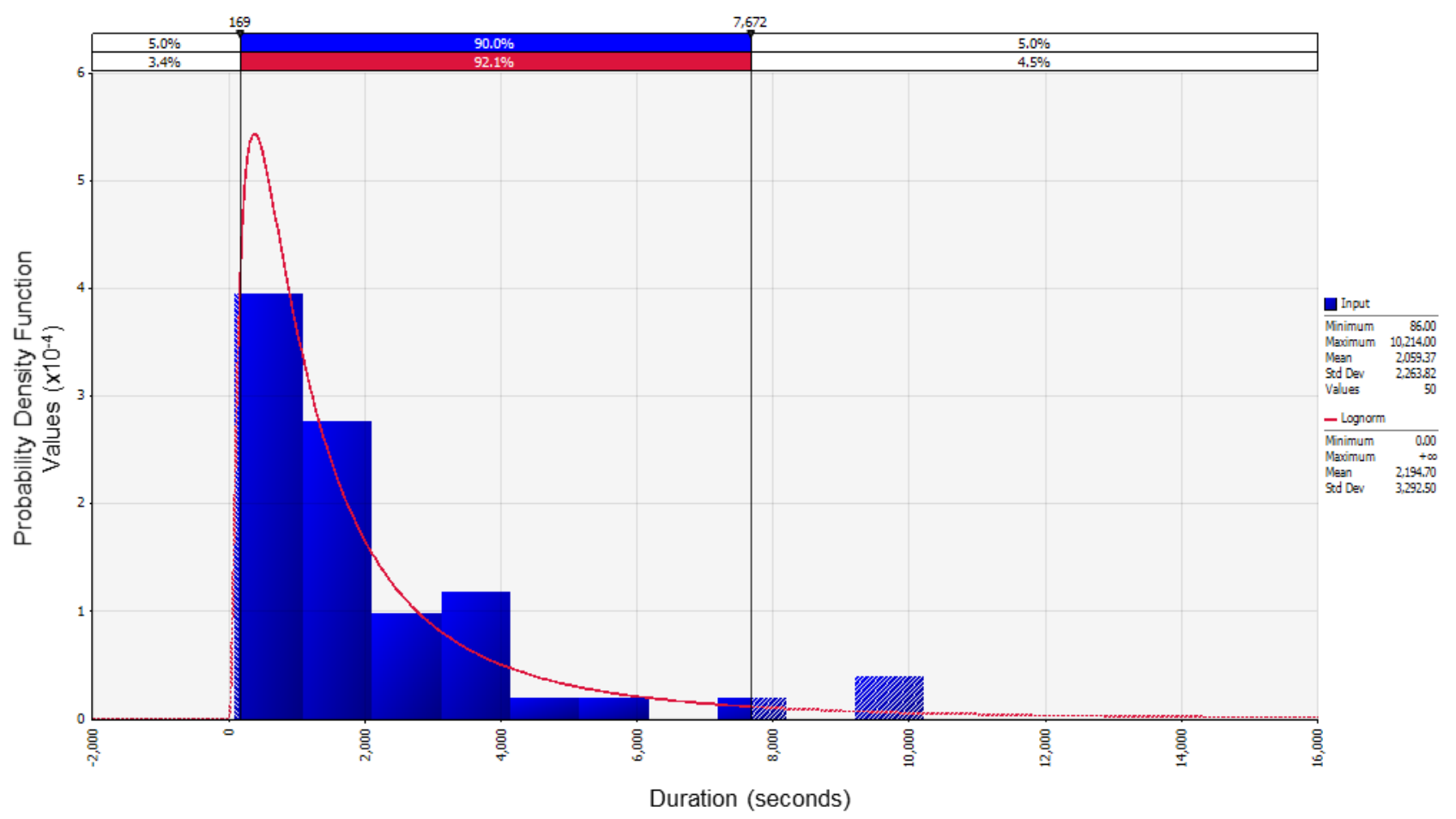

Table S11 compares multiple GOF indicators, obtained via fitting eight probability distributions against the venting frequency for manual plunger-lift systems reported in Table S9. In this work, the venting frequency for manual plunger-lift systems is characterized as a lognormal distribution with mean of $\sim 13.99$ events/well-year and standard deviation of $~ 23.84$, see Figure S6.

Table S11. Goodness of Fit Criteria, Manual Plunger-Lifts, Venting Frequency

\begin{tabular}{llllll}
\hline Distribution & AIC & BIC & Chi-Squared & K-S & A-D \\
\hline Exponential & 353.2 & 355.0 & 11.5714 & 0.1443 & 1.3281 \\
Lognormal & 350.2 & 353.8 & 13.5306 & 0.1261 & 0.7094 \\
Invgauss & 348.0 & 351.5 & 5.3673 & 0.1198 & 0.8408 \\
Loglogistic & 354.0 & 357.5 & 13.5306 & 0.1238 & 0.7393 \\
Gamma & 355.2 & 358.8 & 12.2245 & 0.1283 & 1.1068 \\
Weibull & 354.8 & 358.3 & 12.2245 & 0.1145 & 0.9328 \\
Triangular & 369.4 & 373.0 & 35.0816 & 0.3284 & 11.6445 \\
Kumaraswamy & 360.7 & 367.4 & 13.8571 & 0.1138 & 1.2227 \\
\hline
\end{tabular}


Figure S6. Simulated Liquids Unloading Venting Frequency (vents/well-year), Manual Plunger-Lifts

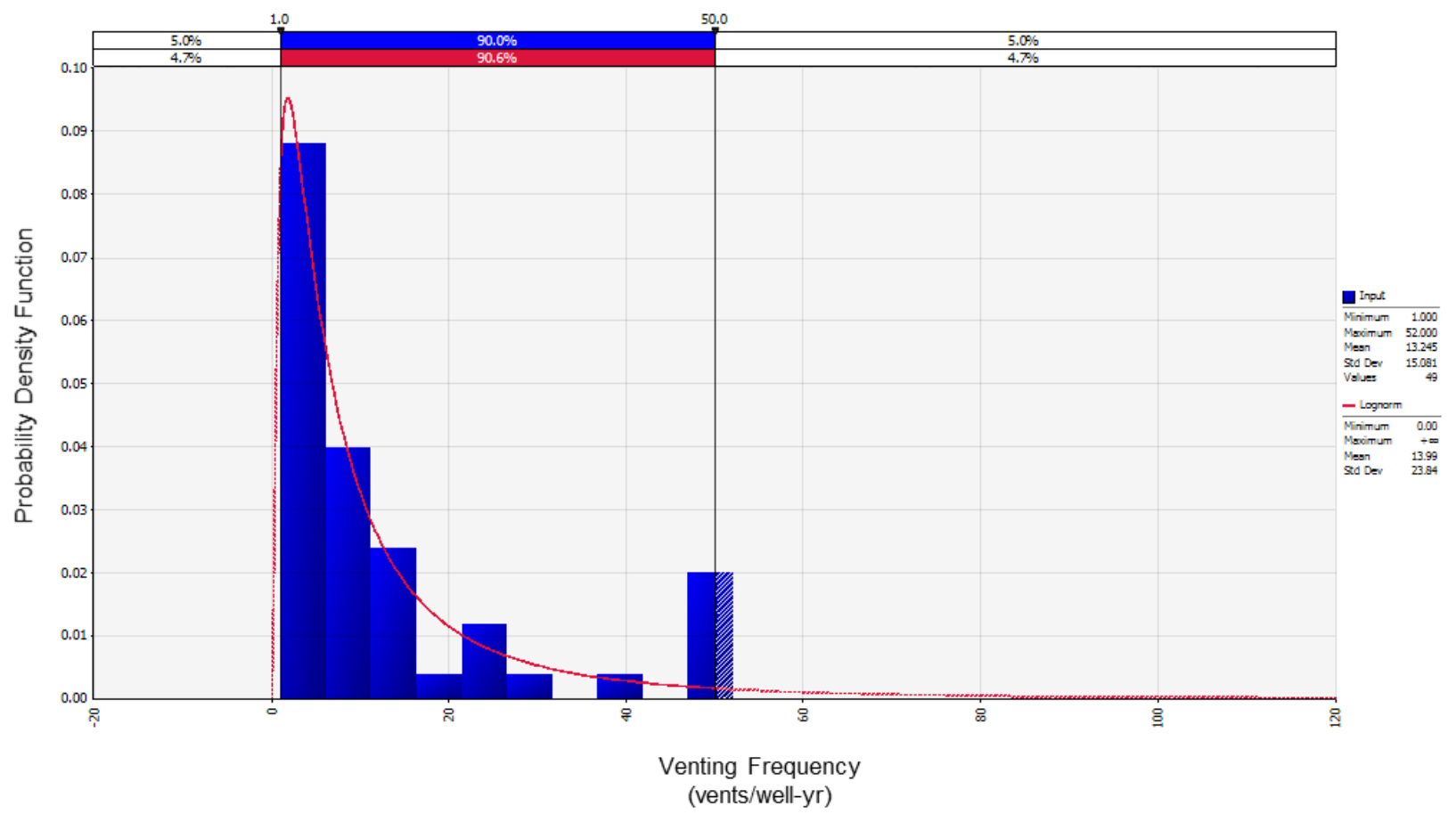




\section{S5.3 Automatic Plunger-Lifts}

The venting frequency and event duration for automatic plunger-lift systems, reported in Allen et al. 2015 ${ }^{3}$, is provided in Table $\mathbf{S 1 2}$.

Table S12. Automatic plunger-lift systems, venting frequency and event duration

\begin{tabular}{|c|c|c|c|c|c|}
\hline Well Code & $\begin{array}{l}\text { Study } \\
\text { Region }\end{array}$ & $\begin{array}{l}\text { Well } \\
\text { Direction }\end{array}$ & $\begin{array}{l}\text { Methane in } \\
\text { Produced Gas (\%) }\end{array}$ & $\begin{array}{l}\text { Average Sampled } \\
\text { Event Duration (s) }\end{array}$ & $\begin{array}{l}\text { Annual Venting Events } \\
\text { Reported by Operator }\end{array}$ \\
\hline UBB-42-0101 & $\mathrm{RM}$ & Vertical & 75.7 & 147 & 1982 \\
\hline UBB-42-0201 & RM & Vertical & 78.7 & 1208 & 1069 \\
\hline UBB-42-0401 & $\mathrm{RM}$ & Vertical & 81.9 & 389 & 2546 \\
\hline UBB-42-0501 & $\mathrm{RM}$ & Vertical & 82.9 & 130 & 606 \\
\hline UBB-42-0601 & $\mathrm{RM}$ & Vertical & 83.4 & 1208 & 2686 \\
\hline UBB-42-0701 & $\mathrm{RM}$ & Vertical & 81.1 & 276 & 184 \\
\hline UBB-42-0801 & RM & Vertical & 80.5 & 433 & 2048 \\
\hline UBB-42-0901 & RM & Vertical & 91.5 & 70 & 964 \\
\hline UBB-42-1001 & $\mathrm{RM}$ & Vertical & 91.5 & 36 & 715 \\
\hline UBB-42-1101 & RM & Vertical & 80 & 262 & 1011 \\
\hline UBB-42-1201 & $\mathrm{RM}$ & Vertical & 80.4 & 617 & 573 \\
\hline UBB-43-0101 & $\mathrm{RM}$ & Vertical & 78.5 & 177 & 2873 \\
\hline UBB-43-0301 & $\mathrm{RM}$ & Vertical & 81.7 & 137 & 4698 \\
\hline UBB-50-2601 & $\mathrm{RM}$ & Vertical & 76.8 & 317 & 643 \\
\hline UBB-50-2701 & RM & Vertical & 77.7 & 849 & 4252 \\
\hline UBB-50-2801 & $\mathrm{RM}$ & Vertical & 78.2 & 33 & 4051 \\
\hline UBB-50-2901 & $\mathrm{RM}$ & Vertical & 83 & 90 & 2482 \\
\hline UBB-50-3001 & RM & Vertical & 81.9 & 692 & 650 \\
\hline UBB-50-3101 & $\mathrm{RM}$ & Vertical & 80.6 & 73 & 1963 \\
\hline UBB-50-3201 & $\mathrm{RM}$ & Vertical & 76.6 & 309 & 528 \\
\hline UEF-49-0501 & GC & Vertical & 82.4 & 123 & 4238 \\
\hline UEY-41-0101 & $\mathrm{MC}$ & Vertical & 97.8 & 400 & 6570 \\
\hline UEY-41-0201 & MC & Vertical & 97.8 & 274 & 2389 \\
\hline UEY-41-0301 & $\mathrm{MC}$ & Vertical & 97.8 & 191 & 7509 \\
\hline UEY-41-0401 & $\mathrm{MC}$ & Vertical & 97.8 & 206 & 3893 \\
\hline
\end{tabular}

Appalachian: AP; Mid-Continent: MC; Gulf Coast: GC; Rocky Mountain: RM

Table S13 compares multiple GOF indicators, obtained via fitting eight probability distributions against the venting duration for automatic plunger-lift systems reported in Table S12. In this work, the venting duration for automatic plunger-lift systems is characterized as a lognormal distribution with mean of $\sim 358$ seconds/event and standard deviation of $\sim 437$, see Figure S7. 
Table S13. Goodness of Fit Criteria, Automatic Plunger-Lifts, Venting Duration

\begin{tabular}{llllll}
\hline Distribution & AIC & BIC & Chi-Squared & K-S & A-D \\
\hline Exponential & 344.4 & 345.5 & 1.20 & 0.1032 & 0.3773 \\
Lognormal & 344.5 & 346.4 & 0.00 & 0.0787 & 0.1537 \\
Invgauss & 344.8 & 346.7 & 1.20 & 0.1104 & 0.2905 \\
Loglogistic & 345.4 & 347.3 & 0.00 & 0.0764 & 0.1472 \\
Gamma & 345.6 & 347.5 & 1.20 & 0.1031 & 0.3061 \\
Weibull & 346.1 & 348.0 & 1.20 & 0.1059 & 0.3227 \\
Triangular & 349.4 & 351.3 & 6.80 & 0.2779 & 2.8428 \\
Kumaraswamy & 352.2 & 355.1 & 1.60 & 0.1251 & 0.4555 \\
\hline
\end{tabular}

Figure S7. Simulated Liquids Unloading Venting Duration (seconds), Automatic Plunger-Lift

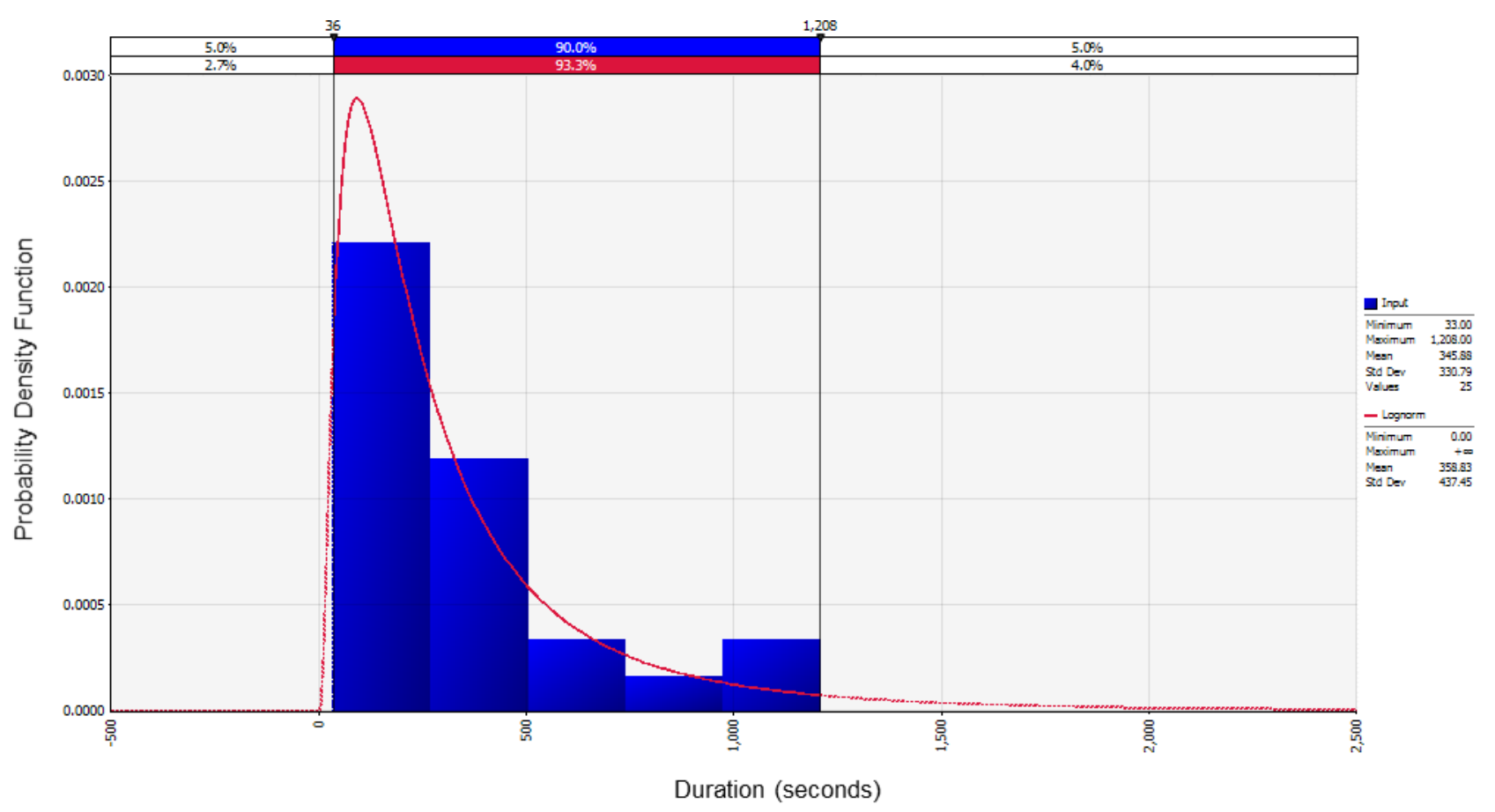

Table S14 compares multiple GOF indicators, obtained via fitting eight probability distributions against the venting frequency for automatic plunger-lift systems reported in Table S12. In this work, the venting frequency for automatic plunger-lift systems is characterized as a Weibull distribution with mean of $\sim 2450$ events/well-year and standard deviation of $\sim 1909$, see Figure S8. 
Table S14. Goodness of Fit Criteria, Automatic Plunger-Lifts, Venting Frequency

\begin{tabular}{llllll}
\hline Distribution & AIC & BIC & Chi-Squared & K-S & A-D \\
\hline Exponential & 442.2 & 443.3 & 4.80 & 0.1542 & 0.6354 \\
Lognormal & 443.4 & 445.3 & 3.20 & 0.1603 & 0.5471 \\
Invgauss & 444.3 & 446.2 & 8.00 & 0.2008 & 0.7356 \\
Loglogistic & 444.9 & 446.7 & 3.20 & 0.1423 & 0.5777 \\
Gamma & 442.1 & 444.0 & 2.80 & 0.1312 & 0.4256 \\
Weibull & 442.2 & 444.1 & 2.80 & 0.1342 & 0.4082 \\
Triangular & 442.0 & 443.9 & 8.00 & 0.2061 & 1.4515 \\
Kumaraswamy & 447.6 & 450.4 & 2.80 & 0.1334 & 0.3962 \\
\hline
\end{tabular}

Figure S8. Simulated Liquids Unloading Venting Frequency (vents/well-year), Automatic Plunger-Lift

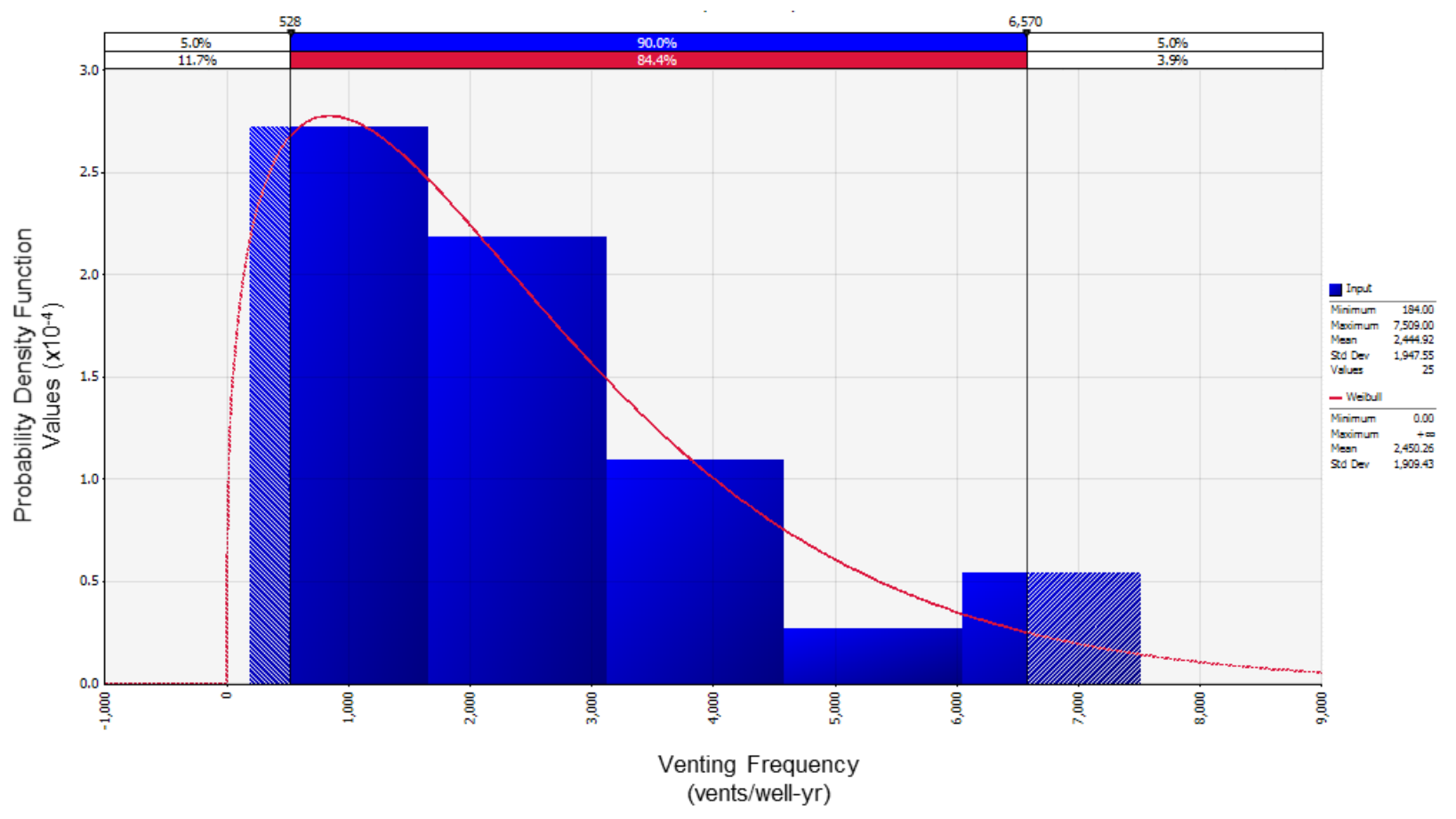




\section{S6. Venting Well Count}

It is important to note that not all non-plunger and plunger-lift equipped wells vent to the atmosphere, thus wells that vent represent a sub-set of total well count. The six liquids unloading scenarios evaluated in this work are highlighted in red, see Figure S9.

Figure S9. Wells disaggregated by well type, venting, and liquids unloading system.

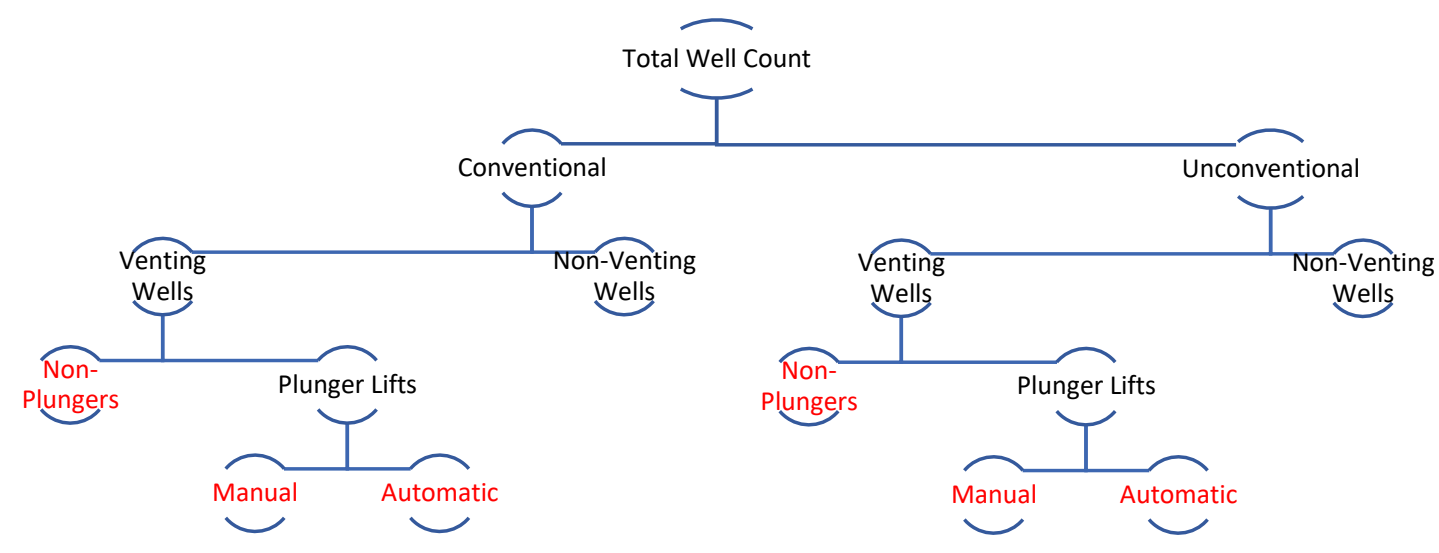

The venting well count $(\mathrm{Wi}, \mathrm{j})$ for the $i^{\text {th }}$ scenario and $j^{\text {th }}$ county is calculated as the product of the countylevel well count ( $\mathrm{W}_{\mathrm{DI}, \text { County }}$ ) obtained from DI desktop and fraction of non-plunger, manual plunger-lift, or automatic plunger-lift wells that vent for liquids unloading $\left(\mathrm{F}_{\mathrm{venting}}\right)$ derived from data reported to the 2016 GHGRP. It is important to note that $\left(F_{\text {venting }}\right)$ is calculated at the county-level $\left(F_{\text {venting,County }}\right)$, however in instances in which county-level data is unavailable or not reported, $F_{\text {vent }}$ is derived at the basin level ( $F_{\text {venting,Basin }}$,. The calculation of venting well count $(\mathrm{Wi}, \mathrm{j})$ is shown in $\operatorname{Eqn}(2)$.

$$
W_{i, j}=W_{D I, \text { County }} \times F_{\text {Venting }}
$$

County-level well count ( $\left.\mathrm{W}_{\mathrm{DI}, \text { County }}\right)$ is obtained from DI desktop, and stratified across conventional and unconventional well types. Data from the 2016 GHGRP is used to estimate the fraction of wells that vent for liquids unloading $\left(\mathrm{F}_{\text {venting }}\right)$ for non-plunger, manual plunger-lift, and automatic-plunger lifts. A detailed workflow for calculating the of wells that vent for liquids unloading at the county-level ( $\left.\mathrm{F}_{\text {venting,County }}\right)$ is provided in Figure S10. 
Figure S10. Detailed Workflow for calculating $\mathrm{F}_{\text {venting,County }}$

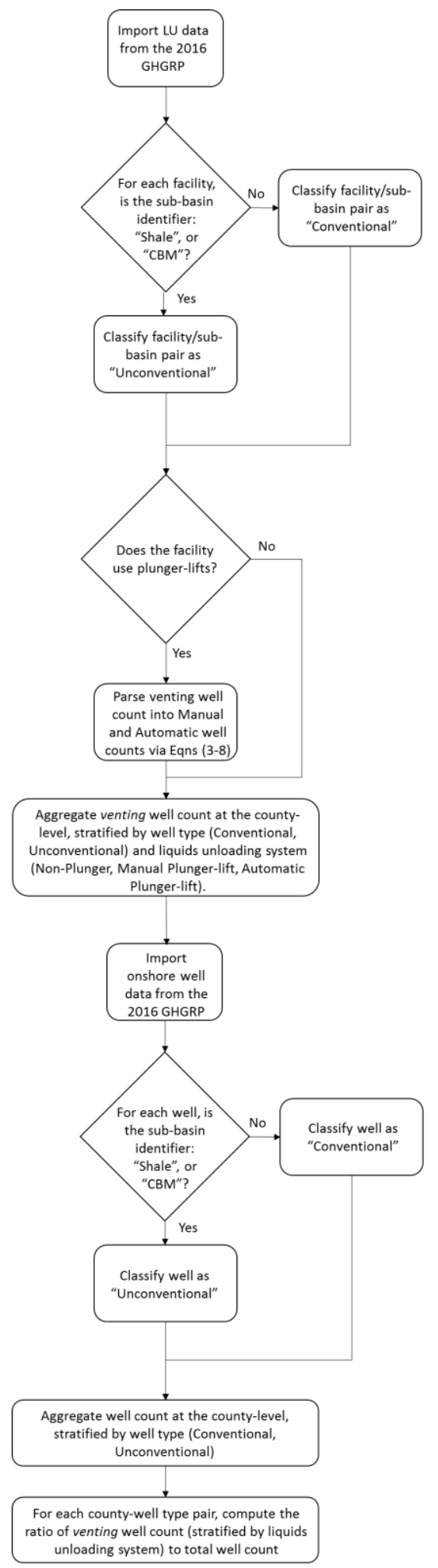


A summary of all onshore wells sampled in the 2016 GHGRP is publicly available via the data-set "EF_W_ONSHORE_WELLS". In this work, onshore natural gas wells reported by the GHGRP are matched to natural gas basins based on AAPG Geologic Provinces Codes. Wells with formation type "Coal seam" and "Shale gas" are considered unconventional, while wells with formation type "High permeability gas", "Oil", and "Other tight reservoir rock" are considered conventional. Basin-level well count $\left(\mathrm{W}_{\text {GHGRP,Basin }}\right)$ from the 2016 GHGRP, stratified by well type (conventional, unconventional) is provided in Table $\mathbf{S 1 5 .}$

Table S15. Basin-level well count ( $\left.\mathrm{W}_{\mathrm{GHGRP}, \mathrm{Basin}}\right)$ from the 2016 GHGRP

\begin{tabular}{llll}
\hline Basin & Conv. Wells & Unconv. Wells & Total \\
\hline Anadarko Basin & 38,145 & 2,031 & 40,176 \\
Appalachian Basin Eastern Overthrust & 40,451 & 18,664 & 59,115 \\
Appalachian Basin & 20,014 & 8,443 & 28,457 \\
Arkla Basin & 5,770 & 2,483 & 8,253 \\
Arkoma Basin & 6,207 & 3,068 & 9,275 \\
Chautauqua Platform & 94 & 291 & 385 \\
Denver Basin & 19,163 & 1,082 & 20,245 \\
East Texas Basin & 17,257 & 1,483 & 18,740 \\
Fort Worth Syncline & 2,029 & 6,644 & 8,673 \\
Green River Basin & 10,444 & 83 & 10,527 \\
Gulf Coast Basin & 23,128 & 5,868 & 28,996 \\
Permian Basin & 84,674 & 1,158 & 85,832 \\
Piceance Basin & 13,397 & 478 & 13,875 \\
San Juan Basin & 14,460 & 6,901 & 21,361 \\
South Oklahoma Folded Belt & 1,691 & 724 & 2,415 \\
Strawn Basin & 990 & 5,994 & 6,984 \\
Uinta Basin & 10,864 & 202 & 11,066 \\
Williston Basin & 16,762 & 6 & 16,768 \\
\hline Rocky Mountain & 85,090 & 8,752 & 93,842 \\
Gulf Coast & 46,155 & 9,834 & 55,989 \\
Mid-Continent & 133,830 & 19,910 & 153,740 \\
Northeast & 60,465 & 27,107 & 87,572 \\
\hline Aggregate & 325,540 & 65,603 & 391,143 \\
\hline
\end{tabular}

The GHGRP provides a detailed summary of wells that vent for liquids unloading throughout the 2016 reporting year, in the dataset "EF_W_LIQUIDS_UNLOAD_UNITS". In this work, facilities that vent for liquids unloading with formation type "Coal seam" and "Shale gas" are classified as unconventional, while facilities with formation type "High permeability gas", "Oil", and "Other tight reservoir rock" are considered conventional. Additionally, the 2016 GHGRP reports the number of non-plunger and plungerlifts wells that vent for liquids unloading, at the facility-level. However, the GHGRP does not distinguish between venting manual or automatic plunger-lift wells. This work estimates the number of automatic and manual plunger-lift systems for each facility-well type pair, using data from the GHGRP as model 
constraints. The augmented data structure of the GHGRP is provided in Figure S11, data from the GHGRP is mapped to county-level data obtained from DI desktop.

\section{Figure S11. Augmented Data Structure GHGRP}

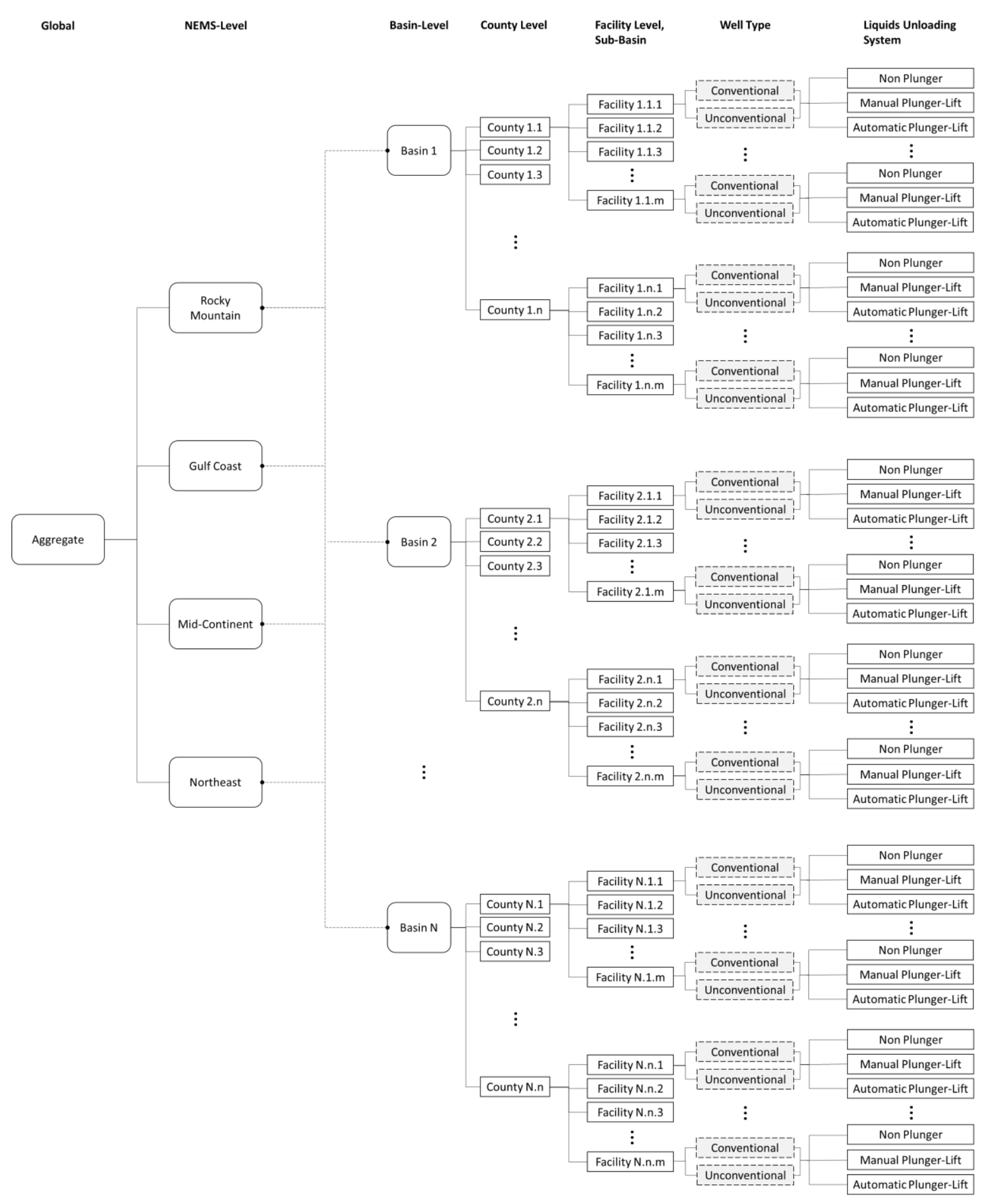


The following approach is used disaggregate facility-level venting plunger-lift well count, reported by the GHGRP, into venting automatic and manual plunger-lift well counts.

Let:

$\mathrm{X}=$ Venting automatic plunger-lift well count

$\mathrm{Y}=$ Venting manual plunger-lift well count

$\mathrm{Z}=$ Venting plunger-lift well count, as reported by GHGRP

$\mathrm{V}=$ Average liquids unloading event count, as reported by GHGRP

Vfauto $=$ Per well venting frequency for automatic plunger-lifts

Vfmanual $=$ Per well venting frequency for manual plunger-lifts

It is assumed that facility-level venting plunger-lift well count (Z), reported by the GHGRP, is composed of (X) venting automatic plunger lifts and (Y) manual plunger-lift wells, see Eqn (3).

$$
X+Y=Z
$$

The average liquid unloading event count (V), as reported by GHGRP, is thus a linear combination of per-well venting frequency $\left(\mathrm{Vf}_{\text {auto }}, \mathrm{Vf}_{\text {manual }}\right)$ and well count $(\mathrm{X}, \mathrm{Y})$ for automatic and manual plunger-lifts, respectively, see Eqn (4).

$$
V f_{\text {auto }}(X)+V f_{\text {manual }}(Y)=V
$$

Substitute for Y using Eqn (3), to yield Eqn (5)

$$
V f_{\text {auto }}(X)+V f_{\text {manual }}(Z-X)=V
$$

Rearranging, yields Eqn (6)

$$
X \times\left(V f_{\text {auto }}-V f_{\text {manual }}\right)=V-\left(V f_{\text {manual }} \times Z\right)
$$

Solving for X yields Eqn (7)

$$
X=\frac{V-\left(V f_{\text {manual }} \times Z\right)}{\left(V f_{\text {auto }}-V f_{\text {manual }}\right)}
$$

However, it is important to note that this approaches produces erroneous results in Eqn (7) for $X$ if $\left(V f_{\text {manual }} \times Z\right)>\mathrm{V}$ and in Eqn (3) for $\mathrm{Y}$ if $\mathrm{V}>\left(V f_{\text {auto }} \times Z\right)$. As such, the following conditions are applied to estimate the fraction of venting manual and automatic plunger-lifts wells: if $\left(V f_{\text {manual }} \times Z\right)>$ $\mathrm{V}$ then all wells are assumed to be manual plunger-lifts (i.e. $\mathrm{Y}=\mathrm{Z})$, if $\mathrm{V}>\left(V f_{\text {auto }} \times Z\right)$ then all wells are assumed to be automatic plunger-lifts (i.e. $\mathrm{X}=\mathrm{Z})$, if $\left(V f_{\text {auto }} \times Z\right)>\mathrm{V}>\left(V f_{\text {manual }} \times Z\right)$, then $\mathrm{X}$ is 
estimated using Eqn (7) and Y is estimated from Eqn (3). As well count is an integer value, X and Y are rounded to the nearest whole value, see Eqn (8). In this approach, variability in the venting frequency for manual and automatic plunger-lifts (see Figure S6 and Figure S8), is propagated into variability in venting automatic well count $(\mathrm{X})$ and venting manual well count $(\mathrm{Y})$.

$$
\text { If }\left\{\begin{array}{lll}
V f_{\text {manual }} \times Z>V, & X=0, & Y=Z \\
V f_{\text {auto }} \times Z>V>V f_{\text {manual }} \times Z, & X=\text { Round }\left(\frac{V-\left(V f_{\text {manual }} \times Z\right)}{\left(V f_{\text {auto }}-V f_{\text {manual }}\right)}\right), & Y=Z-X \\
V>V f_{\text {auto }} \times Z, & X=Z, & Y=0
\end{array}\right.
$$

Venting well count reported in the 2016 GHGRP, stratified by well type and liquids unloading system, is aggregated at both the county-level ( $\mathrm{W}_{\text {vent,GHGRP,County }}$ ) and basin-level ( $\mathrm{W}_{\text {vent,GHGRP,Basin }}$ ), and used to derived county-level $\left(\mathrm{F}_{\text {vent,County }}\right)$ and basin-level venting well fractions ( $\left.\mathrm{F}_{\text {vent,Basin }}\right)$, respectively. For brevity, only basin-level venting well counts are shown, and provided in Table S16. Uncertainty in the well count for manual and automatic plunger-lifts systems are reported as the $5^{\text {th }}$ and $95^{\text {th }}$ percentiles obtained via Monte-Carlo simulation.

Basin-level venting well fractions $\left(\mathrm{F}_{\mathrm{vent}, \mathrm{Basin}}\right)$ are provided in Table S17, and derived via the ratio of basinlevel venting well count $\left(\mathrm{W}_{\text {vent,GHGRP,Basin }}\right)$ reported in Table S16, to basin well count $\left(\mathrm{W}_{\mathrm{GHGRP}, \text { Basin }}\right)$

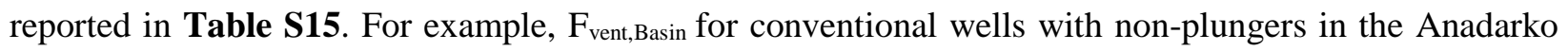
basin is the ratio of basin-level venting well count (259) to conventional well count $(38,145)$, thus $\mathrm{F}_{\text {vent,Basin }} \sim 0.68 \%$. 
Table S16. Basin-level venting well count ( $\mathrm{W}_{\text {vent,GHGRP,Basin }}$ ) inferred from the 2016 GHGRP

\begin{tabular}{|c|c|c|c|c|c|c|c|}
\hline Basin & $\begin{array}{l}\text { Conv. Non- } \\
\text { Plunger }\end{array}$ & $\begin{array}{l}\text { Conv. Manual } \\
\text { Plunger-Lift }\end{array}$ & $\begin{array}{l}\text { Conv. Automatic } \\
\text { Plunger-Lift }\end{array}$ & $\begin{array}{l}\text { Unconv. Non- } \\
\text { Plunger }\end{array}$ & $\begin{array}{l}\text { Unconv. Manual } \\
\text { Plunger-Lift }\end{array}$ & $\begin{array}{l}\text { Unconv. Automatic } \\
\text { Plunger-Lift }\end{array}$ & Total \\
\hline Anadarko Basin & $259(259,259)$ & $1177(1151,1188)$ & $16(5,42)$ & $98(98,98)$ & $101(101,101)$ & $0(0,0)$ & 1,651 \\
\hline Appalachian Basin $(160 \mathrm{~A})^{*}$ & $1548(1548,1548)$ & $3976(3901,4016)$ & $65(25,140)$ & $1554(1554,1554)$ & $1121(1096,1130)$ & $12(3,37)$ & 8,276 \\
\hline Appalachian Basin (160) & $313(313,313)$ & $1614(1601,1620)$ & $10(4,23)$ & $526(526,526)$ & $610(574,634)$ & $43(19,79)$ & 3,116 \\
\hline Arkla Basin & $1133(1133,1133)$ & $52(39,76)$ & $42(18,55)$ & $545(545,545)$ & $24(24,24)$ & $0(0,0)$ & 1,796 \\
\hline Arkoma Basin & $2069(2069,2069)$ & $419(323,517)$ & $290(192,386)$ & $385(385,385)$ & $337(302,361)$ & $51(27,86)$ & 3,551 \\
\hline Chautauqua Platform & $2(2,2)$ & $2(2,2)$ & $0(0,0)$ & $42(42,42)$ & $21(21,21)$ & $0(0,0)$ & 67 \\
\hline Denver Basin & $174(174,174)$ & $2351(2344,2354)$ & $3(0,10)$ & $4(4,4)$ & $0(0,0)$ & $0(0,0)$ & 2,532 \\
\hline East Texas Basin & $794(794,794)$ & $382(381,382)$ & $0(0,1)$ & $89(89,89)$ & $4(4,4)$ & $0(0,0)$ & 1,269 \\
\hline Fort Worth Syncline & $20(20,20)$ & $41(41,41)$ & $0(0,0)$ & $362(362,362)$ & $492(491,492)$ & $0(0,1)$ & 915 \\
\hline Green River Basin & $851(851,851)$ & $1867(1854,1874)$ & $8(1,21)$ & $3(3,3)$ & $0(0,0)$ & $0(0,0)$ & 2,729 \\
\hline Gulf Coast Basin & $1105(1105,1105)$ & $88(87,88)$ & $0(0,1)$ & $195(195,195)$ & $54(54,54)$ & $0(0,0)$ & 1,442 \\
\hline Permian Basin & $194(194,194)$ & $184(184,184)$ & $0(0,0)$ & $26(26,26)$ & $0(0,0)$ & $0(0,0)$ & 404 \\
\hline Piceance Basin & $343(343,343)$ & $3736(3721,3744)$ & $8(0,23)$ & $52(52,52)$ & $3(3,3)$ & $0(0,0)$ & 4,142 \\
\hline San Juan Basin & $728(728,728)$ & $3651(2239,4160)$ & $698(189,2109)$ & $161(161,161)$ & $190(175,201)$ & $16(5,31)$ & 5,444 \\
\hline South Oklahoma Folded Belt & $3(3,3)$ & $41(41,41)$ & $0(0,0)$ & $14(14,14)$ & $19(19,19)$ & $0(0,0)$ & 77 \\
\hline Strawn Basin & $13(13,13)$ & $9(9,9)$ & $0(0,0)$ & $356(356,356)$ & $865(864,866)$ & $1(0,2)$ & 1,244 \\
\hline Uinta Basin & $345(345,345)$ & $939(840,971)$ & $38(6,137)$ & $0(0,0)$ & $0(0,0)$ & $0(0,0)$ & 1,322 \\
\hline Williston Basin & $435(435,435)$ & $6(6,6)$ & $0(0,0)$ & $0(0,0)$ & $0(0,0)$ & $0(0,0)$ & 441 \\
\hline
\end{tabular}

*Appalachian Basin (160A): Appalachian Eastern Overthrust Basin

Venting well counts are reported as Mean (P5, P95) 
Table S17. Basin-level Venting Well Fractions ( $\left.F_{\text {vent,Basin }}\right)$

\begin{tabular}{|c|c|c|c|c|c|c|}
\hline Basin & Conv. Non-Plunger & $\begin{array}{l}\text { Conv. Manual Plunger- } \\
\text { Lift }\end{array}$ & $\begin{array}{l}\text { Conv. Automatic Plunger- } \\
\text { Lift }\end{array}$ & Unconv. Non-Plunger & $\begin{array}{l}\text { Unconv. Manual Plunger- } \\
\text { Lift }\end{array}$ & $\begin{array}{l}\text { Unconv. Automatic Plunger } \\
\text { Lift }\end{array}$ \\
\hline Anadarko Basin & $0.68 \%(0.68 \%, 0.68 \%)$ & $3.08 \%(3.02 \%, 3.11 \%)$ & $0.04 \%(0.01 \%, 0.11 \%)$ & $4.83 \%(4.83 \%, 4.83 \%)$ & $4.97 \%(4.97 \%, 4.97 \%)$ & $0 \%(0 \%, 0 \%)$ \\
\hline Appalachian Basin (160A) & $3.83 \%(3.83 \%, 3.83 \%)$ & $9.83 \%(9.64 \%, 9.93 \%)$ & $0.16 \%(0.06 \%, 0.35 \%)$ & $8.33 \%(8.33 \%, 8.33 \%)$ & $6.01 \%(5.87 \%, 6.05 \%)$ & $0.06 \%(0.02 \%, 0.2 \%)$ \\
\hline Appalachian Basin (160) & $1.56 \%(1.56 \%, 1.56 \%)$ & $8.06 \%(8 \%, 8.09 \%)$ & $0.05 \%(0.02 \%, 0.11 \%)$ & $6.23 \%(6.23 \%, 6.23 \%)$ & $7.23 \%(6.81 \%, 7.51 \%)$ & $0.5 \%(0.23 \%, 0.92 \%)$ \\
\hline Arkla Basin & $19.64 \%(19.64 \%, 19.64 \%)$ & $0.89 \%(0.68 \%, 1.33 \%)$ & $0.73 \%(0.29 \%, 0.95 \%)$ & $21.95 \%(21.95 \%, 21.95 \%)$ & $0.97 \%(0.97 \%, 0.97 \%)$ & $0 \%(0 \%, 0 \%)$ \\
\hline Arkoma Basin & $33.33 \%(33.33 \%, 33.33 \%)$ & $6.75 \%(5.2 \%, 8.38 \%)$ & $4.67 \%(3.04 \%, 6.22 \%)$ & $12.55 \%(12.55 \%, 12.55 \%)$ & $10.98 \%(9.88 \%, 11.77 \%)$ & $1.67 \%(0.88 \%, 2.77 \%)$ \\
\hline Chautauqua Platform & $2.13 \%(2.13 \%, 2.13 \%)$ & $2.13 \%(2.13 \%, 2.13 \%)$ & $0 \%(0 \%, 0 \%)$ & $14.43 \%(14.43 \%, 14.43 \%)$ & $7.22 \%(7.22 \%, 7.22 \%)$ & $0 \%(0 \%, 0 \%)$ \\
\hline Denver Basin & $0.91 \%(0.91 \%, 0.91 \%)$ & $12.27 \%(12.23 \%, 12.28 \%)$ & $0.02 \%(0 \%, 0.05 \%)$ & $0.37 \%(0.37 \%, 0.37 \%)$ & $0 \%(0 \%, 0 \%)$ & $0 \%(0 \%, 0 \%)$ \\
\hline East Texas Basin & $4.6 \%(4.6 \%, 4.6 \%)$ & $2.21 \%(2.21 \%, 2.21 \%)$ & $0 \%(0 \%, 0.01 \%)$ & $6 \%(6 \%, 6 \%)$ & $0.27 \%(0.27 \%, 0.27 \%)$ & $0 \%(0 \%, 0 \%)$ \\
\hline Fort Worth Syncline & $0.99 \%(0.99 \%, 0.99 \%)$ & $2.02 \%(2.02 \%, 2.02 \%)$ & $0 \%(0 \%, 0 \%)$ & $5.45 \%(5.45 \%, 5.45 \%)$ & $7.4 \%(7.39 \%, 7.41 \%)$ & $0 \%(0 \%, 0.02 \%)$ \\
\hline Green River Basin & $8.15 \%(8.15 \%, 8.15 \%)$ & $17.88 \%(17.74 \%, 17.94 \%)$ & $0.07 \%(0.01 \%, 0.21 \%)$ & $3.61 \%(3.61 \%, 3.61 \%)$ & $0 \%(0 \%, 0 \%)$ & $0 \%(0 \%, 0 \%)$ \\
\hline Gulf Coast Basin & $4.78 \%(4.78 \%, 4.78 \%)$ & $0.38 \%(0.38 \%, 0.38 \%)$ & $0 \%(0 \%, 0 \%)$ & $3.32 \%(3.32 \%, 3.32 \%)$ & $0.92 \%(0.92 \%, 0.92 \%)$ & $0 \%(0 \%, 0 \%)$ \\
\hline Permian Basin & $0.23 \%(0.23 \%, 0.23 \%)$ & $0.22 \%(0.22 \%, 0.22 \%)$ & $0 \%(0 \%, 0 \%)$ & $2.25 \%(2.25 \%, 2.25 \%)$ & $0 \%(0 \%, 0 \%)$ & $0 \%(0 \%, 0 \%)$ \\
\hline Piceance Basin & $2.56 \%(2.56 \%, 2.56 \%)$ & $27.89 \%(27.77 \%, 27.95 \%)$ & $0.06 \%(0 \%, 0.17 \%)$ & $10.88 \%(10.88 \%, 10.88 \%)$ & $0.63 \%(0.63 \%, 0.63 \%)$ & $0 \%(0 \%, 0 \%)$ \\
\hline San Juan Basin & $5.03 \%(5.03 \%, 5.03 \%)$ & $25.24 \%(15.43 \%, 28.79 \%)$ & $4.83 \%(1.28 \%, 14.64 \%)$ & $2.33 \%(2.33 \%, 2.33 \%)$ & $2.75 \%(2.54 \%, 2.91 \%)$ & $0.23 \%(0.07 \%, 0.45 \%)$ \\
\hline South Oklahoma Folded Belt & $0.18 \%(0.18 \%, 0.18 \%)$ & $2.42 \%(2.42 \%, 2.42 \%)$ & $0 \%(0 \%, 0 \%)$ & $1.93 \%(1.93 \%, 1.93 \%)$ & $2.62 \%(2.62 \%, 2.62 \%)$ & $0 \%(0 \%, 0 \%)$ \\
\hline Strawn Basin & $1.31 \%(1.31 \%, 1.31 \%)$ & $0.91 \%(0.91 \%, 0.91 \%)$ & $0 \%(0 \%, 0 \%)$ & $5.94 \%(5.94 \%, 5.94 \%)$ & $14.44 \%(14.41 \%, 14.45 \%)$ & $0.01 \%(0 \%, 0.03 \%)$ \\
\hline Uinta Basin & $3.18 \%(3.18 \%, 3.18 \%)$ & $8.64 \%(7.72 \%, 8.94 \%)$ & $0.35 \%(0.06 \%, 1.27 \%)$ & $0 \%(0 \%, 0 \%)$ & $0 \%(0 \%, 0 \%)$ & $0 \%(0 \%, 0 \%)$ \\
\hline Williston Basin & $2.6 \%(2.6 \%, 2.6 \%)$ & $0.04 \%(0.04 \%, 0.04 \%)$ & $0 \%(0 \%, 0 \%)$ & $0 \%(0 \%, 0 \%)$ & $0 \%(0 \%, 0 \%)$ & $0 \%(0 \%, 0 \%)$ \\
\hline
\end{tabular}

*Appalachian Basin (160A): Appalachian Eastern Overthrust Basin

reported

as

Mean

(P5,

P95) 
County-level venting well count $\left(\mathrm{W}_{\mathrm{i}, \mathrm{j}}\right)$ for non-plunger, manual plunger-lift, and automatic plunger-lift systems is constructed by applying venting well fractions $\left(F_{\text {vent }}\right)$ derived from the 2016 GHGRP, to county-level well count ( $\left.\mathrm{W}_{\mathrm{DI}, \mathrm{County}}\right)$ obtained from DI desktop. If there is a direct match in county-well type pairs between GHGRP and DI desktop, then county-level venting well fractions are applied $\left(\mathrm{F}_{\text {vent,County }}\right)$, otherwise basin-level venting well fractions $\left(\mathrm{F}_{\text {vent,Basin }}\right)$-see Table S17, are used. For brevity, only summary statistics (e.g. $5^{\text {th }}, 25^{\text {th }}, 50^{\text {th }}, 75^{\text {th }}, 95^{\text {th }}$ percentiles, and mean) for basin-level venting well count $\left(\mathrm{W}_{\text {vent,DI,Basin }}\right)$ for non-plunger, manual-plunger lift, and automatic plunger lift systems obtained via Monte-Carlo simulation are shown, and provided in Table S18, Table S19, and Table S20 respectively.

Table S18. Venting Well Count, Non-Plungers

\begin{tabular}{lllllll}
\hline Basins & P5 & P25 & P50 & Mean & P75 & P95 \\
\hline Anadarko Basin & 826 & 826 & 826 & 826 & 826 & 826 \\
Appalachian Basin (160A)* & 5,299 & 5,299 & 5,299 & 5,299 & 5,299 & 5,299 \\
Appalachian Basin (160) & 533 & 533 & 533 & 533 & 533 & 533 \\
Arkla Basin & 3,323 & 3,323 & 3,323 & 3,323 & 3,323 & 3,323 \\
Arkoma Basin & 2,900 & 2,900 & 2,900 & 2,900 & 2,900 & 2,900 \\
Chautauqua Platform & 344 & 344 & 344 & 344 & 344 & 344 \\
Denver Basin & 82 & 82 & 82 & 82 & 82 & 82 \\
East Texas Basin & 1,374 & 1,374 & 1,374 & 1,374 & 1,374 & 1,374 \\
Fort Worth Syncline & 317 & 317 & 317 & 317 & 317 & 317 \\
Green River Basin & 850 & 850 & 850 & 850 & 850 & 850 \\
Gulf Coast Basin & 1,542 & 1,542 & 1,542 & 1,542 & 1,542 & 1,542 \\
Permian Basin & 170 & 170 & 170 & 170 & 170 & 170 \\
Piceance Basin & 1,360 & 1,360 & 1,360 & 1,360 & 1,360 & 1,360 \\
San Juan Basin & 1,216 & 1,216 & 1,216 & 1,216 & 1,216 & 1,216 \\
South Oklahoma Folded Belt & 20 & 20 & 20 & 20 & 20 & 20 \\
Strawn Basin & 454 & 454 & 454 & 454 & 454 & 454 \\
Uinta Basin & 171 & 171 & 171 & 171 & 171 & 171 \\
Williston Basin & 477 & 477 & 477 & 477 & 477 & 477 \\
\hline Rocky Mountain & 4,156 & 4,156 & 4,156 & 4,156 & 4,156 & 4,156 \\
Gulf Coast & 6,238 & 6,238 & 6,238 & 6,238 & 6,238 & 6,238 \\
Mid-Continent & 5,030 & 5,030 & 5,030 & 5,030 & 5,030 & 5,030 \\
Northeast & 5,832 & 5,832 & 5,832 & 5,832 & 5,832 & 5,832 \\
\hline Aggregate & 21,257 & 21,257 & 21,257 & 21,257 & 21,257 & 21,257 \\
\hline Aan Basin (160A): Appalachian Eastern Overthrust Basin & & & & &
\end{tabular}


Table S19. Venting Well Count, Manual Plunger-Lifts

\begin{tabular}{lllllll}
\hline Basins & P5 & P25 & P50 & Mean & P75 & P95 \\
\hline Anadarko Basin & 2,069 & 2,111 & 2,122 & 2,115 & 2,128 & 2,133 \\
Appalachian Basin (160A)* & 8,497 & 8,653 & 8,698 & 8,670 & 8,727 & 8,753 \\
Appalachian Basin (160) & 3,171 & 3,187 & 3,195 & 3,193 & 3,201 & 3,207 \\
Arkla Basin & 114 & 115 & 128 & 148 & 182 & 215 \\
Arkoma Basin & 2,398 & 2,488 & 2,565 & 2,561 & 2,637 & 2,713 \\
Chautauqua Platform & 206 & 206 & 206 & 206 & 206 & 206 \\
Denver Basin & 635 & 637 & 637 & 637 & 638 & 638 \\
East Texas Basin & 427 & 428 & 428 & 428 & 428 & 428 \\
Fort Worth Syncline & 497 & 497 & 497 & 497 & 497 & 497 \\
Green River Basin & 648 & 655 & 656 & 655 & 657 & 658 \\
Gulf Coast Basin & 230 & 231 & 231 & 231 & 231 & 231 \\
Permian Basin & 177 & 177 & 177 & 177 & 177 & 177 \\
Piceance Basin & 826 & 828 & 829 & 828 & 829 & 829 \\
San Juan Basin & 3,343 & 5,138 & 5,623 & 5,307 & 5,853 & 6,009 \\
South Oklahoma Folded Belt & 70 & 70 & 70 & 70 & 70 & 70 \\
Strawn Basin & 1,032 & 1,034 & 1,034 & 1,034 & 1,034 & 1,034 \\
Uinta Basin & 579 & 616 & 623 & 616 & 626 & 628 \\
Williston Basin & 0 & 0 & 0 & 0 & 0 & 0 \\
\hline Rocky Mountain & 6,071 & 7,869 & 8,358 & 8,043 & 8,588 & 8,748 \\
Gulf Coast & 772 & 773 & 786 & 807 & 841 & 873 \\
Mid-Continent & 6,489 & 6,586 & 6,665 & 6,660 & 6,738 & 6,817 \\
Northeast & 11,689 & 11,845 & 11,890 & 11,863 & 11,920 & 11,950 \\
\hline Aggregate & 25,358 & 27,177 & 27,675 & 27,373 & 27,925 & 28,151 \\
\hline Appalachian Basin (160A): Appalachian Eastern Overthrust Basin & & & & &
\end{tabular}


Table S20. Venting Well Count, Automatic Plunger-Lifts

\begin{tabular}{lllllll}
\hline Basins & P5 & P25 & P50 & Mean & P75 & P95 \\
\hline Anadarko Basin & 8 & 13 & 19 & 26 & 29 & 72 \\
Appalachian Basin (160A) & 60 & 86 & 115 & 143 & 160 & 316 \\
Appalachian Basin (160) & 10 & 16 & 22 & 24 & 30 & 46 \\
Arkla Basin & 49 & 81 & 136 & 115 & 149 & 149 \\
Arkoma Basin & 298 & 375 & 446 & 450 & 523 & 614 \\
Chautauqua Platform & 0 & 0 & 0 & 0 & 0 & 0 \\
Denver Basin & 0 & 0 & 0 & 1 & 1 & 3 \\
East Texas Basin & 0 & 0 & 0 & 0 & 0 & 1 \\
Fort Worth Syncline & 0 & 0 & 0 & 0 & 0 & 1 \\
Green River Basin & 1 & 1 & 2 & 3 & 3 & 10 \\
Gulf Coast Basin & 0 & 0 & 0 & 0 & 0 & 0 \\
Permian Basin & 0 & 0 & 0 & 0 & 0 & 0 \\
Piceance Basin & 0 & 0 & 0 & 1 & 1 & 3 \\
San Juan Basin & 283 & 439 & 668 & 984 & 1,153 & 2,948 \\
South Oklahoma Folded Belt & 0 & 0 & 0 & 0 & 0 & 0 \\
Strawn Basin & 0 & 0 & 0 & 1 & 0 & 2 \\
Uinta Basin & 2 & 4 & 7 & 14 & 14 & 52 \\
Williston Basin & 0 & 0 & 0 & 0 & 0 & 0 \\
\hline Rocky Mountain & 299 & 459 & 689 & 1,004 & 1,177 & 2,969 \\
Gulf Coast & 49 & 81 & 136 & 115 & 149 & 150 \\
Mid-Continent & 320 & 399 & 472 & 477 & 551 & 647 \\
Northeast & 80 & 110 & 140 & 167 & 185 & 340 \\
\hline Aggregate & 985 & 1,211 & 1,461 & 1,763 & 1,958 & 3,767 \\
\hline
\end{tabular}

*Appalachian Basin (160A): Appalachian Eastern Overthrust Basin

\section{S7. Standard Flow Rate (SFR)}

The per-well standard flow rate (SFR) of natural gas is estimated by converting annual 2016 county-level natural gas production ( $\mathrm{scf}$ year-1) obtained via DI desktop, to hourly production ( $\mathrm{scf} \mathrm{hr}^{-1}$ ) and dividing by county-level well count. It is important to note that there are 8,760 hours in a typical calendar year (365 days), however 2016 was a leap-year (366 days) and thus a conversion factor of 8,784 hrs/year is used in the analysis. SFR is disaggregated by conventional and unconventional well types and shown in Eqn (9).

$$
S F R=\left\{\begin{aligned}
\text { Conventional } & =\frac{\text { Conventional NG Production }\left(\frac{\text { scf }}{\text { year }}\right)}{\text { Conventional Well Count }(\# \text { wells })} \times\left(\frac{\text { year }}{8784 \text { hrs }}\right) \\
\text { Unconventional } & =\frac{\text { Unconventional NG Production }\left(\frac{\text { scf }}{\text { year }}\right)}{\text { Unonventional Well Count }(\# \text { wells })} \times\left(\frac{\text { year }}{8784 \text { hrs }}\right)
\end{aligned}\right.
$$




\section{S8. Volumetric Fraction of Methane in Natural Gas ( $\left.\mathrm{V}_{\mathrm{Frac}, \mathrm{CH} 4}\right)$}

Triangular distributions are used to parameterize the volumetric fraction of methane in natural gas $\left(\mathrm{V}_{\mathrm{Fraa}, \mathrm{CH} 4}\right)$, based on data reported in Allen et al. 2015 and provided in Table S6, Table S9, Table S12. Expected values for $\mathrm{V}_{\text {Frac,CH4 }}$ are calculated as the average over each NEMS region (e.g. Northeast, Gulf Coast, Mid-Continent, and Rocky Mountain).

Table S21. Volumetric Fraction of Methane in Natural Gas ( $\left.\mathrm{V}_{\mathrm{Fra}, \mathrm{CH} 4}\right)$ by NEMS Region

\begin{tabular}{llll}
\hline NEMS Region & \multicolumn{3}{c}{ Volumetric Fraction of Methane in Natural Gas (\%) } \\
\hline Distribution Type: & \multicolumn{3}{c}{ Triangular Distribution } \\
& Min & Max & Expected \\
Rocky Mountain & $75.70 \%$ & $91.50 \%$ & $83.26 \%$ \\
Gulf Coast & $80.70 \%$ & $96.10 \%$ & $92.17 \%$ \\
Mid-Continent & $72.80 \%$ & $97.90 \%$ & $87.38 \%$ \\
Northeast & $95.70 \%$ & $97.30 \%$ & $96.22 \%$ \\
\hline
\end{tabular}

\section{S9. Greenhouse Gas Reporting Program (GHGRP)}

The 2016 GHGRP provides average values for well parameters (CD, TD, WD, and SP) reported at the facility-level. However, data provided by the GHGRP is largely limited to well casing diameter and tubing diameter, with sparse to no entries for well depth or shut-in pressure. In this work, facility-level data for CD and TD reported in the GHGRP, are mapped to county-level data obtained from DI desktop. Triangular distributions for well casing diameter and well tubing diameter are constructed at the countylevel, by considering the minimum, maximum and expected value (venting well weighted average) across all facilities that lie within said county. In cases where there is no direct mapping between facility level data reported in the GHGRP and county-level data obtained from DI desktop, basin-wide minimum, maximum and expected values are used the parameterize the triangular distribution(s). Additionally, Range Resources Appalachia LLC, in Venango PA, report a tubing diameter of 150 inches, orders of magnitude higher than commonly reported values in GHGRP. It is assumed that this data point is erroneous and thus was removed from the dataset.

\section{S10. API/ANGA Survey}

In this work, data from the API \& ANGA 2012 industrial survey is used to fill data gaps that may arise in the 2016 GHGRP dataset. API/ANGA reports data for well casing diameter, tubing diameter, well depth, and shut in pressure, across five NEMS regions (Northeast, Gulf Coast, Mid-Continent, Southwest, and Rocky Mountain), two well types (conventional and unconventional), and two broad liquids unloading technologies (non-plunger, plunger-lift systems). Triangular distributions are used to characterize well casing diameter, tubing diameter, well depth, and shut-in pressure, based on min, max, and expected values (venting well weighted average). A detailed example is provided below for clarity, sample data for liquids unloading for conventional gas wells without plunger lifts obtained directly from the API/ANGA survey is provided in Table $\mathbf{S 2 2}$. 
Table S22. Well parameters for conventional gas wells without plunger lifts

\begin{tabular}{llllllll}
\hline Well Parameter & \multicolumn{2}{l}{ Northeast } & Gulf Coast & Mid-Continent & Southwest \\
\hline Venting Gas Wells & 190 & 916 & 12 & 6 & 1 & 38 & 220 \\
Average Casing Diameter, (in) & 5 & 4.5 & 5.5 & 3.65 & 4.83 & 4 & 5.5 \\
Average well depth, (ft) & 3,375 & 3,448 & 10,000 & 19,334 & 7,033 & 4,269 & 8,000 \\
Average surface pressure, (psig) & 85 & 50 & 65.2 & 224 & 25.5 & 60.8 & 100 \\
\hline
\end{tabular}

Based on Table C-1 on pg. 41 in ref ${ }^{2}$

Using data from Table S22, the minimum and maximum value for the casing diameter (in) for conventional wells without plunger-lifts in the Northeast is 4.5 inches and 5 inches, respectively. The expected value for the casing diameter for conventional gas wells without plunger lifts in the Northeast is calculated as the average casing diameter weighted by the venting well count, and is provided in Eqn (10).

$$
C D=5 \times \frac{190}{(190+916)}+4.5 \times \frac{916}{(190+916)}=\sim 4.59
$$

For regions with null data (e.g. Table S22 does not report well parameters for NEMS region: Rocky Mountain), aggregate values are used. Aggregate values are constructed based on the minimum, maximum, and expected values (venting well-weighted average) across all NEMS regions. For example, the minimum and maximum value for the casing diameter (in) for conventional wells without plungerlifts, across all NEMS regions, is 3.65 inches and 5.5 inches, respectively. The expected value for the casing diameter for conventional gas wells without plunger lifts across all NEMS regions is calculated as the average casing diameter weighted by the venting well count, and is provided in Eqn (11).

$$
C D=5 \times \frac{190}{1383}+4.5 \times \frac{916}{1383}+5.5 \times \frac{12}{1383}+3.65 \times \frac{6}{1383}+4.83 \times \frac{1}{1383}+4 \times \frac{38}{1383}+5.5 \times \frac{220}{1383}=\sim 4.72
$$

A similar approach is used when estimating other well parameters (e.g. Tubing Diameter, Well Depth, and Shut-In Pressure).

\section{S10.1 Casing Diameter (CD)}

Table S23. Casing diameter, in units of inches, for non-plunger systems ${ }^{2}$

\begin{tabular}{lllllll}
\hline \multicolumn{1}{c}{ NEMS Region } & \multicolumn{3}{c}{ Conventional Wells } & \multicolumn{3}{c}{ Unconventional Wells } \\
\hline Distribution Type: & \multicolumn{3}{c}{ Triangular Distribution } & \multicolumn{3}{c}{ Triangular Distribution } \\
& Min & Max & Expected & Min & Max & Expected \\
Northeast & 4.5 & 5.0 & 4.59 & 4.5 & 4.5 & 4.500 \\
Gulf Coast & 3.65 & 5.5 & 4.88 & 4.5 & 10.75 & 5.616 \\
Mid-Continent & 4.0 & 4.83 & 4.02 & 4.92 & 5.5 & 5.374 \\
Southwest & 5.5 & 5.5 & 5.50 & 5.0 & 9.625 & 9.462 \\
Rocky Mountain & - & - & - & 2.375 & 4.7 & 4.078 \\
Aggregate & 3.65 & 5.50 & 4.719 & 2.375 & 10.75 & 5.734 \\
\hline
\end{tabular}




\section{S10.2 Tubing Diameter (TD)}

Table S24. Tubing diameter, in units of inches, for plunger-lift systems ${ }^{2}$

\begin{tabular}{lllllll}
\hline \multicolumn{1}{c}{ NEMS Region } & \multicolumn{3}{c}{ Conventional Wells } & \multicolumn{3}{c}{ Unconventional Wells } \\
\hline Distribution Type: & \multicolumn{3}{c}{ Triangular Distribution } & \multicolumn{3}{c}{ Triangular Distribution } \\
& Min & Max & Expected & Min & Max & Expected \\
Northeast & 2.0 & 2.375 & 2.29 & 2.375 & 2.375 & 2.38 \\
Gulf Coast & - & - & - & 2.375 & 2.375 & 2.38 \\
Mid-Continent & 1.995 & 2.375 & 2.02 & 2.375 & 4.11 & 3.19 \\
Southwest & - & - & - & 1.995 & 2.375 & 2.29 \\
Rocky Mountain & - & - & - & 1.92 & 2.375 & 2.30 \\
Aggregate & 1.995 & 2.375 & 2.14 & 1.92 & 4.11 & 2.38 \\
\hline
\end{tabular}

S10.3 Well Depth (WD)

Table S25. Well depth, in units of feet, for non-plunger systems ${ }^{2}$

\begin{tabular}{lllllll}
\hline \multicolumn{1}{c}{ NEMS Region } & \multicolumn{3}{c}{ Conventional Wells } & \multicolumn{3}{c}{ Unconventional Wells } \\
\hline Distribution Type: & \multicolumn{3}{c}{ Triangular Distribution } & \multicolumn{3}{c}{ Triangular Distribution } \\
& Min & Max & Expected & Min & Max & Expected \\
Northeast & 3,375 & 3,448 & 3,435 & 4,845 & 4,845 & 4,845 \\
Gulf Coast & 10,000 & 19,334 & 13,111 & 6,000 & 16,000 & 9,934 \\
Mid-Continent & 4,269 & 7,033 & 4,340 & 3,911 & 11,000 & 7,836 \\
Southwest & 8,000 & 8,000 & 8,000 & 8,000 & 15,000 & 8,786 \\
Rocky Mountain & - & - & - & 10,844 & 11,597 & 11,105 \\
Aggregate & 3,375 & 19,334 & 4,313 & 3,911 & 16,000 & 8,005 \\
\hline
\end{tabular}

Table S26. Well depth, in units of feet, for plunger-lift systems ${ }^{2}$

\begin{tabular}{lllllll}
\hline \multicolumn{1}{c}{ NEMS Region } & \multicolumn{3}{c}{ Conventional Wells } & \multicolumn{3}{c}{ Unconventional Wells } \\
\hline Distribution Type: & \multicolumn{3}{c}{ Triangular Distribution } & \multicolumn{3}{c}{ Triangular Distribution } \\
& Min & Max & Expected & Min & Max & Expected \\
Northeast & 3,375 & 3,448 & 3,431 & 2,500 & 7,000 & 4,290 \\
Gulf Coast & - & - & - & 8,500 & 16,000 & 11,098 \\
Mid-Continent & 4,269 & 9,500 & 4,598 & 3,911 & 10,293 & 6,638 \\
Southwest & - & - & - & 6,800 & 8,725 & 7,244 \\
Rocky Mountain & - & - & - & 7,400 & 11,164 & 8,968 \\
Aggregate & 3,375 & 9,500 & 4,077 & 2,500 & 16,000 & 7,913 \\
\hline
\end{tabular}




\section{S10.4 Shut-in Pressure (SP)}

Table S27. Shut-in pressure (psia), in units of psi, for non-plunger systems ${ }^{2}$

\begin{tabular}{lllllll}
\hline NEMS Region & \multicolumn{3}{c}{ Conventional Wells } & \multicolumn{3}{c}{ Unconventional Wells } \\
\hline Distribution Type: & \multicolumn{3}{c}{ Triangular Distribution } & \multicolumn{3}{c}{ Triangular Distribution } \\
& Min & Max & Expected & Min & Max & Expected \\
Northeast & 64.7 & 99.7 & 70.7 & 136.3 & 136.3 & 136.3 \\
Gulf Coast & 79.9 & 238.7 & 132.8 & 29.7 & 554.7 & 176.1 \\
Mid-Continent & 40.2 & 75.5 & 74.6 & 94.7 & 214.7 & 148.1 \\
Southwest & 114.7 & 114.7 & 114.7 & 64.7 & 530.7 & 514.9 \\
Rocky Mountain & - & - & - & 212.7 & 490.7 & 262.5 \\
Aggregate & 40.2 & 238.7 & 78.6 & 29.7 & 554.7 & 218.8 \\
\hline
\end{tabular}

The API \& ANGA survey report shut in pressure (psig) in units of psi. Shut-in pressure is converted from psig to psia, via adding atmospheric pressure (14.7 psi).

Table S28. Shut-in pressure (psia), in units of psi, for plunger-lift systems ${ }^{2}$

\begin{tabular}{lllllll}
\hline \multicolumn{1}{c}{ NEMS Region } & \multicolumn{3}{c}{ Conventional Wells } & \multicolumn{3}{c}{ Unconventional Wells } \\
\hline Distribution Type: & \multicolumn{2}{c}{ Triangular Distribution } & \multicolumn{2}{c}{ Triangular Distribution } \\
& Min & Max & Expected & Min & Max & Expected \\
Northeast & 64.7 & 99.7 & 72.8 & 136.3 & 214.7 & 155.8 \\
Gulf Coast & - & - & - & 29.7 & 554.7 & 90.4 \\
Mid-Continent & 40.2 & 514.7 & 100.0 & 89.39 & 113.45 & 102.2 \\
Southwest & - & - & - & 124.7 & 530.7 & 218.4 \\
Rocky Mountain & - & - & - & 164.7 & 304.7 & 207.1 \\
Aggregate & 40.2 & 514.7 & 87.9 & 29.7 & 554.7 & 185.1
\end{tabular}

The API \& ANGA survey report shut in pressure (psig) in units of psi. Shut-in pressure is converted from psig to psia, via adding atmospheric pressure (14.7 psi). 


\section{S11. Throughput Normalized Methane Emissions (TNME)}

Simulated throughput normalized methane emissions (TNME) from natural gas liquids unloading is provided in

Table S29, and compared against a composite TNME $_{\text {GHGRP }}$ inferred from the 2016 GHGRP.

Table S29. TNME from Natural Gas Liquids Unloading, and comparison with a composite TNME $_{\text {GHGRP }}$ inferred from the 2016 GHGRP

\begin{tabular}{lllllll|l}
\hline Basins & P5 & P25 & P50 & Mean & P75 & P95 & *TNMEGHGRP \\
\hline Anadarko Basin & $0.019 \%$ & $0.026 \%$ & $0.032 \%$ & $0.038 \%$ & $0.042 \%$ & $0.072 \%$ & $0.0060 \%$ \\
Appalachian Eastern Overthrust & $0.018 \%$ & $0.027 \%$ & $0.038 \%$ & $0.059 \%$ & $0.059 \%$ & $0.14 \%$ & $0.018 \%$ \\
Appalachian Basin & $0.042 \%$ & $0.055 \%$ & $0.068 \%$ & $0.077 \%$ & $0.087 \%$ & $0.14 \%$ & $0.012 \%$ \\
Arkla Basin & $0.060 \%$ & $0.10 \%$ & $0.14 \%$ & $0.19 \%$ & $0.22 \%$ & $0.46 \%$ & $0.015 \%$ \\
Arkoma Basin & $0.13 \%$ & $0.17 \%$ & $0.23 \%$ & $0.26 \%$ & $0.30 \%$ & $0.51 \%$ & $0.074 \%$ \\
Chautauqua Platform & $0.0057 \%$ & $0.011 \%$ & $0.018 \%$ & $0.028 \%$ & $0.031 \%$ & $0.078 \%$ & $0.0060 \%$ \\
Denver Basin & $0.042 \%$ & $0.063 \%$ & $0.089 \%$ & $0.12 \%$ & $0.13 \%$ & $0.28 \%$ & $0.032 \%$ \\
East Texas Basin & $0.043 \%$ & $0.068 \%$ & $0.10 \%$ & $0.13 \%$ & $0.15 \%$ & $0.33 \%$ & $0.018 \%$ \\
Fort Worth Syncline & $0.0061 \%$ & $0.012 \%$ & $0.021 \%$ & $0.036 \%$ & $0.038 \%$ & $0.11 \%$ & $0.0040 \%$ \\
Green River Basin & $0.0095 \%$ & $0.018 \%$ & $0.028 \%$ & $0.044 \%$ & $0.048 \%$ & $0.12 \%$ & $0.017 \%$ \\
Gulf Coast Basin & $0.015 \%$ & $0.021 \%$ & $0.029 \%$ & $0.039 \%$ & $0.042 \%$ & $0.091 \%$ & $0.0025 \%$ \\
Permian Basin & $0.0036 \%$ & $0.0054 \%$ & $0.0074 \%$ & $0.0093 \%$ & $0.011 \%$ & $0.021 \%$ & $0.00064 \%$ \\
Piceance Basin & $0.0085 \%$ & $0.021 \%$ & $0.047 \%$ & $0.15 \%$ & $0.13 \%$ & $0.54 \%$ & $0.0093 \%$ \\
San Juan Basin & $0.067 \%$ & $0.13 \%$ & $0.22 \%$ & $0.38 \%$ & $0.42 \%$ & $1.20 \%$ & $0.087 \%$ \\
South Oklahoma Folded Belt & $0.0024 \%$ & $0.0044 \%$ & $0.0070 \%$ & $0.013 \%$ & $0.012 \%$ & $0.036 \%$ & $0.0010 \%$ \\
Strawn Basin & $0.0058 \%$ & $0.0128 \%$ & $0.024 \%$ & $0.049 \%$ & $0.048 \%$ & $0.15 \%$ & $0.0077 \%$ \\
Uinta Basin & $0.0084 \%$ & $0.0193 \%$ & $0.035 \%$ & $0.061 \%$ & $0.066 \%$ & $0.19 \%$ & $0.051 \%$ \\
Williston Basin & $0.0021 \%$ & $0.0053 \%$ & $0.012 \%$ & $0.036 \%$ & $0.029 \%$ & $0.13 \%$ & $0.015 \%$ \\
\hline Rocky Mountain & $0.044 \%$ & $0.072 \%$ & $0.11 \%$ & $0.16 \%$ & $0.18 \%$ & $0.42 \%$ & $0.035 \%$ \\
Gulf Coast & $0.049 \%$ & $0.068 \%$ & $0.088 \%$ & $0.11 \%$ & $0.12 \%$ & $0.21 \%$ & $0.012 \%$ \\
Mid-Continent & $0.051 \%$ & $0.066 \%$ & $0.080 \%$ & $0.091 \%$ & $0.10 \%$ & $0.16 \%$ & $0.021 \%$ \\
Northeast & $0.020 \%$ & $0.028 \%$ & $0.039 \%$ & $0.060 \%$ & $0.059 \%$ & $0.14 \%$ & $0.017 \%$ \\
\hline Aggregate & $0.053 \%$ & $0.066 \%$ & $0.080 \%$ & $0.092 \%$ & $0.10 \%$ & $0.16 \%$ & $0.020 \%$ \\
\hline TNmE & 0.030 & & \\
\hline
\end{tabular}

*TNME and TNME $\mathrm{GHGRP}_{\text {are calculated based allocated methane emissions }}$

$\mathrm{TNME}_{\mathrm{GHGRP}}$ is derived from several data-elements reported to the GHGRP including (1) facility-level natural gas production, (2) salable oil production, and (3) methane emissions from liquids unloading. Facilities that report to the GHGRP are mapped to natural gas basins and NEMS regions, and assigned a corresponding volumetric concentration of methane in produced natural gas $\left(\mathrm{V}_{\mathrm{Frac}, \mathrm{CH} 4}\right)$, see Table $\mathbf{S 2 1}$. For calculation of $\mathrm{TNME}_{\mathrm{GHGRP}}$, allocation factors are constructed at the facility-level, and used to apportion methane emissions from natural gas liquids unloading across the methane component of produced natural gas, natural gas liquids and condensates $\left(\mathrm{C}_{2+}\right)$, and crude oil. Augmented facility-level

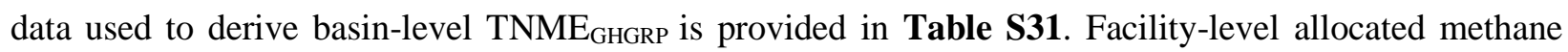
emissions from liquids unloading, as well as methane component of produced natural gas, is aggregated to the basin-level and used to derive basin-level TNME $E_{\text {GHGRP, }}$ see Table S30. The parameters in these tables are in terms of metric tonnes (MT), thousand barrels (Mbbl), and giga-cubic feet (GCF). 
Table S30. Augmented basin-level data used to derive TNME $_{\text {GHGRP }}$

\begin{tabular}{|c|c|c|c|c|c|c|}
\hline Basins & $\begin{array}{l}\text { Salable Oil } \\
\text { (Mbbl) }\end{array}$ & $\begin{array}{l}\text { Natural Gas } \\
\text { Produced (GCF) }\end{array}$ & $\begin{array}{l}\text { Methane Component of } \\
\text { Produced Natural Gas (MT } \\
\text { CH4) }\end{array}$ & $\begin{array}{l}\text { Methane Emissions } \\
\text { unallocated, (MT } \\
\text { CH4) }\end{array}$ & $\begin{array}{l}\text { Methane Emissions } \\
\text { (allocated), (MT } \\
\text { CH4) }\end{array}$ & $\begin{array}{l}\text { TNME }_{\text {GHGRP }} \\
(\%)\end{array}$ \\
\hline Anadarko Basin & 63,847 & 1,316 & $23,000,486$ & 2,048 & 1,378 & $0.0060 \%$ \\
\hline Appalachian Eastern Overthrust & 16,730 & 6,497 & $127,256,507$ & 23,907 & 22,468 & $0.018 \%$ \\
\hline Appalachian Basin & 1,930 & 237 & $4,650,906$ & 616 & 573 & $0.012 \%$ \\
\hline Arkla Basin & 2,863 & 1,307 & $23,809,969$ & 4,147 & 3,577 & $0.015 \%$ \\
\hline Arkoma Basin & 60 & 1,271 & $22,218,955$ & 19,510 & 16,432 & $0.074 \%$ \\
\hline Chautauqua Platform & 680 & 21 & 368,584 & 150 & 117 & $0.0060 \%$ \\
\hline Denver Basin & 85,202 & 581 & $9,850,291$ & 1,320 & 594 & $0.032 \%$ \\
\hline East Texas Basin & 2,227 & 1,578 & $28,741,927$ & 5,911 & 5,090 & $0.018 \%$ \\
\hline Fort Worth Syncline & 1,996 & 446 & $7,800,717$ & 372 & 309 & $0.0040 \%$ \\
\hline Green River Basin & 30,880 & 893 & $15,140,595$ & 3,262 & 2,534 & $0.017 \%$ \\
\hline Gulf Coast Basin & 429,033 & 1,565 & $28,509,398$ & 1,785 & 706 & $0.0025 \%$ \\
\hline Permian Basin & 210,129 & 804 & $14,044,187$ & 211 & 90 & $0.00064 \%$ \\
\hline Piceance Basin & 1,743 & 899 & $15,256,019$ & 1,765 & 1,423 & $0.0093 \%$ \\
\hline San Juan Basin & 5,125 & 987 & $16,739,950$ & 18,169 & 14,642 & $0.087 \%$ \\
\hline South Oklahoma Folded Belt & 7,253 & 456 & $7,973,809$ & 108 & 82 & $0.0010 \%$ \\
\hline Strawn Basin & 34 & 927 & $16,197,202$ & 1,473 & 1,241 & $0.0077 \%$ \\
\hline Uinta Basin & 809,654 & 282 & $4,774,911$ & 3,973 & 2,443 & $0.051 \%$ \\
\hline Williston Basin & 35,211 & 79 & $1,334,963$ & 328 & 204 & $0.015 \%$ \\
\hline Rocky Mountain & 967,814 & 3,720 & $63,096,729$ & 28,816 & 21,840 & $0.035 \%$ \\
\hline Gulf Coast & 434,123 & 4,450 & $81,061,295$ & 11,843 & 9,372 & $0.012 \%$ \\
\hline Mid-Continent & 284,000 & 5,241 & $91,603,941$ & 23,872 & 19,649 & $0.021 \%$ \\
\hline Northeast & 18,661 & 6,735 & $131,907,413$ & 24,523 & 23,041 & $0.017 \%$ \\
\hline Aggregate & $1,704,598$ & 20,147 & $367,669,378$ & 89,054 & 73,902 & $0.020 \%$ \\
\hline
\end{tabular}

MT: Metric Tonnes, Mbbl: Thousand barrels; GCF: Giga-Cubic Feet 
Table S31. Augmented facility-level data used to derive basin-level TNME GHGRP

\begin{tabular}{|c|c|c|c|c|c|c|c|c|c|}
\hline NEMS Region & Basin Name & Facility ID & $\begin{array}{l}\text { Saleable Oil } \\
\text { (Mbbl) }\end{array}$ & $\begin{array}{l}\text { Methane } \\
\text { Fraction } \\
(\%)\end{array}$ & $\begin{array}{l}\text { Natural Gas } \\
\text { Produced } \\
\text { (GCF) }\end{array}$ & $\begin{array}{l}\text { Methane Component } \\
\text { of Produced NG } \\
\left(\mathrm{MT} \mathrm{CH}_{4}\right)\end{array}$ & $\begin{array}{l}\text { Allocation } \\
\text { Factor (\%) }\end{array}$ & $\begin{array}{l}\text { Methane } \\
\text { Emissions, } \\
\text { Unallocated } \\
\left(\mathrm{MT} \mathrm{CH}_{4}\right)\end{array}$ & $\begin{array}{l}\text { Methane } \\
\text { Emissions, } \\
\text { Allocated } \\
\left(\mathrm{MT} \mathrm{CH}_{4}\right)\end{array}$ \\
\hline Mid-Continent & Anadarko Basin & 1008200 & 67 & $86 \%$ & 1 & 19,325 & $63 \%$ & 3 & 2 \\
\hline Mid-Continent & Anadarko Basin & 1008241 & 2,193 & $86 \%$ & 52 & 913,528 & $68 \%$ & 138 & 94 \\
\hline Mid-Continent & Anadarko Basin & 1008287 & 8,552 & $86 \%$ & 182 & $3,187,844$ & $67 \%$ & 547 & 366 \\
\hline Mid-Continent & Anadarko Basin & 1008306 & 955 & $86 \%$ & 110 & $1,914,684$ & $80 \%$ & 24 & 19 \\
\hline Mid-Continent & Anadarko Basin & 1008472 & 9,657 & $86 \%$ & 131 & $2,287,677$ & $60 \%$ & 9 & 5 \\
\hline Mid-Continent & Anadarko Basin & 1008530 & 9,337 & $86 \%$ & 138 & $2,412,134$ & $61 \%$ & 541 & 331 \\
\hline Mid-Continent & Anadarko Basin & 1008702 & 4,152 & $86 \%$ & 120 & $2,101,083$ & $71 \%$ & 59 & 42 \\
\hline Mid-Continent & Anadarko Basin & 1008703 & 9 & $86 \%$ & 2 & 36,532 & $82 \%$ & 59 & 48 \\
\hline Mid-Continent & Anadarko Basin & 1008956 & 287 & $86 \%$ & 9 & 162,438 & $72 \%$ & 0 & 0 \\
\hline Mid-Continent & Anadarko Basin & 1009135 & 100 & $86 \%$ & 16 & 283,539 & $81 \%$ & 84 & 68 \\
\hline Mid-Continent & Anadarko Basin & 1009143 & 1 & $86 \%$ & 49 & 848,495 & $84 \%$ & 11 & 10 \\
\hline Mid-Continent & Anadarko Basin & 1009260 & 6,244 & $86 \%$ & 53 & 922,836 & $51 \%$ & 7 & 4 \\
\hline Mid-Continent & Anadarko Basin & 1009335 & 3,842 & $86 \%$ & 85 & $1,486,025$ & $67 \%$ & 7 & 5 \\
\hline Mid-Continent & Anadarko Basin & 1012437 & 913 & $86 \%$ & 35 & 603,501 & $73 \%$ & 10 & 7 \\
\hline Mid-Continent & Anadarko Basin & 1008321 & 6 & $86 \%$ & 1 & 21,657 & $82 \%$ & 46 & 38 \\
\hline Mid-Continent & Anadarko Basin & 1008411 & 536 & $86 \%$ & 10 & 173,945 & $65 \%$ & 109 & 71 \\
\hline Mid-Continent & Anadarko Basin & 1008558 & 962 & $86 \%$ & 41 & 712,499 & $75 \%$ & 44 & 33 \\
\hline Mid-Continent & Anadarko Basin & 1008698 & 52 & $86 \%$ & 4 & 69,578 & $79 \%$ & 4 & 3 \\
\hline Mid-Continent & Anadarko Basin & 1009353 & 3,946 & $86 \%$ & 45 & 789,595 & $57 \%$ & 3 & 2 \\
\hline Mid-Continent & Anadarko Basin & 1009663 & 806 & $86 \%$ & 34 & 601,650 & $75 \%$ & 13 & 10 \\
\hline Mid-Continent & Anadarko Basin & 1010258 & 4,608 & $86 \%$ & 69 & $1,205,168$ & $61 \%$ & 71 & 43 \\
\hline Mid-Continent & Anadarko Basin & 1011854 & 4,449 & $86 \%$ & 108 & $1,888,206$ & $69 \%$ & 243 & 167 \\
\hline Mid-Continent & Anadarko Basin & 1008715 & 0 & $86 \%$ & 1 & 17,461 & $84 \%$ & 0 & 0 \\
\hline Mid-Continent & Anadarko Basin & 1008352 & 1,796 & $86 \%$ & 14 & 250,501 & $50 \%$ & 6 & 3 \\
\hline Mid-Continent & Anadarko Basin & 1011276 & 378 & $86 \%$ & 5 & 90,587 & $60 \%$ & 8 & 5 \\
\hline Northeast & Appalachian Basin (160) & 1008483 & 78 & $96 \%$ & 10 & 193,816 & $90 \%$ & 23 & 21 \\
\hline Northeast & Appalachian Basin (160) & 1008521 & 18 & $96 \%$ & 29 & 563,712 & $94 \%$ & 314 & 295 \\
\hline Northeast & Appalachian Basin (160) & 1008784 & 487 & $96 \%$ & 29 & 570,995 & $86 \%$ & 68 & 59 \\
\hline Northeast & Appalachian Basin (160) & 1009308 & 94 & $96 \%$ & 65 & $1,265,662$ & $94 \%$ & 142 & 133 \\
\hline Northeast & Appalachian Basin (160) & 1010718 & 1,230 & $96 \%$ & 86 & $1,679,593$ & $87 \%$ & 3 & 2 \\
\hline Northeast & Appalachian Basin (160) & 1008502 & 4 & $96 \%$ & 16 & 316,993 & $94 \%$ & 54 & 51 \\
\hline Northeast & Appalachian Basin (160) & 1008918 & 18 & $96 \%$ & 3 & 60,136 & $91 \%$ & 11 & 10 \\
\hline Northeast & Appalachian Basin $(160 \mathrm{~A})^{*}$ & 1008795 & - & $96 \%$ & 37 & 728,164 & $94 \%$ & 8 & 7 \\
\hline Northeast & Appalachian Basin (160A)* & 1006564 & 65 & $96 \%$ & 45 & 884,969 & $94 \%$ & 294 & 275 \\
\hline Northeast & Appalachian Basin (160A)* & 1008051 & 3,466 & $96 \%$ & 482 & $9,441,712$ & $91 \%$ & 637 & 578 \\
\hline Northeast & Appalachian Basin (160A)* & 1008116 & 0 & $96 \%$ & 587 & $11,501,782$ & $94 \%$ & 1,029 & 972 \\
\hline Northeast & Appalachian Basin (160A)* & 1008401 & 67 & $96 \%$ & 121 & $2,370,486$ & $94 \%$ & 498 & 469 \\
\hline
\end{tabular}




\begin{tabular}{|c|c|c|c|c|c|c|c|c|c|}
\hline Northeast & Appalachian Basin (160A)* & 1008480 & 110 & $96 \%$ & 33 & 648,916 & $93 \%$ & 1,315 & 1,220 \\
\hline Northeast & Appalachian Basin (160A)* & 1008481 & - & $96 \%$ & 196 & $3,843,208$ & $94 \%$ & 215 & 203 \\
\hline Northeast & Appalachian Basin $(160 \mathrm{~A})^{*}$ & 1008523 & 16 & $96 \%$ & 706 & $13,821,022$ & $94 \%$ & 745 & 703 \\
\hline Northeast & Appalachian Basin (160A)* & 1008524 & 7,207 & $96 \%$ & 332 & $6,508,328$ & $84 \%$ & 242 & 204 \\
\hline Northeast & Appalachian Basin $(160 \mathrm{~A})^{*}$ & 1008673 & - & $96 \%$ & 38 & 753,889 & $94 \%$ & 138 & 130 \\
\hline Northeast & Appalachian Basin (160A)* & 1008783 & 100 & $96 \%$ & 380 & $7,434,943$ & $94 \%$ & 12,748 & 12,022 \\
\hline Northeast & Appalachian Basin $(160 \mathrm{~A})^{*}$ & 1008882 & 76 & $96 \%$ & 52 & $1,010,523$ & $94 \%$ & 239 & 224 \\
\hline Northeast & Appalachian Basin $(160 \mathrm{~A})^{*}$ & 1010183 & 1,679 & $96 \%$ & 586 & $11,486,267$ & $93 \%$ & 419 & 389 \\
\hline Northeast & Appalachian Basin $(160 \mathrm{~A})^{*}$ & 1010233 & 818 & $96 \%$ & 829 & $16,229,806$ & $94 \%$ & 1,060 & 996 \\
\hline Northeast & Appalachian Basin $(160 \mathrm{~A})^{*}$ & 1011678 & - & $96 \%$ & 105 & $2,064,187$ & $94 \%$ & 7 & 7 \\
\hline Northeast & Appalachian Basin (160A)* & 1008503 & 0 & $96 \%$ & 686 & $13,431,995$ & $94 \%$ & 2,362 & 2,231 \\
\hline Northeast & Appalachian Basin $(160 \mathrm{~A})^{*}$ & 1009301 & 3 & $96 \%$ & 183 & $3,585,414$ & $94 \%$ & 479 & 452 \\
\hline Northeast & Appalachian Basin $(160 \mathrm{~A})^{*}$ & 1009732 & - & $96 \%$ & 103 & $2,017,039$ & $94 \%$ & 53 & 50 \\
\hline Northeast & Appalachian Basin $(160 \mathrm{~A})^{*}$ & 1008350 & - & $96 \%$ & 12 & 231,418 & $94 \%$ & 316 & 298 \\
\hline Northeast & Appalachian Basin $(160 \mathrm{~A})^{*}$ & 1008937 & - & $96 \%$ & 168 & $3,296,221$ & $94 \%$ & 552 & 521 \\
\hline Northeast & Appalachian Basin (160A)* & 1010721 & 3,124 & $96 \%$ & 545 & $10,668,033$ & $92 \%$ & 166 & 152 \\
\hline Northeast & Appalachian Basin (160A)* & 1011616 & - & $96 \%$ & 31 & 614,267 & $94 \%$ & 37 & 35 \\
\hline Northeast & Appalachian Basin $(160 \mathrm{~A})^{*}$ & 1011861 & - & $96 \%$ & 239 & $4,683,917$ & $94 \%$ & 349 & 330 \\
\hline Gulf Coast & Arkla Basin & 1012509 & 107 & $90 \%$ & 58 & $1,057,394$ & $87 \%$ & 5 & 5 \\
\hline Gulf Coast & Arkla Basin & 1008527 & 0 & $90 \%$ & 419 & $7,633,491$ & $88 \%$ & 651 & 572 \\
\hline Gulf Coast & Arkla Basin & 1009082 & 4 & $90 \%$ & 92 & $1,671,766$ & $88 \%$ & 23 & 20 \\
\hline Gulf Coast & Arkla Basin & 1009283 & 33 & $90 \%$ & 41 & 753,537 & $87 \%$ & 433 & 378 \\
\hline Gulf Coast & Arkla Basin & 1009686 & 128 & $90 \%$ & 13 & 240,363 & $83 \%$ & 207 & 173 \\
\hline Gulf Coast & Arkla Basin & 1008492 & 0 & $90 \%$ & 166 & $3,020,564$ & $88 \%$ & 106 & 93 \\
\hline Gulf Coast & Arkla Basin & 1011194 & 7 & $90 \%$ & 15 & 274,776 & $88 \%$ & 14 & 13 \\
\hline Gulf Coast & Arkla Basin & 1011913 & - & $90 \%$ & 59 & $1,078,168$ & $88 \%$ & 761 & 669 \\
\hline Gulf Coast & Arkla Basin & 1011998 & 1,896 & $90 \%$ & 178 & $3,244,548$ & $83 \%$ & 913 & 758 \\
\hline Gulf Coast & Arkla Basin & 1012018 & 17 & $90 \%$ & 6 & 106,316 & $86 \%$ & 2 & 1 \\
\hline Gulf Coast & Arkla Basin & 1008081 & - & $90 \%$ & 8 & 150,898 & $88 \%$ & 723 & 635 \\
\hline Gulf Coast & Arkla Basin & 1011848 & 34 & $90 \%$ & 10 & 185,185 & $86 \%$ & 1 & 1 \\
\hline Gulf Coast & Arkla Basin & 1012114 & 139 & $90 \%$ & 26 & 472,731 & $85 \%$ & 0 & 0 \\
\hline Gulf Coast & Arkla Basin & 1008640 & 124 & $90 \%$ & 1 & 22,488 & $56 \%$ & 31 & 18 \\
\hline Gulf Coast & Arkla Basin & 1008957 & - & $90 \%$ & 112 & $2,038,975$ & $88 \%$ & 247 & 217 \\
\hline Gulf Coast & Arkla Basin & 1008341 & 3 & $90 \%$ & 3 & 48,618 & $87 \%$ & 14 & 12 \\
\hline Gulf Coast & Arkla Basin & 1007599 & 371 & $90 \%$ & 67 & $1,211,387$ & $85 \%$ & 14 & 12 \\
\hline Gulf Coast & Arkla Basin & 1012774 & - & $90 \%$ & 33 & 598,766 & $88 \%$ & 1 & 1 \\
\hline Mid-Continent & Arkoma Basin & 1008199 & 0 & $86 \%$ & 11 & 199,306 & $84 \%$ & 84 & 71 \\
\hline Mid-Continent & Arkoma Basin & 1008474 & - & $86 \%$ & 18 & 319,992 & $84 \%$ & 628 & 529 \\
\hline Mid-Continent & Arkoma Basin & 1008588 & - & $86 \%$ & 89 & $1,552,412$ & $84 \%$ & 205 & 172 \\
\hline Mid-Continent & Arkoma Basin & 1009113 & 20 & $86 \%$ & 43 & 747,595 & $84 \%$ & 145 & 122 \\
\hline Mid-Continent & Arkoma Basin & 1009169 & 14 & $86 \%$ & 84 & $1,472,332$ & $84 \%$ & 418 & 352 \\
\hline Mid-Continent & Arkoma Basin & 1009310 & - & $86 \%$ & 9 & 149,393 & $84 \%$ & 668 & 563 \\
\hline Mid-Continent & Arkoma Basin & 1009392 & 0 & $86 \%$ & 454 & $7,927,537$ & $84 \%$ & 2,024 & 1,706 \\
\hline Mid-Continent & Arkoma Basin & 1009571 & 1 & $86 \%$ & 3 & 46,389 & $84 \%$ & 15 & 13 \\
\hline
\end{tabular}




\begin{tabular}{|c|c|c|c|c|c|c|c|c|c|}
\hline Mid-Continent & Arkoma Basin & 1010205 & - & $86 \%$ & 558 & $9,757,654$ & $84 \%$ & 15,103 & 12,728 \\
\hline Mid-Continent & Arkoma Basin & 1008753 & 24 & $86 \%$ & 3 & 46,345 & $80 \%$ & 221 & 177 \\
\hline Mid-Continent & Chautauqua Platform & 1009115 & 473 & $86 \%$ & 17 & 288,602 & $73 \%$ & 70 & 51 \\
\hline Mid-Continent & Chautauqua Platform & 1008286 & 15 & $86 \%$ & 0 & 5,628 & $67 \%$ & 1 & 1 \\
\hline Mid-Continent & Chautauqua Platform & 1008469 & 6 & $86 \%$ & 4 & 69,816 & $84 \%$ & 79 & 66 \\
\hline Mid-Continent & Chautauqua Platform & 1008244 & 186 & $86 \%$ & 0 & 4,538 & $17 \%$ & 0 & 0 \\
\hline Rocky Mountain & Denver Basin & 1008202 & 33,448 & $83 \%$ & 252 & $4,269,850$ & $47 \%$ & 75 & 35 \\
\hline Rocky Mountain & Denver Basin & 1008270 & 25,064 & $83 \%$ & 140 & $2,371,373$ & $41 \%$ & 290 & 119 \\
\hline Rocky Mountain & Denver Basin & 1008087 & 463 & $83 \%$ & 3 & 47,860 & $43 \%$ & 155 & 66 \\
\hline Rocky Mountain & Denver Basin & 1009286 & 11,093 & $83 \%$ & 83 & $1,399,392$ & $47 \%$ & 565 & 265 \\
\hline Rocky Mountain & Denver Basin & 1008794 & 2,778 & $83 \%$ & 13 & 215,221 & $37 \%$ & 26 & 9 \\
\hline Rocky Mountain & Denver Basin & 1008755 & 44 & $83 \%$ & 6 & 108,594 & $79 \%$ & 53 & 42 \\
\hline Rocky Mountain & Denver Basin & 1000355 & 4,624 & $83 \%$ & 20 & 342,945 & $36 \%$ & 97 & 35 \\
\hline Rocky Mountain & Denver Basin & 1011894 & 2,439 & $83 \%$ & 11 & 194,059 & $37 \%$ & 57 & 21 \\
\hline Rocky Mountain & Denver Basin & 1008206 & 5,249 & $83 \%$ & 53 & 900,997 & $53 \%$ & 2 & 1 \\
\hline Gulf Coast & East Texas Basin & 1008079 & 13 & $90 \%$ & 1,082 & $19,700,068$ & $88 \%$ & 3,359 & 2,950 \\
\hline Gulf Coast & East Texas Basin & 1009170 & 316 & $90 \%$ & 56 & $1,021,836$ & $85 \%$ & 19 & 16 \\
\hline Gulf Coast & East Texas Basin & 1009584 & 604 & $90 \%$ & 70 & $1,283,674$ & $84 \%$ & 238 & 200 \\
\hline Gulf Coast & East Texas Basin & 1010396 & 182 & $90 \%$ & 32 & 587,458 & $85 \%$ & 194 & 165 \\
\hline Gulf Coast & East Texas Basin & 1011976 & 121 & $90 \%$ & 6 & 102,319 & $78 \%$ & 1 & 1 \\
\hline Gulf Coast & East Texas Basin & 1010621 & 173 & $90 \%$ & 129 & $2,354,769$ & $87 \%$ & 38 & 33 \\
\hline Gulf Coast & East Texas Basin & 1008128 & 65 & $90 \%$ & 13 & 241,102 & $86 \%$ & 203 & 173 \\
\hline Gulf Coast & East Texas Basin & 1008587 & 346 & $90 \%$ & 27 & 491,294 & $82 \%$ & 1,191 & 977 \\
\hline Gulf Coast & East Texas Basin & 1008850 & 306 & $90 \%$ & 51 & 924,986 & $85 \%$ & 96 & 82 \\
\hline Gulf Coast & East Texas Basin & 1008958 & - & $90 \%$ & 64 & $1,158,195$ & $88 \%$ & 33 & 29 \\
\hline Gulf Coast & East Texas Basin & 1009239 & 67 & $90 \%$ & 5 & 95,749 & $82 \%$ & 0 & 0 \\
\hline Gulf Coast & East Texas Basin & 1011936 & 11 & $90 \%$ & 37 & 669,414 & $88 \%$ & 64 & 56 \\
\hline Gulf Coast & East Texas Basin & 1012782 & 23 & $90 \%$ & 6 & 111,063 & $86 \%$ & 474 & 407 \\
\hline Mid-Continent & Fort Worth Syncline & 1008289 & 467 & $86 \%$ & 273 & $4,779,904$ & $83 \%$ & 246 & 205 \\
\hline Mid-Continent & Fort Worth Syncline & 1008977 & 1,144 & $86 \%$ & 60 & $1,045,885$ & $76 \%$ & 7 & 5 \\
\hline Mid-Continent & Fort Worth Syncline & 1009388 & 0 & $86 \%$ & 55 & 967,037 & $84 \%$ & 60 & 50 \\
\hline Mid-Continent & Fort Worth Syncline & 1010237 & 192 & $86 \%$ & 8 & 145,832 & $75 \%$ & 22 & 16 \\
\hline Mid-Continent & Fort Worth Syncline & 1008356 & 71 & $86 \%$ & 11 & 189,948 & $81 \%$ & 12 & 9 \\
\hline Mid-Continent & Fort Worth Syncline & 1009441 & 4 & $86 \%$ & 17 & 289,628 & $84 \%$ & 25 & 21 \\
\hline Mid-Continent & Fort Worth Syncline & 1007476 & 118 & $86 \%$ & 22 & 382,485 & $82 \%$ & 2 & 1 \\
\hline Rocky Mountain & Green River Basin & 1008232 & 14 & $83 \%$ & 2 & 27,162 & $78 \%$ & 11 & 8 \\
\hline Rocky Mountain & Green River Basin & 1008590 & 192 & $83 \%$ & 44 & 743,471 & $80 \%$ & 420 & 335 \\
\hline Rocky Mountain & Green River Basin & 1008622 & 1,778 & $83 \%$ & 98 & $1,660,614$ & $74 \%$ & 521 & 387 \\
\hline Rocky Mountain & Green River Basin & 1008852 & 185 & $83 \%$ & 11 & 182,128 & $75 \%$ & 38 & 29 \\
\hline Rocky Mountain & Green River Basin & 1009258 & 11 & $83 \%$ & 2 & 35,473 & $80 \%$ & 211 & 168 \\
\hline Rocky Mountain & Green River Basin & 1010397 & 544 & $83 \%$ & 63 & $1,061,996$ & $78 \%$ & 429 & 335 \\
\hline Rocky Mountain & Green River Basin & 1012706 & 140 & $83 \%$ & 11 & 187,950 & $76 \%$ & 21 & 16 \\
\hline Rocky Mountain & Green River Basin & 1008295 & 1,460 & $83 \%$ & 194 & $3,286,592$ & $79 \%$ & 627 & 492 \\
\hline Rocky Mountain & Green River Basin & 1008954 & 152 & $83 \%$ & 267 & $4,536,670$ & $82 \%$ & 21 & 18 \\
\hline
\end{tabular}




\begin{tabular}{|c|c|c|c|c|c|c|c|c|c|}
\hline Rocky Mountain & Green River Basin & 1008204 & 24,271 & $83 \%$ & 7 & 122,937 & $4 \%$ & 23 & 1 \\
\hline Rocky Mountain & Green River Basin & 1008353 & 242 & $83 \%$ & 11 & 179,347 & $73 \%$ & 9 & 6 \\
\hline Rocky Mountain & Green River Basin & 1010157 & 30 & $83 \%$ & 6 & 107,628 & $80 \%$ & 801 & 639 \\
\hline Rocky Mountain & Green River Basin & 1011450 & 1,863 & $83 \%$ & 177 & $3,008,627$ & $77 \%$ & 129 & 100 \\
\hline Gulf Coast & Gulf Coast Basin & 1007485 & 45,711 & $90 \%$ & 171 & $3,112,780$ & $35 \%$ & 25 & 9 \\
\hline Gulf Coast & Gulf Coast Basin & 1008526 & 43,958 & $90 \%$ & 137 & $2,486,341$ & $32 \%$ & 250 & 79 \\
\hline Gulf Coast & Gulf Coast Basin & 1008604 & 178,442 & $90 \%$ & 101 & $1,836,856$ & $8 \%$ & 31 & 2 \\
\hline Gulf Coast & Gulf Coast Basin & 1009238 & 38,493 & $90 \%$ & 132 & $2,412,812$ & $34 \%$ & 377 & 127 \\
\hline Gulf Coast & Gulf Coast Basin & 1009706 & 1,024 & $90 \%$ & 78 & $1,423,583$ & $82 \%$ & 1 & 1 \\
\hline Gulf Coast & Gulf Coast Basin & 1010367 & 3,356 & $90 \%$ & 8 & 146,047 & $26 \%$ & 96 & 25 \\
\hline Gulf Coast & Gulf Coast Basin & 1009588 & 2,407 & $90 \%$ & 266 & $4,853,651$ & $84 \%$ & 142 & 119 \\
\hline Gulf Coast & Gulf Coast Basin & 1008219 & 82,674 & $90 \%$ & 154 & $2,809,420$ & $22 \%$ & 426 & 94 \\
\hline Gulf Coast & Gulf Coast Basin & 1008548 & 112 & $90 \%$ & 0 & 3,512 & $21 \%$ & 9 & 2 \\
\hline Gulf Coast & Gulf Coast Basin & 1008936 & 19,665 & $90 \%$ & 194 & $3,538,961$ & $56 \%$ & 165 & 93 \\
\hline Gulf Coast & Gulf Coast Basin & 1009386 & 7 & $90 \%$ & 45 & 828,199 & $88 \%$ & 25 & 22 \\
\hline Gulf Coast & Gulf Coast Basin & 1009511 & 2,126 & $90 \%$ & 5 & 83,396 & $25 \%$ & 30 & 7 \\
\hline Gulf Coast & Gulf Coast Basin & 1009624 & 2,615 & $90 \%$ & 178 & $3,237,034$ & $81 \%$ & 40 & 32 \\
\hline Gulf Coast & Gulf Coast Basin & 1011421 & 278 & $90 \%$ & 10 & 189,160 & $76 \%$ & 40 & 31 \\
\hline Gulf Coast & Gulf Coast Basin & 1008630 & 6,826 & $90 \%$ & 69 & $1,248,197$ & $57 \%$ & 16 & 9 \\
\hline Gulf Coast & Gulf Coast Basin & 1009609 & 185 & $90 \%$ & 9 & 167,053 & $79 \%$ & 3 & 2 \\
\hline Gulf Coast & Gulf Coast Basin & 1011275 & 1,156 & $90 \%$ & 7 & 132,395 & $47 \%$ & 110 & 51 \\
\hline Mid-Continent & Permian Basin & 1008141 & 85,345 & $86 \%$ & 203 & $3,545,169$ & $25 \%$ & 18 & 4 \\
\hline Mid-Continent & Permian Basin & 1008229 & 3,706 & $86 \%$ & 23 & 400,835 & $44 \%$ & 17 & 7 \\
\hline Mid-Continent & Permian Basin & 1008290 & 19,264 & $86 \%$ & 75 & $1,302,333$ & $35 \%$ & 28 & 10 \\
\hline Mid-Continent & Permian Basin & 1008449 & 15,593 & $86 \%$ & 80 & $1,404,301$ & $41 \%$ & 9 & 4 \\
\hline Mid-Continent & Permian Basin & 1008959 & 400 & $86 \%$ & 4 & 63,477 & $52 \%$ & 1 & 0 \\
\hline Mid-Continent & Permian Basin & 1009339 & 75 & $86 \%$ & 1 & 19,722 & $62 \%$ & 1 & 0 \\
\hline Mid-Continent & Permian Basin & 1009391 & 34,928 & $86 \%$ & 139 & $2,433,398$ & $35 \%$ & 1 & 0 \\
\hline Mid-Continent & Permian Basin & 1011662 & 344 & $86 \%$ & 7 & 116,859 & $66 \%$ & 12 & 8 \\
\hline Mid-Continent & Permian Basin & 1008351 & 22,605 & $86 \%$ & 83 & $1,449,792$ & $34 \%$ & 11 & 4 \\
\hline Mid-Continent & Permian Basin & 1009240 & 3,196 & $86 \%$ & 32 & 551,548 & $54 \%$ & 0 & 0 \\
\hline Mid-Continent & Permian Basin & 1010294 & 2,266 & $86 \%$ & 17 & 303,444 & $49 \%$ & 3 & 1 \\
\hline Mid-Continent & Permian Basin & 1009030 & 100 & $86 \%$ & 4 & 73,264 & $74 \%$ & 27 & 20 \\
\hline Mid-Continent & Permian Basin & 1011690 & 2,974 & $86 \%$ & 11 & 200,108 & $35 \%$ & 60 & 21 \\
\hline Mid-Continent & Permian Basin & 1008544 & 18,062 & $86 \%$ & 121 & $2,122,481$ & $46 \%$ & 18 & 8 \\
\hline Mid-Continent & Permian Basin & 1009273 & 1,271 & $86 \%$ & 3 & 57,455 & $27 \%$ & 6 & 2 \\
\hline Rocky Mountain & Piceance Basin & 1009785 & 143 & $83 \%$ & 50 & 841,226 & $80 \%$ & 475 & 382 \\
\hline Rocky Mountain & Piceance Basin & 1008296 & 41 & $83 \%$ & 32 & 540,360 & $81 \%$ & 216 & 176 \\
\hline Rocky Mountain & Piceance Basin & 1008323 & 10 & $83 \%$ & 2 & 25,649 & $79 \%$ & 47 & 37 \\
\hline Rocky Mountain & Piceance Basin & 1008355 & 611 & $83 \%$ & 245 & $4,149,706$ & $81 \%$ & 789 & 636 \\
\hline Rocky Mountain & Piceance Basin & 1009241 & 15 & $83 \%$ & 4 & 74,653 & $80 \%$ & 8 & 6 \\
\hline Rocky Mountain & Piceance Basin & 1008165 & 25 & $83 \%$ & 9 & 157,864 & $81 \%$ & 141 & 114 \\
\hline Rocky Mountain & Piceance Basin & 1008207 & 429 & $83 \%$ & 138 & $2,337,078$ & $80 \%$ & 28 & 22 \\
\hline Rocky Mountain & Piceance Basin & 1009141 & 2 & $83 \%$ & 356 & $6,046,034$ & $82 \%$ & 50 & 41 \\
\hline
\end{tabular}




\begin{tabular}{|c|c|c|c|c|c|c|c|c|c|}
\hline Rocky Mountain & Piceance Basin & 1011535 & 255 & $83 \%$ & 27 & 455,624 & $78 \%$ & 3 & 3 \\
\hline Rocky Mountain & Piceance Basin & 1010181 & 212 & $83 \%$ & 37 & 627,826 & $79 \%$ & 7 & 6 \\
\hline Rocky Mountain & San Juan Basin & 1005963 & 0 & $83 \%$ & 61 & $1,040,770$ & $82 \%$ & 10 & 8 \\
\hline Rocky Mountain & San Juan Basin & 1007484 & 739 & $83 \%$ & 356 & $6,036,006$ & $81 \%$ & 17,354 & 14,031 \\
\hline Rocky Mountain & San Juan Basin & 1008273 & 28 & $83 \%$ & 5 & 80,510 & $79 \%$ & 34 & 27 \\
\hline Rocky Mountain & San Juan Basin & 1008540 & 89 & $83 \%$ & 10 & 161,662 & $78 \%$ & 107 & 84 \\
\hline Rocky Mountain & San Juan Basin & 1008982 & 3,659 & $83 \%$ & 56 & 941,473 & $60 \%$ & 63 & 38 \\
\hline Rocky Mountain & San Juan Basin & 1009076 & 116 & $83 \%$ & 253 & $4,299,524$ & $82 \%$ & 143 & 116 \\
\hline Rocky Mountain & San Juan Basin & 1011100 & 28 & $83 \%$ & 6 & 108,419 & $80 \%$ & 31 & 25 \\
\hline Rocky Mountain & San Juan Basin & 1011978 & 8 & $83 \%$ & 47 & 793,128 & $82 \%$ & 139 & 113 \\
\hline Rocky Mountain & San Juan Basin & 1012724 & 424 & $83 \%$ & 13 & 215,592 & $69 \%$ & 281 & 194 \\
\hline Rocky Mountain & San Juan Basin & 1009142 & 0 & $83 \%$ & 154 & $2,611,485$ & $82 \%$ & 7 & 5 \\
\hline Rocky Mountain & San Juan Basin & 1009242 & 34 & $83 \%$ & 27 & 451,380 & $81 \%$ & - & - \\
\hline Mid-Continent & South Oklahoma Folded Belt & 1008383 & 743 & $86 \%$ & 20 & 347,881 & $70 \%$ & 12 & 8 \\
\hline Mid-Continent & South Oklahoma Folded Belt & 1008529 & 165 & $86 \%$ & 6 & 111,315 & $74 \%$ & 4 & 3 \\
\hline Mid-Continent & South Oklahoma Folded Belt & 1008616 & 15 & $86 \%$ & 250 & $4,367,242$ & $84 \%$ & 42 & 35 \\
\hline Mid-Continent & South Oklahoma Folded Belt & 1008545 & 108 & $86 \%$ & 3 & 48,161 & $69 \%$ & 5 & 4 \\
\hline Mid-Continent & South Oklahoma Folded Belt & 1008976 & 315 & $86 \%$ & 16 & 277,592 & $76 \%$ & 3 & 2 \\
\hline Mid-Continent & South Oklahoma Folded Belt & 1011191 & 3,165 & $86 \%$ & 79 & $1,379,816$ & $69 \%$ & 1 & 1 \\
\hline Mid-Continent & South Oklahoma Folded Belt & 1008134 & 2,742 & $86 \%$ & 82 & $1,441,801$ & $71 \%$ & 41 & 29 \\
\hline Mid-Continent & Strawn Basin & 1008188 & 6 & $86 \%$ & 28 & 480,854 & $84 \%$ & 38 & 32 \\
\hline Mid-Continent & Strawn Basin & 1008536 & 11 & $86 \%$ & 247 & $4,323,109$ & $84 \%$ & 206 & 174 \\
\hline Mid-Continent & Strawn Basin & 1009387 & 0 & $86 \%$ & 499 & $8,717,096$ & $84 \%$ & 931 & 785 \\
\hline Mid-Continent & Strawn Basin & 1009276 & 7 & $86 \%$ & 127 & $2,220,239$ & $84 \%$ & 229 & 193 \\
\hline Mid-Continent & Strawn Basin & 1010235 & 3 & $86 \%$ & 11 & 199,181 & $84 \%$ & 56 & 47 \\
\hline Mid-Continent & Strawn Basin & 1009666 & 6 & $86 \%$ & 15 & 256,723 & $84 \%$ & 13 & 11 \\
\hline Rocky Mountain & Uinta Basin & 1011596 & 77 & $83 \%$ & 22 & 372,542 & $80 \%$ & 504 & 405 \\
\hline Rocky Mountain & Uinta Basin & 1008354 & 475 & $83 \%$ & 26 & 445,520 & $74 \%$ & 94 & 70 \\
\hline Rocky Mountain & Uinta Basin & 1009165 & 807,359 & $83 \%$ & 160 & $2,706,673$ & $3 \%$ & 214 & 6 \\
\hline Rocky Mountain & Uinta Basin & 1009389 & 0 & $83 \%$ & 34 & 574,676 & $82 \%$ & 24 & 20 \\
\hline Rocky Mountain & Uinta Basin & 1012013 & 839 & $83 \%$ & 10 & 165,781 & $55 \%$ & 1,741 & 964 \\
\hline Rocky Mountain & Uinta Basin & 1009282 & 903 & $83 \%$ & 30 & 509,719 & $70 \%$ & 1,395 & 978 \\
\hline Rocky Mountain & Williston Basin & 1008471 & 34,078 & $83 \%$ & 64 & $1,088,529$ & $21 \%$ & 0 & 0 \\
\hline Rocky Mountain & Williston Basin & 1012100 & 712 & $83 \%$ & 13 & 220,412 & $63 \%$ & 322 & 202 \\
\hline Rocky Mountain & Williston Basin & 1011463 & 421 & $83 \%$ & 2 & 26,022 & $32 \%$ & 5 & 2 \\
\hline
\end{tabular}

*Appalachian Basin (160A): Appalachian Eastern Overthrust

MT: Metric Tonnes, Mbbl: Thousand barrels; GCF: Giga-Cubic Feet 


\section{S12. Comparison with the 2016 GHGRP}

Differences in absolute emissions between this work and the GHGRP can be attributed to: (1) technological disaggregation of plunger-lifts into manual-plunger lift and automatic plunger-lift systems, (2) estimated venting well count for manual and automatic plunger-lift systems, and (3) parameter distributions used to characterize natural gas liquids unloading, (4) data inputs and data coverage, and (5) deterministic vs stochastic modeling. Detailed basin-level comparisons between the current work and the 2016 GHGRP are provided in Table S32 through Table S49. 
Table S32. Benchmarking liquids unloading activities in the Anadarko basin, comparison between the 2016 GHGRP and NETL (this work)

\begin{tabular}{|c|c|c|c|c|c|c|c|c|c|c|c|c|c|}
\hline & \multicolumn{5}{|c|}{2016 GHGRP } & \multicolumn{5}{|c|}{ NETL (Stochastic) } & \multicolumn{3}{|c|}{ NETL (Deterministic) } \\
\hline Anadarko Basin & $\begin{array}{l}\text { Venting } \\
\text { Wells (\#) }\end{array}$ & $\begin{array}{l}\text { Venting } \\
\text { Events } \\
\text { (\#) }\end{array}$ & $\begin{array}{l}\text { *Methane } \\
\text { Emissions } \\
\left(\mathrm{MT} \mathrm{CH}_{4}\right)\end{array}$ & $\begin{array}{l}\text { *Methane } \\
\text { Emissions Per } \\
\text { Venting Event } \\
\text { (MT CH} / \text { / event) }\end{array}$ & $\begin{array}{l}\text { *Methane } \\
\text { Emissions Per } \\
\text { Venting Well } \\
\left(\mathrm{MT} \mathrm{CH}_{4} /\right. \\
\text { venting-well) }\end{array}$ & $\begin{array}{l}\text { Venting } \\
\text { Wells (\#) }\end{array}$ & $\begin{array}{l}\text { Venting } \\
\text { Events } \\
\text { (\#) }\end{array}$ & $\begin{array}{l}\text { *Methane } \\
\text { Emissions } \\
\left(\mathrm{MT} \mathrm{CH}_{4}\right)\end{array}$ & $\begin{array}{l}\text { *Methane } \\
\text { Emissions Per } \\
\text { Venting Event } \\
\left(\mathrm{MT} \mathrm{CH}_{4} / \text { event) }\right.\end{array}$ & $\begin{array}{l}\text { *Methane } \\
\text { Emissions Per } \\
\text { Venting Well } \\
\left(\mathrm{MT} \mathrm{CH}_{4} /\right. \\
\text { venting-well) }\end{array}$ & $\begin{array}{l}\text { Venting } \\
\text { Wells (\#) }\end{array}$ & $\begin{array}{l}\text { Venting } \\
\text { Events } \\
\text { (\#) }\end{array}$ & $\begin{array}{l}\text { *Methane } \\
\text { Emissions } \\
\left(\mathrm{MT} \mathrm{CH}_{4}\right)\end{array}$ \\
\hline $\begin{array}{l}\text { Conventional, } \\
\text { Non-Plunger }\end{array}$ & 259 & 6,061 & 884 & 0.15 & 3.41 & 411 & 15,885 & 2,139 & 0.13 & 5.20 & 411 & 15,902 & 1,938 \\
\hline $\begin{array}{l}\text { Conventional, } \\
\text { Plunger-Lift }\end{array}$ & 1,193 & 22,495 & 738 & 0.03 & 0.62 & 1,625 & 84,229 & 3,828 & 0.05 & 2.36 & 1,625 & 39,437 & 1,773 \\
\hline $\begin{array}{l}\text { Unconventional, } \\
\text { Non-Plunger }\end{array}$ & 98 & 367 & 283 & 0.77 & 2.89 & 415 & 16,033 & 6,682 & 0.40 & 16.10 & 415 & 16,041 & 5,859 \\
\hline $\begin{array}{l}\text { Unconventional, } \\
\text { Plunger-Lift }\end{array}$ & 101 & 472 & 142 & 0.30 & 1.41 & 516 & 8,197 & 709 & 0.09 & 1.37 & 516 & 7,222 & 419 \\
\hline Total & 1,651 & 29,395 & 2,048 & 0.07 & 1.24 & 2,967 & 124,344 & 13,358 & 0.11 & 4.50 & 2,967 & 78,602 & 9,989 \\
\hline
\end{tabular}

*Unallocated methane emissions from liquids unloading

Table S33. Benchmarking liquids unloading activities in the Appalachian Eastern Overthrust basin, comparison between the 2016 GHGRP and NETL (this work)

\begin{tabular}{|c|c|c|c|c|c|c|c|c|c|c|c|c|c|}
\hline & \multicolumn{5}{|c|}{2016 GHGRP } & \multicolumn{5}{|c|}{ NETL (Stochastic) } & \multicolumn{3}{|c|}{ NETL (Deterministic) } \\
\hline $\begin{array}{l}\text { Appalachian } \\
\text { Eastern } \\
\text { Overthrust }\end{array}$ & $\begin{array}{l}\text { Venting } \\
\text { Wells (\#) }\end{array}$ & $\begin{array}{l}\text { Venting } \\
\text { Events } \\
\text { (\#) }\end{array}$ & $\begin{array}{l}\text { *Methane } \\
\text { Emissions } \\
\left(\mathrm{MT} \mathrm{CH}_{4}\right)\end{array}$ & $\begin{array}{l}\text { *Methane } \\
\text { Emissions Per } \\
\text { Venting Event } \\
\text { (MT CH } \mathrm{CH}_{4} / \text { event) }\end{array}$ & $\begin{array}{l}\text { *Methane } \\
\text { Emissions Per } \\
\text { Venting Well (MT } \\
\mathrm{CH}_{4} / \text { venting-well) }\end{array}$ & $\begin{array}{l}\text { Venting } \\
\text { Wells (\#) }\end{array}$ & $\begin{array}{l}\text { Venting } \\
\text { Events } \\
\text { (\#) }\end{array}$ & $\begin{array}{l}\text { *Methane } \\
\text { Emissions } \\
\left(\mathrm{MT} \mathrm{CH}_{4}\right)\end{array}$ & $\begin{array}{l}\text { *Methane } \\
\text { Emissions Per } \\
\text { Venting Event } \\
\text { (MT CH }{ }_{4} / \text { event) }\end{array}$ & $\begin{array}{l}\text { *Methane } \\
\text { Emissions Per } \\
\text { Venting Well (MT } \\
\mathrm{CH}_{4} / \text { venting-well) }\end{array}$ & $\begin{array}{l}\text { Venting } \\
\text { Wells (\#) }\end{array}$ & $\begin{array}{l}\text { Venting } \\
\text { Events } \\
\text { (\#) }\end{array}$ & $\begin{array}{l}\text { *Methane } \\
\text { Emissions } \\
\left(\mathrm{MT} \mathrm{CH}_{4}\right)\end{array}$ \\
\hline $\begin{array}{l}\text { Conventional, } \\
\text { Non-Plunger }\end{array}$ & 1,548 & 26,809 & 8,384 & 0.31 & 5.42 & 3,648 & 140,952 & 7,943 & 0.06 & 2.18 & 3,648 & 141,038 & 7,578 \\
\hline $\begin{array}{l}\text { Conventional, } \\
\text { Plunger-Lift }\end{array}$ & 4,041 & 94,197 & 3,348 & 0.04 & 0.83 & 7,454 & 437,839 & 6,427 & 0.01 & 0.86 & 7,454 & 215,059 & 2,995 \\
\hline $\begin{array}{l}\text { Unconventional, } \\
\text { Non-Plunger }\end{array}$ & 1,554 & 8,878 & 9,903 & 1.12 & 6.37 & 1,651 & 63,674 & 69,635 & 1.04 & 42.18 & 1,651 & 63,825 & 49,634 \\
\hline $\begin{array}{l}\text { Unconventional, } \\
\text { Plunger-Lift }\end{array}$ & 1,133 & 18,508 & 2,271 & 0.12 & 2.00 & 1,359 & 32,261 & 9,052 & 0.29 & 6.66 & 1,359 & 21,504 & 4,179 \\
\hline Total & 8,276 & 148,392 & 23,907 & 0.16 & 2.89 & 14,112 & 674,726 & $\mathbf{9 3 , 0 5 7}$ & 0.15 & 6.59 & 14,112 & 441,427 & 64,387 \\
\hline
\end{tabular}


Table S34. Benchmarking liquids unloading activities in the Appalachian basin, comparison between the 2016 GHGRP and NETL (this work)

\begin{tabular}{|c|c|c|c|c|c|c|c|c|c|c|c|c|c|}
\hline & \multicolumn{5}{|c|}{2016 GHGRP } & \multicolumn{5}{|c|}{ NETL (Stochastic) } & \multicolumn{3}{|c|}{ NETL (Deterministic) } \\
\hline $\begin{array}{l}\text { Appalachian } \\
\text { Basin }\end{array}$ & $\begin{array}{l}\text { Venting } \\
\text { Wells (\#) }\end{array}$ & $\begin{array}{l}\text { Venting } \\
\text { Events } \\
\text { (\#) }\end{array}$ & $\begin{array}{l}\text { *Methane } \\
\text { Emissions } \\
\left(\mathrm{MT} \mathrm{CH}_{4}\right)\end{array}$ & $\begin{array}{l}\text { *Methane } \\
\text { Emissions Per } \\
\text { Venting Event } \\
\left(\mathrm{MT} \mathrm{CH}_{4} / \text { event }\right)\end{array}$ & $\begin{array}{l}\text { *Methane } \\
\text { Emissions Per } \\
\text { Venting Well (MT } \\
\mathrm{CH}_{4} / \text { venting-well) }\end{array}$ & $\begin{array}{l}\text { Venting } \\
\text { Wells (\#) }\end{array}$ & $\begin{array}{l}\text { Venting } \\
\text { Events } \\
(\#)\end{array}$ & $\begin{array}{l}\text { *Methane } \\
\text { Emissions } \\
\left(\mathrm{MT} \mathrm{CH}_{4}\right)\end{array}$ & $\begin{array}{l}\text { *Methane } \\
\text { Emissions Per } \\
\text { Venting Event } \\
\left(\mathrm{MT} \mathrm{CH}_{4} / \text { event }\right)\end{array}$ & $\begin{array}{l}\text { *Methane } \\
\text { Emissions Per } \\
\text { Venting Well (MT } \\
\mathrm{CH}_{4} / \text { venting-well) }\end{array}$ & $\begin{array}{l}\text { Venting } \\
\text { Wells (\#) }\end{array}$ & $\begin{array}{l}\text { Venting } \\
\text { Events } \\
\text { (\#) }\end{array}$ & $\begin{array}{l}\text { *Methane } \\
\text { Emissions } \\
\left(\mathrm{MT} \mathrm{CH}_{4}\right)\end{array}$ \\
\hline $\begin{array}{l}\text { Conventional, } \\
\text { Non-Plunger }\end{array}$ & 313 & 6,543 & 144 & 0.02 & 0.46 & 494 & 19,102 & 1,215 & 0.06 & 2.46 & 494 & 19,099 & 1,153 \\
\hline $\begin{array}{l}\text { Conventional, } \\
\text { Plunger-Lift }\end{array}$ & 1,624 & 20,155 & 91 & 0.00 & 0.06 & 3,119 & 88,743 & 850 & 0.01 & 0.27 & 3,119 & 53,776 & 485 \\
\hline $\begin{array}{l}\text { Unconventional, } \\
\text { Non-Plunger }\end{array}$ & 526 & 3,865 & 140 & 0.04 & 0.27 & 39 & 1,500 & 294 & 0.20 & 7.57 & 39 & 1,501 & 241 \\
\hline $\begin{array}{l}\text { Unconventional, } \\
\text { Plunger-Lift }\end{array}$ & 653 & 52,447 & 242 & 0.00 & 0.37 & 98 & 15,365 & 589 & 0.04 & 6.02 & 98 & 7,454 & 279 \\
\hline Total & 3,116 & 83,010 & 616 & 0.01 & 0.20 & 3,750 & 124,710 & 2,949 & 0.02 & 0.79 & 3,750 & 81,830 & 2,159 \\
\hline
\end{tabular}

*Unallocated methane emissions from liquids unloading

Table S35. Benchmarking liquids unloading activities in the Arkla basin, comparison between the 2016 GHGRP and NETL (this work)

\begin{tabular}{|c|c|c|c|c|c|c|c|c|c|c|c|c|c|}
\hline \multirow[b]{2}{*}{ Arkla Basin } & \multicolumn{5}{|c|}{2016 GHGRP } & \multicolumn{5}{|c|}{ NETL (Stochastic) } & \multicolumn{3}{|c|}{ NETL (Deterministic) } \\
\hline & $\begin{array}{l}\text { Venting } \\
\text { Wells (\#) }\end{array}$ & $\begin{array}{l}\text { Venting } \\
\text { Events } \\
(\#)\end{array}$ & $\begin{array}{l}\text { *Methane } \\
\text { Emissions } \\
\left(\mathrm{MT} \mathrm{CH}_{4}\right)\end{array}$ & $\begin{array}{l}\text { Methane } \\
\text { Emissions Per } \\
\text { Venting Event } \\
\left(\mathrm{MT} \mathrm{CH}_{4} / \text { event) }\right.\end{array}$ & $\begin{array}{l}\text { *Methane } \\
\text { Emissions Per } \\
\text { Venting Well (MT } \\
\mathrm{CH}_{4} / \text { venting-well) }\end{array}$ & $\begin{array}{l}\text { Venting } \\
\text { Wells (\#) }\end{array}$ & $\begin{array}{l}\text { Venting } \\
\text { Events } \\
(\#)\end{array}$ & $\begin{array}{l}\text { *Methane } \\
\text { Emissions } \\
\left(\mathrm{MT} \mathrm{CH}_{4}\right)\end{array}$ & $\begin{array}{l}\text { *Methane } \\
\text { Emissions Per } \\
\text { Venting Event } \\
\left(\mathrm{MT} \mathrm{CH}_{4} / \text { event) }\right.\end{array}$ & $\begin{array}{l}\text { *Methane } \\
\text { Emissions Per } \\
\text { Venting Well (MT } \\
\mathrm{CH}_{4} / \text { venting-well) }\end{array}$ & $\begin{array}{l}\text { Venting } \\
\text { Wells (\#) }\end{array}$ & $\begin{array}{l}\text { Venting } \\
\text { Events } \\
\text { (\#) }\end{array}$ & $\begin{array}{l}\text { *Methane } \\
\text { Emissions } \\
\left(\mathrm{MT} \mathrm{CH}_{4}\right)\end{array}$ \\
\hline $\begin{array}{l}\text { Conventional, } \\
\text { Non-Plunger }\end{array}$ & 1,133 & 6,249 & 1,267 & 0.20 & 1.12 & 2,521 & 97,920 & 16,641 & 0.17 & 6.60 & 2,521 & 97,468 & 15,746 \\
\hline $\begin{array}{l}\text { Conventional, } \\
\text { Plunger-Lift }\end{array}$ & 94 & 100,538 & 36 & 0.00 & 0.38 & 225 & 284,593 & 10,941 & 0.04 & 48.65 & 225 & 274,514 & 10,301 \\
\hline $\begin{array}{l}\text { Unconventional, } \\
\text { Non-Plunger }\end{array}$ & 545 & 4,891 & 2,814 & 0.58 & 5.16 & 802 & 31,078 & 30,400 & 0.93 & 37.91 & 802 & 31,004 & 23,926 \\
\hline $\begin{array}{l}\text { Unconventional, } \\
\text { Plunger-Lift }\end{array}$ & 24 & 108 & 31 & 0.28 & 1.28 & 39 & 580 & 157 & 0.28 & 4.06 & 39 & 540 & 90 \\
\hline Total & 1,796 & 111,786 & 4,147 & 0.04 & 2.31 & $3, \mathbf{5 8 7}$ & 414,172 & 58,139 & 0.15 & 16.21 & $\mathbf{3 , 5 8 7}$ & 403,527 & 50,063 \\
\hline
\end{tabular}

*Unallocated methane emissions from liquids unloading 
Table S36. Benchmarking liquids unloading activities in the Arkoma basin, comparison between the 2016 GHGRP and NETL (this work)

\begin{tabular}{|c|c|c|c|c|c|c|c|c|c|c|c|c|c|}
\hline & \multicolumn{5}{|c|}{2016 GHGRP } & \multicolumn{5}{|c|}{ NETL (Stochastic) } & \multicolumn{3}{|c|}{ NETL (Deterministic) } \\
\hline Arkoma Basin & $\begin{array}{l}\text { Venting } \\
\text { Wells (\#) }\end{array}$ & $\begin{array}{l}\text { Venting } \\
\text { Events } \\
(\#)\end{array}$ & $\begin{array}{l}\text { *Methane } \\
\text { Emissions } \\
\left(\mathrm{MT} \mathrm{CH}_{4}\right)\end{array}$ & $\begin{array}{l}\text { *Methane } \\
\text { Emissions Per } \\
\text { Venting Event } \\
\text { (MT CH } / \text { event) }\end{array}$ & $\begin{array}{l}\text { Methane } \\
\text { Emissions Per } \\
\text { Venting Well (MT } \\
\mathrm{CH}_{4} / \text { venting-well) }\end{array}$ & $\begin{array}{l}\text { Venting } \\
\text { Wells (\#) }\end{array}$ & $\begin{array}{l}\text { Venting } \\
\text { Events } \\
(\#)\end{array}$ & $\begin{array}{l}\text { *Methane } \\
\text { Emissions } \\
\left(\mathrm{MT} \mathrm{CH}_{4}\right)\end{array}$ & $\begin{array}{l}\text { *Methane } \\
\text { Emissions Per } \\
\text { Venting Event } \\
\text { (MT CH }{ }_{4} / \text { event) }\end{array}$ & $\begin{array}{l}\text { *Methane } \\
\text { Emissions Per } \\
\text { Venting Well (MT } \\
\mathrm{CH}_{4} / \text { venting-well) }\end{array}$ & $\begin{array}{l}\text { Venting } \\
\text { Wells (\#) }\end{array}$ & $\begin{array}{l}\text { Venting } \\
\text { Events } \\
\text { (\#) }\end{array}$ & $\begin{array}{l}\text { *Methane } \\
\text { Emissions } \\
\left(\mathrm{MT} \mathrm{CH}_{4}\right)\end{array}$ \\
\hline $\begin{array}{l}\text { Conventional, } \\
\text { Non-Plunger }\end{array}$ & 2,069 & 14,517 & 11,160 & 0.77 & 5.39 & 1,126 & 43,818 & 3,754 & 0.09 & 3.33 & 1,126 & 43,527 & 3,455 \\
\hline $\begin{array}{l}\text { Conventional, } \\
\text { Plunger-Lift }\end{array}$ & 709 & 598,041 & 5,643 & 0.01 & 7.96 & 1,376 & 410,343 & 24,063 & 0.06 & 17.48 & 1,376 & 329,312 & 19,090 \\
\hline $\begin{array}{l}\text { Unconventional, } \\
\text { Non-Plunger }\end{array}$ & 385 & 1,543 & 1,201 & 0.78 & 3.12 & 1,774 & 68,873 & 26,246 & 0.38 & 14.80 & 1,774 & 68,578 & 22,097 \\
\hline $\begin{array}{l}\text { Unconventional, } \\
\text { Plunger-Lift }\end{array}$ & 388 & 77,697 & 1,506 & 0.02 & 3.88 & 1,635 & 727,257 & 14,302 & 0.02 & 8.75 & 1,635 & 513,030 & 9,596 \\
\hline Total & 3,551 & 691,798 & 19,510 & 0.03 & 5.49 & 5,911 & $1,250,291$ & 68,365 & 0.06 & 11.57 & 5,911 & 954,447 & 54,238 \\
\hline
\end{tabular}

*Unallocated methane emissions from liquids unloading

Table S37. Benchmarking liquids unloading activities in the Chautauqua basin, comparison between the 2016 GHGRP and NETL (this work)

\begin{tabular}{|c|c|c|c|c|c|c|c|c|c|c|c|c|c|}
\hline & \multicolumn{5}{|c|}{2016 GHGRP } & \multicolumn{5}{|c|}{ NETL (Stochastic) } & \multicolumn{3}{|c|}{ NETL (Deterministic) } \\
\hline $\begin{array}{l}\text { Chautauqua } \\
\text { Basin }\end{array}$ & $\begin{array}{l}\text { Venting } \\
\text { Wells (\#) }\end{array}$ & $\begin{array}{l}\text { Venting } \\
\text { Events } \\
\text { (\#) }\end{array}$ & $\begin{array}{l}\text { *Methane } \\
\text { Emissions } \\
\left(\mathrm{MT} \mathrm{CH}_{4}\right)\end{array}$ & $\begin{array}{l}\text { *Methane } \\
\text { Emissions Per } \\
\text { Venting Event } \\
\text { (MT CH} / \text { event) }\end{array}$ & $\begin{array}{l}\text { *Methane } \\
\text { Emissions Per } \\
\text { Venting Well (MT } \\
\mathrm{CH}_{4} / \text { venting-well) }\end{array}$ & $\begin{array}{l}\text { Venting } \\
\text { Wells (\#) }\end{array}$ & $\begin{array}{l}\text { Venting } \\
\text { Events } \\
\text { (\#) }\end{array}$ & $\begin{array}{l}* \text { Methane } \\
\text { Emissions } \\
\left(\mathrm{MT} \mathrm{CH}_{4}\right)\end{array}$ & $\begin{array}{l}\text { *Methane } \\
\text { Emissions Per } \\
\text { Venting Event } \\
\text { (MT CH} / \text { event) }\end{array}$ & $\begin{array}{l}\text { *Methane } \\
\text { Emissions Per } \\
\text { Venting Well (MT } \\
\mathrm{CH}_{4} / \text { venting-well) }\end{array}$ & $\begin{array}{l}\text { Venting } \\
\text { Wells (\#) }\end{array}$ & $\begin{array}{l}\text { Venting } \\
\text { Events } \\
\text { (\#) }\end{array}$ & $\begin{array}{l}\text { *Methane } \\
\text { Emissions } \\
\left(\mathrm{MT} \mathrm{CH}_{4}\right)\end{array}$ \\
\hline $\begin{array}{l}\text { Conventional, } \\
\text { Non-Plunger }\end{array}$ & 2 & 3 & 1 & 0.43 & 0.64 & 101 & 3,908 & 225 & 0.05 & 2.23 & 101 & 3,903 & 210 \\
\hline $\begin{array}{l}\text { Conventional, } \\
\text { Plunger-Lift }\end{array}$ & 2 & 2 & 0 & 0.03 & 0.03 & 85 & 1,216 & 41 & 0.03 & 0.48 & 85 & 1,195 & 37 \\
\hline $\begin{array}{l}\text { Unconventional, } \\
\text { Non-Plunger }\end{array}$ & 42 & 134 & 81 & 0.60 & 1.92 & 243 & 9,403 & 1,225 & 0.14 & 5.05 & 243 & 9,379 & 1,118 \\
\hline $\begin{array}{l}\text { Unconventional, } \\
\text { Plunger-Lift }\end{array}$ & 21 & 33 & 68 & 2.05 & 3.23 & 121 & 1,713 & 43 & 0.03 & 0.36 & 121 & 1,690 & 29 \\
\hline Total & 67 & 172 & 150 & 0.87 & 2.23 & 550 & 16,240 & 1,534 & 0.09 & 2.79 & 550 & 16,167 & 1,394 \\
\hline
\end{tabular}

*Unallocated methane emissions from liquids unloading 
Table S38. Benchmarking liquids unloading activities in the Denver basin, comparison between the 2016 GHGRP and NETL (this work)

\begin{tabular}{|c|c|c|c|c|c|c|c|c|c|c|c|c|c|}
\hline & \multicolumn{5}{|c|}{2016 GHGRP } & \multicolumn{5}{|c|}{ NETL (Stochastic) } & \multicolumn{3}{|c|}{ NETL (Deterministic) } \\
\hline Denver Basin & $\begin{array}{l}\text { Venting } \\
\text { Wells (\#) }\end{array}$ & $\begin{array}{l}\text { Venting } \\
\text { Events } \\
\text { (\#) }\end{array}$ & $\begin{array}{l}\text { *Methane } \\
\text { Emissions } \\
\left(\mathrm{MT} \mathrm{CH}_{4}\right)\end{array}$ & $\begin{array}{l}\text { Methane } \\
\text { Emissions Per } \\
\text { Venting Event } \\
\left(\text { MT CH } \text { CHevent }_{4} \text { ) }\right.\end{array}$ & $\begin{array}{l}\text { *Methane } \\
\text { Emissions Per } \\
\text { Venting Well (MT } \\
\mathrm{CH}_{4} / \text { venting-well) }\end{array}$ & $\begin{array}{l}\text { Venting } \\
\text { Wells (\#) }\end{array}$ & $\begin{array}{l}\text { Venting } \\
\text { Events } \\
(\#)\end{array}$ & $\begin{array}{l}\text { *Methane } \\
\text { Emissions } \\
\left(\mathrm{MT} \mathrm{CH}_{4}\right)\end{array}$ & $\begin{array}{l}\text { *Methane } \\
\text { Emissions Per } \\
\text { Venting Event } \\
\text { (MT CH }{ }_{4} / \text { event) }\end{array}$ & $\begin{array}{l}\text { *Methane } \\
\text { Emissions Per } \\
\text { Venting Well (MT } \\
\mathrm{CH}_{4} / \text { venting-well) }\end{array}$ & $\begin{array}{l}\text { Venting } \\
\text { Wells (\#) }\end{array}$ & $\begin{array}{l}\text { Venting } \\
\text { Events } \\
\text { (\#) }\end{array}$ & $\begin{array}{l}\text { *Methane } \\
\text { Emissions } \\
\left(\mathrm{MT} \mathrm{CH}_{4}\right)\end{array}$ \\
\hline $\begin{array}{l}\text { Conventional, } \\
\text { Non-Plunger }\end{array}$ & 174 & 727 & 271 & 0.37 & 1.56 & 56 & 2,139 & 188 & 0.08 & 3.39 & 56 & 2,149 & 184 \\
\hline $\begin{array}{l}\text { Conventional, } \\
\text { Plunger-Lift }\end{array}$ & 2,354 & 11,595 & 1,047 & 0.09 & 0.44 & 638 & 11,127 & 589 & 0.05 & 0.92 & 638 & 8,921 & 446 \\
\hline $\begin{array}{l}\text { Unconventional, } \\
\text { Non-Plunger }\end{array}$ & 4 & 4 & 2 & 0.45 & 0.45 & 27 & 1,023 & 377 & 0.34 & 14.20 & 27 & 1,027 & 369 \\
\hline $\begin{array}{l}\text { Unconventional, } \\
\text { Plunger-Lift }\end{array}$ & - & - & - & - & - & - & - & - & - & - & - & - & - \\
\hline Total & 2,532 & 12,326 & 1,320 & 0.11 & 0.52 & 720 & 14,290 & 1,154 & 0.09 & 1.60 & 720 & 12,096 & 999 \\
\hline
\end{tabular}

*Unallocated methane emissions from liquids unloading

Table S39. Benchmarking liquids unloading activities in the East Texas basin, comparison between the 2016 GHGRP and NETL (this work)

\begin{tabular}{|c|c|c|c|c|c|c|c|c|c|c|c|c|c|}
\hline & \multicolumn{5}{|c|}{2016 GHGRP } & \multicolumn{5}{|c|}{ NETL (Stochastic) } & \multicolumn{3}{|c|}{ NETL (Deterministic) } \\
\hline East Texas Basin & $\begin{array}{l}\text { Venting } \\
\text { Wells (\#) }\end{array}$ & $\begin{array}{l}\text { Venting } \\
\text { Events } \\
\text { (\#) }\end{array}$ & $\begin{array}{l}\text { *Methane } \\
\text { Emissions } \\
\left(\mathrm{MT} \mathrm{CH}_{4}\right)\end{array}$ & $\begin{array}{l}\text { *Methane } \\
\text { Emissions Per } \\
\text { Venting Event } \\
\text { (MT CH} 4 \text { event) }\end{array}$ & $\begin{array}{l}\text { *Methane } \\
\text { Emissions Per } \\
\text { Venting Well (MT } \\
\mathrm{CH}_{4} / \text { venting-well) }\end{array}$ & $\begin{array}{l}\text { Venting } \\
\text { Wells (\#) }\end{array}$ & $\begin{array}{l}\text { Venting } \\
\text { Events } \\
\text { (\#) }\end{array}$ & $\begin{array}{l}\text { *Methane } \\
\text { Emissions } \\
\left(\mathrm{MT} \mathrm{CH}_{4}\right)\end{array}$ & $\begin{array}{l}\text { *Methane } \\
\text { Emissions Per } \\
\text { Venting Event } \\
\text { (MT CH} \mathrm{CH}_{4} / \text { event) }\end{array}$ & $\begin{array}{l}\text { *Methane } \\
\text { Emissions Per } \\
\text { Venting Well (MT } \\
\mathrm{CH}_{4} / \text { venting-well) }\end{array}$ & $\begin{array}{l}\text { Venting } \\
\text { Wells } \\
\text { (\#) }\end{array}$ & $\begin{array}{l}\text { Venting } \\
\text { Events } \\
(\#)\end{array}$ & $\begin{array}{l}\text { *Methane Emissions } \\
\left(\mathrm{MT} \mathrm{CH}_{4}\right)\end{array}$ \\
\hline $\begin{array}{l}\text { Conventional, } \\
\text { Non-Plunger }\end{array}$ & 794 & 3,820 & 5,219 & 1.37 & 6.57 & 872 & 33,715 & 12,244 & 0.35 & 14.03 & 872 & 33,729 & 11,496 \\
\hline $\begin{array}{l}\text { Conventional, } \\
\text { Plunger-Lift }\end{array}$ & 382 & 1,313 & 121 & 0.09 & 0.32 & 401 & 6,176 & 555 & 0.09 & 1.38 & 401 & 5,612 & 439 \\
\hline $\begin{array}{l}\text { Unconventional, } \\
\text { Non-Plunger }\end{array}$ & 89 & 189 & 570 & 3.02 & 6.41 & 501 & 19,392 & 20,005 & 1.02 & 39.93 & 501 & 19,370 & 18,245 \\
\hline $\begin{array}{l}\text { Unconventional, } \\
\text { Plunger-Lift }\end{array}$ & 4 & 8 & 1 & 0.13 & 0.27 & 27 & 377 & 59 & 0.15 & 2.22 & 27 & 374 & 38 \\
\hline Total & 1,269 & 5,330 & 5,911 & 1.11 & 4.66 & 1,801 & 59,660 & 32,863 & 0.54 & 18.24 & 1,801 & 59,086 & 30,219 \\
\hline
\end{tabular}

*Unallocated methane emissions from liquids unloading 
Table S40. Benchmarking liquids unloading activities in the Fort Worth Syncline basin, comparison between the 2016 GHGRP and NETL (this work)

\begin{tabular}{|c|c|c|c|c|c|c|c|c|c|c|c|c|c|}
\hline \multirow[b]{2}{*}{$\begin{array}{l}\text { Fort } \\
\text { Syncline }\end{array}$} & \multirow[b]{2}{*}{$\begin{array}{l}\text { Venting } \\
\text { Wells (\#) }\end{array}$} & \multicolumn{4}{|c|}{2016 GHGRP } & \multicolumn{5}{|c|}{ NETL (Stochastic) } & \multicolumn{3}{|c|}{ NETL (Deterministic) } \\
\hline & & $\begin{array}{l}\text { Venting } \\
\text { Events } \\
\text { (\#) }\end{array}$ & $\begin{array}{l}\text { *Methane } \\
\text { Emissions } \\
\left(\mathrm{MT} \mathrm{CH}_{4}\right)\end{array}$ & $\begin{array}{l}\text { *Methane } \\
\text { Emissions Per } \\
\text { Venting Event } \\
\text { (MT CH} / \text { event) }\end{array}$ & $\begin{array}{l}\text { *Methane } \\
\text { Emissions Per } \\
\text { Venting Well (MT } \\
\mathrm{CH}_{4} / \text { venting-well) }\end{array}$ & $\begin{array}{l}\text { Venting } \\
\text { Wells (\#) }\end{array}$ & $\begin{array}{l}\text { Venting } \\
\text { Events } \\
\text { (\#) }\end{array}$ & $\begin{array}{l}* \text { Methane } \\
\text { Emissions } \\
\left(\mathrm{MT} \mathrm{CH}_{4}\right)\end{array}$ & $\begin{array}{l}\text { *Methane } \\
\text { Emissions Per } \\
\text { Venting Event } \\
\text { (MT CH } \text { CHevent }_{4}\end{array}$ & $\begin{array}{l}\text { *Methane } \\
\text { Emissions Per } \\
\text { Venting Well (MT } \\
\mathrm{CH}_{4} / \text { venting-well) }\end{array}$ & $\begin{array}{l}\text { Venting } \\
\text { Wells (\#) }\end{array}$ & $\begin{array}{l}\text { Venting } \\
\text { Events (\#) }\end{array}$ & $\begin{array}{l}\text { *Methane } \\
\text { Emissions } \\
\left(\mathrm{MT} \mathrm{CH}_{4}\right)\end{array}$ \\
\hline $\begin{array}{l}\text { Conventional, } \\
\text { Non-Plunger }\end{array}$ & 20 & 43 & 5 & 0.11 & 0.24 & 69 & 2,670 & 225 & 0.08 & 3.26 & 69 & 2,669 & 204 \\
\hline $\begin{array}{l}\text { Conventional, } \\
\text { Plunger-Lift }\end{array}$ & 41 & 164 & 5 & 0.03 & 0.11 & 145 & 2,154 & 101 & 0.05 & 0.69 & 145 & 2,028 & 83 \\
\hline $\begin{array}{l}\text { Unconventional, } \\
\text { Non-Plunger }\end{array}$ & 362 & 808 & 170 & 0.21 & 0.47 & 248 & 9,564 & 3,580 & 0.38 & 14.42 & 248 & 9,597 & 3,195 \\
\hline $\begin{array}{l}\text { Unconventional, } \\
\text { Plunger-Lift }\end{array}$ & 492 & 1,428 & 193 & 0.14 & 0.39 & 352 & 5,118 & 409 & 0.08 & 1.16 & 352 & 4,931 & 239 \\
\hline Total & 915 & 2,443 & 372 & 0.15 & 0.41 & 815 & 19,506 & 4,315 & 0.20 & 5.30 & 815 & 19,225 & 3,720 \\
\hline
\end{tabular}

*Unallocated methane emissions from liquids unloading

Table S41. Benchmarking liquids unloading activities in the Green River basin, comparison between the 2016 GHGRP and NETL (this work)

\begin{tabular}{|c|c|c|c|c|c|c|c|c|c|c|c|c|c|}
\hline \multirow[b]{2}{*}{$\begin{array}{l}\text { Green } \\
\text { Basin }\end{array}$} & \multicolumn{5}{|c|}{2016 GHGRP } & \multicolumn{5}{|c|}{ NETL (Stochastic) } & \multicolumn{3}{|c|}{ NETL (Deterministic) } \\
\hline & $\begin{array}{l}\text { Venting } \\
\text { Wells (\#) }\end{array}$ & $\begin{array}{l}\text { Venting } \\
\text { Events } \\
\text { (\#) }\end{array}$ & $\begin{array}{l}\text { *Methane } \\
\text { Emissions } \\
\left(\mathrm{MT} \mathrm{CH}_{4}\right)\end{array}$ & $\begin{array}{l}\text { *Methane } \\
\text { Emissions Per } \\
\text { Venting Event } \\
\text { (MT CH} / \text { event) }\end{array}$ & $\begin{array}{l}\text { *Methane Emissions } \\
\text { Per Venting Well } \\
\text { (MT CH} / \text { venting- } \\
\text { well) }\end{array}$ & $\begin{array}{l}\text { Venting } \\
\text { Wells (\#) }\end{array}$ & $\begin{array}{l}\text { Venting } \\
\text { Events } \\
\text { (\#) }\end{array}$ & $\begin{array}{l}\text { *Methane } \\
\text { Emissions } \\
\left(\mathrm{MT} \mathrm{CH}_{4}\right)\end{array}$ & $\begin{array}{l}\text { *Methane } \\
\text { Emissions Per } \\
\text { Venting Event } \\
\text { (MT CH} 4 \text { /event) }\end{array}$ & $\begin{array}{l}\text { *Methane Emissions } \\
\text { Per Venting Well } \\
\text { (MT CH} / \text { venting- } \\
\text { well) }\end{array}$ & $\begin{array}{l}\text { Venting } \\
\text { Wells } \\
\text { (\#) }\end{array}$ & $\begin{array}{l}\text { Venting } \\
\text { Events } \\
(\#)\end{array}$ & $\begin{array}{l}\text { *Methane } \\
\text { Emissions } \\
\left(\mathrm{MT} \mathrm{CH}_{4}\right)\end{array}$ \\
\hline $\begin{array}{l}\text { Conventional, } \\
\text { Non-Plunger }\end{array}$ & 851 & 6,174 & 1,670 & 0.27 & 1.96 & 412 & 15,850 & 3,201 & 0.21 & 7.78 & 412 & 15,915 & 2,809 \\
\hline $\begin{array}{l}\text { Conventional, } \\
\text { Plunger-Lift }\end{array}$ & 1,875 & 15,636 & 1,588 & 0.10 & 0.85 & 658 & 16,491 & 1,747 & 0.11 & 2.65 & 658 & 11,007 & 1,001 \\
\hline $\begin{array}{l}\text { Unconventional, } \\
\text { Non-Plunger }\end{array}$ & 3 & 6 & 4 & 0.71 & 1.43 & 438 & 16,897 & 6,928 & 0.39 & 15.80 & 438 & 16,947 & 6,208 \\
\hline $\begin{array}{l}\text { Unconventional, } \\
\text { Plunger-Lift }\end{array}$ & - & - & - & - & - & - & - & - & - & - & - & & - \\
\hline Total & 2,729 & 21,816 & 3,262 & 0.15 & 1.20 & 1,508 & 49,237 & 11,877 & 0.23 & 7.88 & 1,508 & 43,869 & 10,018 \\
\hline
\end{tabular}

*Unallocated methane emissions from liquids unloading 
Table S42. Benchmarking liquids unloading activities in the Gulf Coast basin, comparison between the 2016 GHGRP and NETL (this work)

\begin{tabular}{|c|c|c|c|c|c|c|c|c|c|c|c|c|c|}
\hline & \multicolumn{5}{|c|}{2016 GHGRP } & \multicolumn{5}{|c|}{ NETL (Stochastic) } & \multicolumn{3}{|c|}{ NETL (Deterministic) } \\
\hline Gulf Coast Basin & $\begin{array}{l}\text { Venting } \\
\text { Wells (\#) }\end{array}$ & $\begin{array}{l}\text { Venting } \\
\text { Events } \\
\text { (\#) }\end{array}$ & $\begin{array}{l}\text { *Methane } \\
\text { Emissions } \\
\left(\mathrm{MT} \mathrm{CH}_{4}\right)\end{array}$ & $\begin{array}{l}\text { *Methane } \\
\text { Emissions Per } \\
\text { Venting Event } \\
(\mathrm{MT} \mathrm{CH} / \text { event) }\end{array}$ & $\begin{array}{l}\text { *Methane Emissions } \\
\text { Per Venting Well } \\
\text { (MT CH} / \text { venting- } \\
\text { well) }\end{array}$ & $\begin{array}{l}\text { Venting } \\
\text { Wells (\#) }\end{array}$ & $\begin{array}{l}\text { Venting } \\
\text { Events } \\
(\#)\end{array}$ & $\begin{array}{l}* \text { Methane } \\
\text { Emissions } \\
\left(\mathrm{MT} \mathrm{CH}_{4}\right)\end{array}$ & $\begin{array}{l}\text { *Methane } \\
\text { Emissions Per } \\
\text { Venting Event } \\
\text { (MT CH} 4 / \text { event) }\end{array}$ & $\begin{array}{l}\text { *Methane Emissions } \\
\text { Per Venting Well } \\
\text { (MT CH} / \text { venting- } \\
\text { well) }\end{array}$ & $\begin{array}{l}\text { Venting } \\
\text { Wells } \\
\text { (\#) }\end{array}$ & $\begin{array}{l}\text { Venting } \\
\text { Events } \\
(\#)\end{array}$ & $\begin{array}{l}\text { *Methane } \\
\text { Emissions } \\
\left(\mathrm{MT} \mathrm{CH}_{4}\right)\end{array}$ \\
\hline $\begin{array}{l}\text { Conventional, } \\
\text { Non-Plunger }\end{array}$ & 1,105 & 2,858 & 1,361 & 0.48 & 1.23 & 1,180 & 45,501 & 10,070 & 0.25 & 8.53 & 1,180 & 45,619 & 9,411 \\
\hline $\begin{array}{l}\text { Conventional, } \\
\text { Plunger-Lift }\end{array}$ & 88 & 871 & 136 & 0.16 & 1.55 & 69 & 1,129 & 133 & 0.12 & 1.94 & 69 & 965 & 100 \\
\hline $\begin{array}{l}\text { Unconventional, } \\
\text { Non-Plunger }\end{array}$ & 195 & 725 & 278 & 0.38 & 1.42 & 362 & 13,981 & 11,200 & 0.79 & 30.97 & 362 & 13,983 & 10,089 \\
\hline $\begin{array}{l}\text { Unconventional, } \\
\text { Plunger-Lift }\end{array}$ & 54 & 54 & 10 & 0.18 & 0.18 & 162 & 2,266 & 508 & 0.21 & 3.14 & 162 & 2,263 & 311 \\
\hline Total & 1,442 & 4,508 & 1,785 & 0.40 & 1.24 & 1,772 & 62,878 & 21,912 & 0.40 & 12.36 & 1,772 & 62,829 & 19,912 \\
\hline
\end{tabular}

*Unallocated methane emissions from liquids unloading

Table S43. Benchmarking liquids unloading activities in the Permian basin, comparison between the 2016 GHGRP and NETL (this work)

\begin{tabular}{|c|c|c|c|c|c|c|c|c|c|c|c|c|c|}
\hline & \multicolumn{5}{|c|}{2016 GHGRP } & \multicolumn{5}{|c|}{ NETL (Stochastic) } & \multicolumn{3}{|c|}{ NETL (Deterministic) } \\
\hline Permian Basin & $\begin{array}{l}\text { Venting } \\
\text { Wells (\#) }\end{array}$ & $\begin{array}{l}\text { Venting } \\
\text { Events (\#) }\end{array}$ & $\begin{array}{l}\text { *Methane } \\
\text { Emissions } \\
\left(\mathrm{MT} \mathrm{CH}_{4}\right)\end{array}$ & $\begin{array}{l}\text { *Methane } \\
\text { Emissions Per } \\
\text { Venting Event (MT } \\
\mathrm{CH}_{4} / \text { event) }\end{array}$ & $\begin{array}{l}\text { *Methane } \\
\text { Emissions Per } \\
\text { Venting Well } \\
\text { (MT } \\
\mathrm{CH}_{4} / \text { venting- } \\
\text { well) }\end{array}$ & $\begin{array}{l}\text { Venting } \\
\text { Wells (\#) }\end{array}$ & $\begin{array}{l}\text { Venting } \\
\text { Events (\#) }\end{array}$ & $\begin{array}{l}\text { *Methane } \\
\text { Emissions } \\
\left(\mathrm{MT} \mathrm{CH}_{4}\right)\end{array}$ & $\begin{array}{l}\text { *Methane } \\
\text { Emissions Per } \\
\text { Venting Event (MT } \\
\mathrm{CH}_{4} / \text { event) }\end{array}$ & $\begin{array}{l}\text { *Methane } \\
\text { Emissions Per } \\
\text { Venting Well } \\
\text { (MT } \\
\mathrm{CH}_{4} / \text { venting- } \\
\text { well) }\end{array}$ & $\begin{array}{l}\text { Venting } \\
\text { Wells (\#) }\end{array}$ & $\begin{array}{l}\text { Venting } \\
\text { Events (\#) }\end{array}$ & $\begin{array}{l}\text { *Methane } \\
\text { Emissions } \\
\left(\mathrm{MT} \mathrm{CH}_{4}\right)\end{array}$ \\
\hline $\begin{array}{l}\text { Conventional, Non- } \\
\text { Plunger }\end{array}$ & 194 & 320 & 108 & 0.34 & 0.56 & 102 & 3,965 & 432 & 0.12 & 4.23 & 102 & 3,953 & 411 \\
\hline $\begin{array}{l}\text { Conventional, } \\
\text { Plunger-Lift }\end{array}$ & 184 & 381 & 90 & 0.24 & 0.49 & 177 & 2,516 & 175 & 0.08 & 0.99 & 177 & 2,477 & 161 \\
\hline $\begin{array}{l}\text { Unconventional, } \\
\text { Non-Plunger }\end{array}$ & 26 & 49 & 12 & 0.25 & 0.48 & 67 & 2,607 & 1,043 & 0.40 & 15.50 & 67 & 2,602 & 910 \\
\hline $\begin{array}{l}\text { Unconventional, } \\
\text { Plunger-Lift }\end{array}$ & - & - & - & - & - & - & - & - & - & - & - & - & - \\
\hline Total & 404 & 750 & 211 & 0.28 & 0.52 & 347 & 9,9088 & 1,650 & 0.19 & 4.76 & 347 & 9,032 & 1,482 \\
\hline
\end{tabular}

*Unallocated methane emissions from liquids unloading 
Table S44. Benchmarking liquids unloading activities in the Piceance basin, comparison between the 2016 GHGRP and NETL (this work)

\begin{tabular}{|c|c|c|c|c|c|c|c|c|c|c|c|c|c|}
\hline & \multicolumn{5}{|c|}{2016 GHGRP } & \multicolumn{5}{|c|}{ NETL (Stochastic) } & \multicolumn{3}{|c|}{ NETL (Deterministic) } \\
\hline Piceance Basin & $\begin{array}{l}\text { Venting } \\
\text { Wells (\#) }\end{array}$ & $\begin{array}{l}\text { Venting } \\
\text { Events } \\
\text { (\#) }\end{array}$ & $\begin{array}{l}\text { *Methane } \\
\text { Emissions } \\
\left(\mathrm{MT} \mathrm{CH}_{4}\right)\end{array}$ & $\begin{array}{l}\text { *Methane } \\
\text { Emissions Per } \\
\text { Venting Event } \\
\text { (MT CH} \mathrm{CH}_{4} / \text { event) }\end{array}$ & $\begin{array}{l}\text { *Methane } \\
\text { Emissions Per } \\
\text { Venting Well (MT } \\
\mathrm{CH}_{4} / \text { venting-well) }\end{array}$ & $\begin{array}{l}\text { Venting } \\
\text { Wells (\#) }\end{array}$ & $\begin{array}{l}\text { Venting } \\
\text { Events } \\
\text { (\#) }\end{array}$ & $\begin{array}{l}\text { *Methane } \\
\text { Emissions } \\
\left(\mathrm{MT} \mathrm{CH}_{4}\right)\end{array}$ & $\begin{array}{l}\text { *Methane } \\
\text { Emissions Per } \\
\text { Venting Event } \\
\text { (MT } \mathrm{CH}_{4} / \text { event) }\end{array}$ & $\begin{array}{l}\text { *Methane } \\
\text { Emissions Per } \\
\text { Venting Well (MT } \\
\mathrm{CH}_{4} / \text { venting-well) }\end{array}$ & $\begin{array}{l}\text { Venting } \\
\text { Wells (\#) }\end{array}$ & $\begin{array}{l}\text { Venting } \\
\text { Events } \\
\text { (\#) }\end{array}$ & $\begin{array}{l}\text { *Methane } \\
\text { Emissions } \\
\left(\mathrm{MT} \mathrm{CH}_{4}\right)\end{array}$ \\
\hline $\begin{array}{l}\text { Conventional, Non- } \\
\text { Plunger }\end{array}$ & 343 & 8,757 & 341 & 0.04 & 1.00 & 41 & 1,598 & 199 & 0.12 & 4.82 & 41 & 1,600 & 186 \\
\hline $\begin{array}{l}\text { Conventional, } \\
\text { Plunger-Lift }\end{array}$ & 3,744 & 23,465 & 1,398 & 0.06 & 0.37 & 455 & 8,483 & 451 & 0.05 & 0.99 & 455 & 6,362 & 321 \\
\hline $\begin{array}{l}\text { Unconventional, } \\
\text { Non-Plunger }\end{array}$ & 52 & 69 & 14 & 0.21 & 0.28 & 1,318 & 50,803 & 17,199 & 0.35 & 13.05 & 1,318 & 50,964 & 16,708 \\
\hline $\begin{array}{l}\text { Unconventional, } \\
\text { Plunger-Lift }\end{array}$ & 3 & 25 & 10 & 0.41 & 3.45 & 374 & 6,112 & 426 & 0.07 & 1.14 & 374 & 5,238 & 330 \\
\hline Total & 4,142 & 32,316 & 1,765 & 0.05 & 0.43 & 2,189 & 66,996 & 18,275 & 0.22 & 8.35 & 2,189 & 64,165 & 17,545 \\
\hline
\end{tabular}

*Unallocated methane emissions from liquids unloading

Table S45. Benchmarking liquids unloading activities in the San Juan basin, comparison between the 2016 GHGRP and NETL (this work)

\begin{tabular}{|c|c|c|c|c|c|c|c|c|c|c|c|c|c|}
\hline & \multicolumn{5}{|c|}{2016 GHGRP } & \multicolumn{5}{|c|}{ NETL (Stochastic) } & \multicolumn{3}{|c|}{ NETL (Deterministic) } \\
\hline San Juan Basin & $\begin{array}{l}\text { Venting } \\
\text { Wells (\#) }\end{array}$ & $\begin{array}{l}\text { Venting } \\
\text { Events } \\
\text { (\#) }\end{array}$ & $\begin{array}{l}\text { *Methane } \\
\text { Emissions } \\
\left(\mathrm{MT} \mathrm{CH}_{4}\right)\end{array}$ & $\begin{array}{l}\text { *Methane } \\
\text { Emissions Per } \\
\text { Venting Event } \\
\text { (MT CH} / \text { event) }\end{array}$ & $\begin{array}{l}\text { Methane } \\
\text { Emissions Per } \\
\text { Venting Well (MT } \\
\mathrm{CH}_{4} / \text { venting-well) }\end{array}$ & $\begin{array}{l}\text { Venting } \\
\text { Wells (\#) }\end{array}$ & $\begin{array}{l}\text { Venting } \\
\text { Events } \\
\text { (\#) }\end{array}$ & $\begin{array}{l}\text { *Methane } \\
\text { Emissions } \\
\left(\mathrm{MT} \mathrm{CH}_{4}\right)\end{array}$ & $\begin{array}{l}\text { *Methane } \\
\text { Emissions Per } \\
\text { Venting Event } \\
(\mathrm{MT} \mathrm{CH} / \text { event) }\end{array}$ & $\begin{array}{l}\text { *Methane } \\
\text { Emissions Per } \\
\text { Venting Well (MT } \\
\mathrm{CH}_{4} / \text { venting-well) }\end{array}$ & $\begin{array}{l}\text { Venting } \\
\text { Wells (\#) }\end{array}$ & $\begin{array}{l}\text { Venting } \\
\text { Events } \\
(\#)\end{array}$ & $\begin{array}{l}\text { *Methane } \\
\text { Emissions } \\
\left.\text { ( } \mathrm{MT} \mathrm{CH}_{4}\right)\end{array}$ \\
\hline $\begin{array}{l}\text { Conventional, Non- } \\
\text { Plunger }\end{array}$ & 728 & 9,170 & 707 & 0.08 & 0.97 & 994 & 39,103 & 4,251 & 0.11 & 4.27 & 994 & 38,439 & 3,681 \\
\hline $\begin{array}{l}\text { Conventional, } \\
\text { Plunger-Lift }\end{array}$ & 4,349 & 795,021 & 16,967 & 0.02 & 3.90 & 5,995 & $2,439,245$ & 63,506 & 0.03 & 10.59 & 5,995 & $1,092,801$ & 27,821 \\
\hline $\begin{array}{l}\text { Unconventional, } \\
\text { Non-Plunger }\end{array}$ & 161 & 989 & 175 & 0.18 & 1.09 & 222 & 8,672 & 3,333 & 0.38 & 15.02 & 222 & 8,581 & 2,991 \\
\hline $\begin{array}{l}\text { Unconventional, } \\
\text { Plunger-Lift }\end{array}$ & 206 & 24,107 & 320 & 0.01 & 1.55 & 297 & 60,465 & 2,102 & 0.04 & 7.08 & 297 & 34,797 & 1,114 \\
\hline Total & $5, \mathbf{4 4 4}$ & 829,287 & 18,169 & 0.02 & 3.34 & 7,508 & $2,547,485$ & 73,191 & 0.03 & 9.75 & 7,508 & $1,174,617$ & 35,607 \\
\hline
\end{tabular}

*Unallocated methane emissions from liquids unloading 
Table S46. Benchmarking liquids unloading activities in the South Oklahoma Folded Belt basin, comparison between the 2016 GHGRP and NETL (this work)

\begin{tabular}{|c|c|c|c|c|c|c|c|c|c|c|c|c|c|}
\hline \multirow[b]{2}{*}{$\begin{array}{l}\text { South Oklahoma } \\
\text { Folded Belt }\end{array}$} & \multicolumn{5}{|c|}{2016 GHGRP } & \multicolumn{5}{|c|}{ NETL (Stochastic) } & \multicolumn{3}{|c|}{ NETL (Deterministic) } \\
\hline & $\begin{array}{l}\text { Venting } \\
\text { Wells (\#) }\end{array}$ & $\begin{array}{l}\text { Venting } \\
\text { Events } \\
\text { (\#) }\end{array}$ & $\begin{array}{l}\text { *Methane } \\
\text { Emissions } \\
\left(\mathrm{MT} \mathrm{CH}_{4}\right)\end{array}$ & $\begin{array}{l}\text { Methane } \\
\text { Emissions Per } \\
\text { Venting Event } \\
\text { (MT CH } \text { CHevent) }_{4} \text {. }\end{array}$ & $\begin{array}{l}\text { *Methane Emissions } \\
\text { Per Venting Well } \\
\text { (MT CH} / \text { venting- } \\
\text { well) }\end{array}$ & $\begin{array}{l}\text { Venting } \\
\text { Wells (\#) }\end{array}$ & $\begin{array}{l}\text { Venting } \\
\text { Events } \\
\text { (\#) }\end{array}$ & $\begin{array}{l}* \text { Methane } \\
\text { Emissions } \\
\left(\mathrm{MT} \mathrm{CH}_{4}\right)\end{array}$ & $\begin{array}{l}\text { Methane } \\
\text { Emissions Per } \\
\text { Venting Event } \\
\text { (MT CH} / \text { event) }\end{array}$ & $\begin{array}{l}\text { *Methane Emissions } \\
\text { Per Venting Well } \\
\text { (MT CH} / \text { venting- } \\
\text { well) }\end{array}$ & $\begin{array}{l}\text { Venting } \\
\text { Wells } \\
\text { (\#) }\end{array}$ & $\begin{array}{l}\text { Venting } \\
\text { Events } \\
\text { (\#) }\end{array}$ & $\begin{array}{l}\text { *Methane } \\
\text { Emissions } \\
\left(\mathrm{MT} \mathrm{CH}_{4}\right)\end{array}$ \\
\hline $\begin{array}{l}\text { Conventional, } \\
\text { Non-Plunger }\end{array}$ & 3 & 6 & 12 & 1.93 & 3.86 & 4 & 170 & 17 & 0.10 & 3.90 & 4 & 274,514 & 16 \\
\hline $\begin{array}{l}\text { Conventional, } \\
\text { Plunger-Lift }\end{array}$ & 41 & 147 & 9 & 0.06 & 0.21 & 50 & 741 & 49 & 0.07 & 0.97 & 50 & 31,004 & 42 \\
\hline $\begin{array}{l}\text { Unconventional, } \\
\text { Non-Plunger }\end{array}$ & 14 & 16 & 49 & 3.04 & 3.48 & 15 & 596 & 376 & 0.59 & 24.38 & 15 & 540 & 317 \\
\hline $\begin{array}{l}\text { Unconventional, } \\
\text { Plunger-Lift }\end{array}$ & 19 & 124 & 39 & 0.31 & 2.04 & 20 & 317 & 31 & 0.10 & 1.57 & 20 & 15,746 & 17 \\
\hline Total & 77 & 293 & 108 & 0.37 & 1.40 & 90 & 1,824 & 473 & 0.24 & 5.27 & 90 & 321,805 & 392 \\
\hline
\end{tabular}

*Unallocated methane emissions from liquids unloading

Table S47. Benchmarking liquids unloading activities in the Strawn basin, comparison between the 2016 GHGRP and NETL (this work)

\begin{tabular}{|c|c|c|c|c|c|c|c|c|c|c|c|c|c|}
\hline \multirow[b]{2}{*}{ Strawn Basin } & \multicolumn{5}{|c|}{2016 GHGRP } & \multicolumn{5}{|c|}{ NETL (Stochastic) } & \multicolumn{3}{|c|}{ NETL (Deterministic) } \\
\hline & $\begin{array}{l}\text { Venting } \\
\text { Wells (\#) }\end{array}$ & $\begin{array}{l}\text { Venting } \\
\text { Events } \\
\text { (\#) }\end{array}$ & $\begin{array}{l}* \text { Methane } \\
\text { Emissions } \\
\left(\mathrm{MT} \mathrm{CH}_{4}\right)\end{array}$ & $\begin{array}{l}\text { *Methane } \\
\text { Emissions Per } \\
\text { Venting Event } \\
\text { (MT CH } 4 \text { /event) }\end{array}$ & $\begin{array}{l}\text { *Methane } \\
\text { Emissions Per } \\
\text { Venting Well (MT } \\
\mathrm{CH}_{4} / \text { venting-well) }\end{array}$ & $\begin{array}{l}\text { Venting } \\
\text { Wells (\#) }\end{array}$ & $\begin{array}{l}\text { Venting } \\
\text { Events } \\
\text { (\#) }\end{array}$ & $\begin{array}{l}\text { *Methane } \\
\text { Emissions } \\
\left(\mathrm{MT} \mathrm{CH}_{4}\right)\end{array}$ & $\begin{array}{l}\text { *Methane } \\
\text { Emissions Per } \\
\text { Venting Event } \\
\text { (MT CH} 4 / \text { event) }\end{array}$ & $\begin{array}{l}\text { *Methane } \\
\text { Emissions Per } \\
\text { Venting Well (MT } \\
\mathrm{CH}_{4} / \text { venting-well) }\end{array}$ & $\begin{array}{l}\text { Venting } \\
\text { Wells (\#) }\end{array}$ & $\begin{array}{l}\text { Venting } \\
\text { Events } \\
\text { (\#) }\end{array}$ & $\begin{array}{l}\text { *Methane } \\
\text { Emissions } \\
\left(\mathrm{MT} \mathrm{CH}_{4}\right)\end{array}$ \\
\hline $\begin{array}{l}\text { Conventional, } \\
\text { Non-Plunger }\end{array}$ & 13 & 29 & 1 & 0.05 & 0.11 & 14 & 523 & 44 & 0.07 & 3.22 & 14 & 524 & 39 \\
\hline $\begin{array}{l}\text { Conventional, } \\
\text { Plunger-Lift }\end{array}$ & 9 & 22 & 0 & 0.01 & 0.03 & 9 & 134 & 5 & 0.04 & 0.54 & 9 & 131 & 4 \\
\hline $\begin{array}{l}\text { Unconventional, } \\
\text { Non-Plunger }\end{array}$ & 356 & 844 & 456 & 0.54 & 1.28 & 441 & 17,009 & 6,813 & 0.38 & 15.47 & 441 & 17,030 & 5,812 \\
\hline $\begin{array}{l}\text { Unconventional, } \\
\text { Plunger-Lift }\end{array}$ & 866 & 3,049 & 1,015 & 0.33 & 1.17 & 1,025 & 15,751 & 1,328 & 0.09 & 1.30 & 1,025 & 14,341 & 750 \\
\hline Total & 1,244 & 3,944 & 1,473 & 0.37 & 1.18 & 1,488 & 33,417 & 8,189 & 0.22 & 5.50 & 1,488 & 32,025 & 6,604 \\
\hline
\end{tabular}

*Unallocated methane emissions from liquids unloading 
Table S48. Benchmarking liquids unloading activities in the Uinta basin, comparison between the 2016 GHGRP and NETL (this work)

\begin{tabular}{|c|c|c|c|c|c|c|c|c|c|c|c|c|c|}
\hline & \multicolumn{5}{|c|}{2016 GHGRP } & \multicolumn{5}{|c|}{ NETL (Stochastic) } & \multicolumn{3}{|c|}{ NETL (Deterministic) } \\
\hline Uinta Basin & $\begin{array}{l}\text { Venting } \\
\text { Wells (\#) }\end{array}$ & $\begin{array}{l}\text { Venting } \\
\text { Events } \\
\text { (\#) }\end{array}$ & $\begin{array}{l}\text { *Methane } \\
\text { Emissions } \\
\left(\mathrm{MT} \mathrm{CH}_{4}\right)\end{array}$ & $\begin{array}{l}\text { *Methane } \\
\text { Emissions Per } \\
\text { Venting Event } \\
(\mathrm{MT} \mathrm{CH} / \text { event) }\end{array}$ & $\begin{array}{l}\text { *Methane } \\
\text { Emissions Per } \\
\text { Venting Well (MT } \\
\mathrm{CH}_{4} / \text { venting-well) }\end{array}$ & $\begin{array}{l}\text { Venting } \\
\text { Wells (\#) }\end{array}$ & $\begin{array}{l}\text { Venting } \\
\text { Events } \\
\text { (\#) }\end{array}$ & $\begin{array}{l}\text { *Methane } \\
\text { Emissions } \\
\left(\mathrm{MT} \mathrm{CH}_{4}\right)\end{array}$ & $\begin{array}{l}\text { *Methane } \\
\text { Emissions Per } \\
\text { Venting Event } \\
\text { (MT CH} / \text { event) }\end{array}$ & $\begin{array}{l}\text { *Methane } \\
\text { Emissions Per } \\
\text { Venting Well (MT } \\
\mathrm{CH}_{4} / \text { venting-well) }\end{array}$ & $\begin{array}{l}\text { Venting } \\
\text { Wells (\#) }\end{array}$ & $\begin{array}{l}\text { Venting } \\
\text { Events } \\
\text { (\#) }\end{array}$ & $\begin{array}{l}\text { *Methane } \\
\text { Emissions } \\
\left(\mathrm{MT} \mathrm{CH}_{4}\right)\end{array}$ \\
\hline $\begin{array}{l}\text { Conventional, } \\
\text { Non-Plunger }\end{array}$ & 345 & 1,564 & 467 & 0.30 & 1.35 & 171 & 6,584 & 1,312 & 0.20 & 7.67 & 171 & 6,615 & 1,206 \\
\hline $\begin{array}{l}\text { Conventional, } \\
\text { Plunger-Lift }\end{array}$ & 977 & 43,758 & 3,506 & 0.08 & 3.59 & 630 & 43,812 & 2,533 & 0.06 & 4.02 & 630 & 21,452 & 1,188 \\
\hline $\begin{array}{l}\text { Unconventional, } \\
\text { Non-Plunger }\end{array}$ & - & - & - & - & - & - & - & - & - & - & - & - & - \\
\hline $\begin{array}{l}\text { Unconventional, } \\
\text { Plunger-Lift }\end{array}$ & - & - & - & - & - & - & - & - & - & - & - & - & - \\
\hline Total & 1,322 & 45,322 & 3,973 & 0.09 & 3.01 & 802 & 50,396 & 3,844 & 0.08 & 4.80 & 802 & 28,067 & 2,395 \\
\hline
\end{tabular}

*Unallocated methane emissions from liquids unloading

Table S49. Benchmarking liquids unloading activities in the Williston basin, comparison between the 2016 GHGRP and NETL (this work)

\begin{tabular}{|c|c|c|c|c|c|c|c|c|c|c|c|c|c|}
\hline & \multicolumn{5}{|c|}{2016 GHGRP } & \multicolumn{5}{|c|}{ NETL (Stochastic) } & \multicolumn{3}{|c|}{ NETL (Deterministic) } \\
\hline Williston Basin & $\begin{array}{l}\text { Venting } \\
\text { Wells (\#) }\end{array}$ & $\begin{array}{l}\text { Venting } \\
\text { Events } \\
(\#)\end{array}$ & $\begin{array}{l}\text { *Methane } \\
\text { Emissions } \\
\left(\mathrm{MT} \mathrm{CH}_{4}\right)\end{array}$ & $\begin{array}{l}\text { *Methane } \\
\text { Emissions Per } \\
\text { Venting Event } \\
\text { (MT CH} / \text { event) }\end{array}$ & $\begin{array}{l}\text { *Methane } \\
\text { Emissions Per } \\
\text { Venting Well (MT } \\
\mathrm{CH}_{4} / \text { venting-well) }\end{array}$ & $\begin{array}{l}\text { Venting } \\
\text { Wells (\#) }\end{array}$ & $\begin{array}{l}\text { Venting } \\
\text { Events } \\
(\#)\end{array}$ & $\begin{array}{l}\text { *Methane } \\
\text { Emissions } \\
\left(\mathrm{MT} \mathrm{CH}_{4}\right)\end{array}$ & $\begin{array}{l}\text { *Methane } \\
\text { Emissions Per } \\
\text { Venting Event } \\
\text { (MT CH} / \text { event) }\end{array}$ & $\begin{array}{l}\text { *Methane } \\
\text { Emissions Per } \\
\text { Venting Well (MT } \\
\mathrm{CH}_{4} / \text { venting-well) }\end{array}$ & $\begin{array}{l}\text { Venting } \\
\text { Wells (\#) }\end{array}$ & $\begin{array}{l}\text { Venting } \\
\text { Events } \\
\text { (\#) }\end{array}$ & $\begin{array}{l}\text { *Methane } \\
\text { Emissions } \\
\left(\mathrm{MT} \mathrm{CH}_{4}\right)\end{array}$ \\
\hline $\begin{array}{l}\text { Conventional, } \\
\text { Non-Plunger }\end{array}$ & 435 & 371,397 & 323 & 0.00 & 0.74 & 477 & 18,748 & 185 & 0.01 & 0.39 & 477 & 18,423 & 133 \\
\hline $\begin{array}{l}\text { Conventional, } \\
\text { Plunger-Lift }\end{array}$ & 6 & 86 & 5 & 0.06 & 0.88 & 0 & 6 & 0 & 0.05 & 1.16 & 0 & 4 & 0 \\
\hline $\begin{array}{l}\text { Unconventional, } \\
\text { Non-Plunger }\end{array}$ & - & - & - & - & - & - & - & - & - & - & - & - & - \\
\hline $\begin{array}{l}\text { Unconventional, } \\
\text { Plunger-Lift }\end{array}$ & - & - & - & - & - & - & - & - & - & - & - & - & - \\
\hline Total & 441 & 371,483 & 328 & 0.00 & 0.74 & 477 & 18,754 & 185 & 0.01 & 0.39 & 477 & 18,427 & 133 \\
\hline
\end{tabular}

*Unallocated methane emissions from liquids unloading 


\section{S13. References}

1. U.S. Environmental Protection Agency, Inventory of U.S. Greenhouse Gas Emissions and Sinks: 1990-2016. In EPA, Ed. 2017.

2. Terri Shires; Miriam Lev-On, Characterizing Pivotal Sources of Methane Emissions from Natural Gas Production: Summary and Analysis of API and ANGA Survey Responses. Final report, Washington, DC 2012.

3. Allen, D. T.; Sullivan, D. W.; Zavala-Araiza, D.; Pacsi, A. P.; Harrison, M.; Keen, K.; Fraser, M. P.; Daniel Hill, A.; Lamb, B. K.; Sawyer, R. F.; Seinfeld, J. H., Methane Emissions from Process Equipment at Natural Gas Production Sites in the United States: Liquid Unloadings. Environmental Science \& Technology 2015, 49, (1), 641-648.

4. $\quad$ DrillingInfo DrillingInfo Desktop Application, 2018.

5. E.W. Lemmon; M.O. McLinden; D.G. Friend, Thermophysical Properties of Fluid Systems. In NIST Chemistry WebBook, NIST Standard Reference Database Number 69, P.J. Linstrom; W.G. Mallard, Eds. National Institute of Standards and Technology: Gaithersburg MD, 2018; Vol. 20899.

6. EIA, Annual Energy Outlook 2016. Table G.1. Heat Contents. In Energy Information Administration., 2016; Vol. 2018.

7. Zavala-Araiza, D.; Allen, D. T.; Harrison, M.; George, F. C.; Jersey, G. R., Allocating Methane Emissions to Natural Gas and Oil Production from Shale Formations. ACS Sustainable Chemistry \& Engineering 2015, 3, (3), 492-498. 